\title{
The Lyman Alpha Reference Sample
}

\section{Efficient turbulence-driven Ly $\alpha$ escape and an analysis of IR, CO, and [C II]158 $\mu \mathrm{m}^{\star, \star \star}$}

\author{
J. Puschnig ${ }^{1,2}$, M. Hayes ${ }^{1}$, G. Östlin ${ }^{1}$, J. Cannon ${ }^{3}$, I. Smirnova-Pinchukova ${ }^{4}$, B. Husemann ${ }^{4}$, D. Kunth ${ }^{5}$, J. Bridge ${ }^{6}$, \\ E. C. Herenz ${ }^{7}$, M. Messa ${ }^{8}$, and I. Oteo ${ }^{9,10}$
}

1 Department of Astronomy, University of Stockholm, AlbaNova, Stockholm, Sweden e-mail: johannes@sonnensystem.at

2 Argelander-Institut für Astronomie, Auf dem Hügel 71, 53121 Bonn, Germany e-mail: johannes.puschnig@uni-bonn.de

3 Department of Physics \& Astronomy, Macalester College, 1600 Grand Avenue, Saint Paul, MN 55105, USA

${ }^{4}$ Max Planck Institute for Astronomy, Königstuhl 17, 69117 Heidelberg, Germany

5 Institut d'Astrophysique de Paris (IAP), 98bis Boulevard Arago, 75014 Paris, France

${ }^{6}$ University of Louisville, Natural Science Bldg. 208, Louisville, KY 40292, USA

7 European Southern Observatory, Av. Alonso de Córdova 3107, 7630355 Vitacura, Santiago, Chile

8 Department of Astronomy, University of Massachusetts-Amherst, Amherst, MA 01003, USA

9 Institute for Astronomy, University of Edinburgh, Royal Observatory, Blackford Hill, Edinburgh EH9 3HJ, UK

10 European Southern Observatory, Karl-Schwarzschild-Str. 2, 85748 Garching, Germany

Received 23 September 2019 / Accepted 21 April 2020

\begin{abstract}
Context. Lyman- $\alpha(\operatorname{Ly} \alpha)$ is the brightest emission line in star-forming galaxies. However, its interpretation in terms of physical properties is hampered by the resonant nature of Ly $\alpha$ photons. In order to remedy this complicated situation, the Lyman Alpha Reference Sample (LARS) was defined, enabling the study of Ly $\alpha$ production and escape mechanisms in 14 local star-forming galaxies.

Aims. With this paper, we complement our efforts and study the global dust and (molecular) gas content as well as the properties of gas associated with photon-dominated regions. We aim to characterize the interstellar medium of LARS galaxies, allowing us to relate these newly derived properties to quantities relevant for Ly $\alpha$ escape.

Methods. We observed LARS galaxies with Herschel, SOFIA, the IRAM 30m telescope, and APEX, targeting far-infrared (FIR) continuum and emission lines of [C II] $158 \mu \mathrm{m}$, [O I] $63 \mu \mathrm{m}$, [O III] $88 \mu \mathrm{m}$, and low- $J$ CO lines. Using Bayesian methods we derived dust model parameters and estimated the total gas masses for all LARS galaxies, taking into account a metallicity-dependent gas-todust ratio. Star formation rates were estimated from FIR, [C II] $158 \mu \mathrm{m}$, and [O I] $63 \mu \mathrm{m}$ luminosities.

Results. LARS covers a wide dynamic range in the derived properties, with FIR-based star formation rates from $\sim 0.5-100 M_{\odot} \mathrm{yr}^{-1}$, gas fractions between $\sim 15-80 \%$, and gas depletion times ranging from a few hundred megayears up to more than ten gigayears. The distribution of LARS galaxies in the $\Sigma_{\text {gas }}$ versus $\Sigma_{\text {SFR }}$ (Kennicutt-Schmidt plane) is thus quite heterogeneous. However, we find that LARS galaxies with the longest gas depletion times, that is, relatively high gas surface densities $\left(\Sigma_{\text {gas }}\right)$ and low star formation rate densities $\left(\Sigma_{\mathrm{SFR}}\right)$, have by far the highest Ly $\alpha$ escape fraction. A strong approximately linear relation is found between the Ly $\alpha$ escape fraction and the total gas $\left(\mathrm{HI}+\mathrm{H}_{2}\right)$ depletion time. We argue that the $\mathrm{Ly} \alpha$ escape in those galaxies is driven by turbulence in the star-forming gas that shifts the $\operatorname{Ly} \alpha$ photons out of resonance close to the places where they originate. We further report on an extreme [C II] $158 \mu \mathrm{m}$ excess in LARS 5, corresponding to $\sim 14 \pm 3 \%$ of the FIR luminosity, which probably is the most extreme [C II]-to-FIR ratio observed in a galaxy (without active nucleus) to date.
\end{abstract}

Key words. galaxies: evolution - galaxies: starburst - galaxies: irregular - galaxies: ISM - infrared: galaxies - radio lines: galaxies

\footnotetext{
* The reduced spectra are only available at the CDS via anonymous ftp to cdsarc.u-strasbg.fr (130.79.128.5) or via http://cdsarc . u-strasbg.fr/viz-bin/cat/J/A+A/644/A10

$\star \star$ This work is based on observations with Herschel, an ESA space observatory with science instruments provided by European-led Principal Investigator consortia and with important participation from NASA. It is also based on observations made with the NASA/DLR Stratospheric Observatory for Infrared Astronomy (SOFIA). SOFIA is jointly operated by the Universities Space Research Association, Inc. (USRA), under NASA contract NNA17BF53C, and the Deutsches SOFIA Institut (DSI) under DLR contract 50 OK 0901 to the University of Stuttgart. It is also based on observations carried out with the IRAM 30m Telescope. IRAM is supported by INSU/CNRS (France), MPG (Germany), and IGN (Spain). This publication is based on data acquired with the Atacama Pathfinder Experiment (APEX). APEX is a collaboration between the MaxPlanck-Institut für Radioastronomie, the European Southern Observatory, and the Onsala Space Observatory. This work is also based on observations made with the NASA/ESA Hubble Space Telescope, obtained at the Space Telescope Science Institute, which is operated by the Association of Universities for Research in Astronomy, Inc., under NASA contract NAS 5-26555. These observations are associated with programme GO 12310.
} 


\section{Introduction}

More than fifty years ago, Partridge \& Peebles (1967) advocated the search for galaxies at high redshifts using a very narrow spectral window around the (redshifted) Lyman alpha (Ly $\alpha$ ) hydrogen emission line at $1216 \AA$ rest wavelength. According to their prediction, the bulk of the ionizing photons in young galaxies are ultimately converted to a single line, Ly $\alpha$, making it the brightest spectral feature, and that which holds a substantial fraction of the bolometric flux. In practice, however, it turned out to be very difficult to find $\operatorname{Ly} \alpha$ emitters and it was not until 1981, when Meier \& Terlevich (1981) discovered weak Ly $\alpha$ emission in a local blue compact galaxy. It took another 17 years until Cowie \& $\mathrm{Hu}$ (1998) found a substantial population of $\operatorname{Ly} \alpha$ emitters at $z \sim 3.4$. Although the number of high- $z$ galaxies detected through the Ly $\alpha$ line has increased ever since (Ouchi et al. 2008, 2010; Adams et al. 2011; Matthee et al. 2015; Santos et al. 2016; Herenz et al. 2017; Sobral et al. 2018; Urrutia et al. 2019), the decades-long endeavour made it evident that some physical processes are at play that may suppress or enhance Ly $\alpha$ emission.

Nevertheless, it is known that resonant scattering of Ly $\alpha$ photons leads to a complex radiative transport problem (Neufeld 1990; Ahn et al. 2001, 2002; Dijkstra et al. 2006a,b; Verhamme et al. 2006, 2008; Schaerer \& Verhamme 2008). The line strength and visibility of Ly $\alpha$ depends on many factors such as dust content (Hayes et al. 2010), dust geometry (Scarlata et al. 2009), neutral gas content (Zheng et al. 2017), kinematics (Kunth et al. 1998; Cannon et al. 2004; Wofford et al. 2013; Pardy et al. 2014), and gas geometry (Tenorio-Tagle et al. 1999; Mas-Hesse et al. 2003; Duval et al. 2014; Jaskot \& Oey 2014). Hayes (2015) offer a review of the issue. While, for example, large scale galactic winds may enhance Ly $\alpha$ escape via a high velocity gradient along which the photons are shifted to wavelengths less prone to further scattering, dust works in the other direction. Since the resonant nature of the line drastically increases the path length out of the galaxy, the chance for Ly $\alpha$ photons to be destroyed by dust is significantly enhanced. Remarkably, Ly $\alpha$ photons do not appear to diffuse very far in space, because their re-distribution scales are small (Bridge et al. 2018), meaning that Ly $\alpha$ photons get stuck locally over many scattering events. As a result, in some galaxies the interstellar medium is optically thin even for UV photons (e.g. at a hydrogen column density of $10^{16} \mathrm{~cm}^{-2}$ ), but opaque for Ly $\alpha$ (Verhamme et al. 2015; Puschnig et al. 2017).

Given the fact that the total escape fraction of Ly $\alpha$ photons depends on such a large variety of effects that all act together in a complex way, predictions of $\operatorname{Ly} \alpha$ emission are difficult. However, progress has been made recently (Trainor et al. 2019; Sobral \& Matthee 2019). In particular, Runnholm et al. (2020) reproduced the Ly $\alpha$ output of local Ly $\alpha$ emitters with a dispersion of $\sim 0.3$ dex only.

One way to remedy this complicated situation is to study $\operatorname{Ly} \alpha$ production and escape mechanisms in a sample of star-forming galaxies on a spatially resolved basis. To do so, LARS - the Lyman Alpha Reference Sample - was defined (Hayes et al. 2013, 2014; Östlin et al. 2014). It is a sample of 14 galaxies (see Table 1 for basic properties), which is statistically meaningful enough to observe trends (i.e. it covers a wide range in stellar mass, star formation rate, and metallicity) and, most importantly, its selection of galaxies are comparable to galaxies observed at high- $z$ (Ouchi et al. 2008; Cowie et al. 2011). LARS galaxies are selected according to two main parameters: UV luminosity and $\mathrm{H} \alpha$ equivalent width. The former is comparable to Lyman Break galaxies at redshifts $\sim 3-4$, and the latter is $>100 \AA$, limiting the sample to galaxies with currently ongoing (young) bursts of star formation. Multi-band imaging with the Hubble Space Telescope (HST) forms the groundwork of our study, which includes HST ultraviolet spectroscopy (Rivera-Thorsen et al. 2015), optical broadband imaging (Micheva et al. 2018), integral-field spectroscopy with the Potsdam Multi-Aperture Spectrophotometer (Herenz et al. 2016), Green Bank Telescope $21 \mathrm{~cm}$ spectra (Pardy et al. 2014), and JVLA data (Le Reste et al., in prep.).

The Kennicutt-Schmidt relation or KS law (Schmidt 1959; Kennicutt 1998) is key for studies of star formation, because it relates gas surface densities to star formation rate (SFR) surface densities. In its classical form, total gas $\left(\mathrm{HI}+\mathrm{H}_{2}\right)$ versus SFR, the slope is typically found to be non-linear with power-law indices of $\sim 1.4-1.5$ (Kennicutt 1998). In contrast, the molecular gas relation is often found to be linear (e.g. Wong \& Blitz 2002; Bigiel et al. 2008, 2011; Leroy et al. 2008, 2013; Bolatto et al. 2017; Utomo et al. 2018; Colombo et al. 2018), and it holds over eight orders of magnitude in mass, on a wide range of spatial scales - from tens of parsec (Wu et al. 2005) to entire galaxies - and even in the low-density disc outskirts of galaxies (Schruba et al. 2011) as well as in low-metallicity dwarf galaxies (Bolatto et al. 2011; Jameson et al. 2016). The observed linear slope has far reaching consequences: (1) the timescale over which the gas is consumed at the current SFR, $M_{\text {gas }} / \mathrm{SFR}$, or the gas depletion time, is constant and (2) the driving physics behind the KS law is not self-gravity alone (Semenov et al. 2019), and other processes (e.g. feedback) must play a fundamental role as well. However, non-linear molecular KS relations are also observed, namely on even smaller scales (e.g. Evans et al. 2009, 2014; Heiderman et al. 2010; Gutermuth et al. 2011), in starburst galaxies (e.g. Genzel et al. 2010, 2015), in the dwarf magellanic irregular galaxy NGC 4449 (Calzetti et al. 2018), and also in galactic centres (e.g. Leroy et al. 2013).

Gao \& Solomon (2004a,b) and Lada et al. (2010) further show that star formation linearly scales with the dense gas traced by $\mathrm{HCN}(1-0)$. However, it is still a matter of debate which of these scaling relations (molecular or dense gas) is the more fundamental. Lada et al. (2012) offer a discussion on the issue. In recent years, however, great efforts have been undertaken to study the dense gas in external galaxies (Bigiel et al. 2016; Jiménez-Donaire et al. 2019), showing that the environment and feedback play a fundamental role (Kruijssen et al. 2019).

Observations of galaxies at redshifts between 0 and 4 have shown that the level of star formation is mainly dictated by stellar mass, and regulated by secular processes (Popesso et al. 2019). This is seen as a tight relation between stellar mass and SFR, the so called main sequence of star forming galaxies (Brinchmann et al. 2004; Noeske et al. 2007; Daddi et al. 2007; Elbaz et al. 2007; Peng et al. 2010; Wuyts et al. 2011; Whitaker et al. 2012, 2014; Tomczak et al. 2016). While the slope of the relation does not vary with redshift, its intercept shifts towards higher SFRs (see for example Wuyts et al. 2011; Rodighiero et al. 2011). However, for main sequence galaxies it was also found that even out to redshift $z \sim 4$ galaxies have constant gas depletion times due to an increase in the gas fraction (Tacconi et al. 2013; Genzel et al. 2015; Scoville et al. 2017). It was also realised that the consumption timescales are too short compared to the age of the Universe. Thus, galaxies must accrete gas, for example from the circumgalactic medium or from other galaxies through mergers, in order to sustain star formation. 
Table 1. Compilation of properties of LARS galaxies relevant for this work.

\begin{tabular}{|c|c|c|c|c|c|c|c|c|c|c|c|}
\hline ID & $z$ & $\begin{array}{c}\log _{10} M_{*} \\
{\left[M_{\odot}\right]} \\
\end{array}$ & $\begin{array}{c}Z \\
{[12+\log \mathrm{O} / \mathrm{H}]} \\
\end{array}$ & $\begin{array}{c}\log _{10} M_{\mathrm{HI}} \\
{\left[M_{\odot}\right]} \\
\end{array}$ & $\begin{array}{c}L_{\mathrm{LyA}} \\
{\left[10^{42} \mathrm{cgs}\right]}\end{array}$ & $f_{\mathrm{esc}}^{\mathrm{LyA}}$ & $\begin{array}{l}D_{\text {scatt }} \\
{[\mathrm{kpc}]} \\
\end{array}$ & $\begin{array}{c}D_{25_{\text {SDSS }}} \\
{\left[{ }^{\prime \prime}\right]} \\
\end{array}$ & $b / a_{\mathrm{SDSS}}$ & $\begin{array}{c}R_{\mathrm{cut}_{\mathrm{M} 18}} \\
{[\mathrm{kpc}]} \\
\end{array}$ & $b / a_{\mathrm{M} 18}$ \\
\hline 1 & 0.02782 & 9.785 & 8.24 & 9.3979 & 0.85 & 0.119 & 0.72 & 14.94 & 0.69 & 2.69 & 0.71 \\
\hline 2 & 0.02982 & 9.371 & 8.23 & 9.4472 & 0.81 & 0.521 & 0.36 & 25.67 & 0.43 & 2.00 & 0.39 \\
\hline 3 & 0.03143 & 10.303 & 8.41 & 9.8388 & 0.10 & 0.003 & 0.35 & 85.80 & 0.22 & 2.34 & 0.29 \\
\hline 4 & 0.03249 & 10.111 & 8.19 & 9.8865 & 0.00 & 0.000 & 0.70 & 19.07 & 0.89 & 4.21 & 0.64 \\
\hline 5 & 0.03375 & 9.630 & 8.12 & $<9.4624$ & 1.11 & 0.174 & 0.40 & 13.11 & 0.73 & 2.20 & 0.71 \\
\hline 6 & 0.03461 & 9.320 & 8.08 & ${ }^{(*)}<10.3617$ & 0.00 & 0.000 & 0.41 & 20.32 & 0.60 & 2.05 & 0.55 \\
\hline 7 & 0.03774 & 9.677 & 8.35 & 9.4914 & 1.01 & 0.100 & 0.37 & 19.03 & 0.48 & 2.33 & 0.55 \\
\hline 8 & 0.03825 & 10.970 & 8.51 & 10.3424 & 1.00 & 0.025 & 0.44 & 30.84 & 0.59 & 5.10 & 1.00 \\
\hline 9 & 0.04677 & 10.708 & 8.37 & 10.1139 & 0.33 & 0.007 & 0.56 & 7.37 & 0.86 & 6.70 & 0.50 \\
\hline 10 & 0.05765 & 10.332 & 8.51 & 9.6532 & 0.16 & 0.026 & 0.52 & 22.44 & 0.43 & 3.12 & 0.60 \\
\hline 11 & 0.08461 & 11.083 & 8.44 & 10.415 & 1.20 & 0.036 & 1.56 & 33.52 & 0.28 & 8.40 & 0.24 \\
\hline 12 & 0.10210 & 9.870 & 8.34 & - & 0.93 & 0.009 & 1.03 & 8.71 & 0.81 & 4.44 & 0.88 \\
\hline 13 & 0.14670 & 10.772 & 8.50 & - & 0.72 & 0.010 & 2.24 & 10.26 & 0.59 & 7.40 & 0.71 \\
\hline 14 & 0.18070 & 9.243 & 8.06 & - & 4.46 & 0.163 & 1.68 & 5.46 & 0.82 & 5.03 & 1.00 \\
\hline
\end{tabular}

Notes. The redshifts were derived from radial velocities (given in the optical definition) in Pardy et al. (2014) and are thus related to the H I systemic velocities for all LARS galaxies except 12-14 (which have no H I detection). For those, Pardy et al. (2014) report optical Sloan Digital Sky Survey (SDSS) velocities. Stellar masses $\left(\log _{10} M_{*}\right)$, Ly $\alpha$ luminosities $\left(L_{\mathrm{LyA}}\right)$, and escape fractions $\left(f_{\mathrm{esc}}^{\mathrm{LyA}}\right)$ were previously published by Hayes et al. (2014). The metallicities ( $Z$ ) derived through the empirical O3N2 relation are taken from Östlin et al. (2014), and atomic gas masses $\left(\log _{10} M_{\mathrm{HI}}\right)$ from Pardy et al. (2014). ${ }^{(*)}$ We note that the atomic mass reported for LARS 6 is contaminated by the field spiral UGC10028 and thus is only an upper limit. Typical Ly $\alpha$ scattering distances $\left(D_{\text {scatt }}\right)$ for the galaxies were derived by Bridge et al. (2018) and the radii at which the star formation rate density has decreased to $0.01 M_{\odot} \mathrm{yr}^{-1} \mathrm{kpc}^{2}\left(R_{\mathrm{cut}_{\mathrm{M} 18}}\right)$, together with axis ratios $\left(b / a_{\mathrm{M} 18}\right)$ were calculated by Micheva et al. (2018). The blue-band diameters of the $25 \mathrm{mag} \operatorname{arcsec}^{2}$ isophote $\left(D_{25_{\text {SDSS }}}\right)$ as well as $b / a_{\text {SDSS }}$ were derived from SDSS $g$-band observations using an SQL query on SDSS DR7. Since the SDSS $g$-band isophote typically gives sizes that are $\sim 1.3$ times larger than those measured in the Johnson $B$ band (Hakobyan et al. 2012), we divided the $g$-band diameters by that factor and report estimated values for $D_{25_{\text {SDss }}}$ here.

In this paper, we establish the KS relation, calculate gas fractions, gas depletion times, and star formation efficiencies in LARS. This allows us to study the dependencies between previously derived quantities related to Ly $\alpha$ emission and the global properties of star-forming gas, giving new insights into physical processes and environmental properties that efficiently drive the escape of Ly $\alpha$ photons. We performed low-J CO spectroscopy with the $30 \mathrm{~m}$ telescope of the Institut de radioastronomie millimétrique (IRAM), far-infrared imaging and spectroscopy with Herschel/PACS and SOFIA/FIFI-LS, targeting continuum and fine structure emission lines of [C II] $158 \mu \mathrm{m}$ and [O I] $63 \mu \mathrm{m}$.

We used the aforementioned radio and far infrared observations (see a description of the observations in Sect. 2) to derive the properties of the interstellar medium (ISM) from dust models (Sect. 3.1) and to establish the KS law for LARS galaxies and study the features of the environment: molecular and total gas fractions, star formation rates and efficiencies, and gas depletion times (Sect. 4.2). In addition we used far-infrared photometry and spectroscopy to explore photon-dominated regions (PDR) within LARS (Sect. 4.5), that is, the far ultraviolet (FUV) radiation field and densities. In Sect. 5 we finally discuss which of the derived properties influence Ly $\alpha$ escape and put our results into the context of the early phases of disc formation. Throughout the paper we adopt a cosmology with $H_{0}=70, \Omega_{\mathrm{M}}=0.3$, and $\Omega_{\mathrm{vac}}=0.7$.

\section{Observations and archival data}

In this section, the observing strategy, instrumental setup, and data reduction are described. We further present spectra, flux measurements, and upper limits for our observations and the archival data used in the study.

\subsection{Observations of molecular lines of $\mathrm{CO}, \mathrm{HCN}$, and $\mathrm{HCO}^{+}$}

Observations with the IRAM $30 \mathrm{~m}$ telescope were carried out under programmes 082-14, 064-15, and 178-15. Data were acquired during the heterodyne pool weeks in September, October, and November 2014 as well as in January and February 2016. Observing runs in visitor mode were carried out in June and September 2015, as well as during May 2016. In total, more than $110 \mathrm{~h}$ of observing time were scheduled at the telescope, from which roughly $1 / 3$ was lost due to poor weather conditions. Using SFR and metallicity as indicators for the chance of detecting $\mathrm{CO}$, we first selected ten galaxies out of our sample for molecular line observations; we initially rejected LARS 4, 5, 6, and 14 as targets. Finally, we included LARS 5, given the fact that the galaxy has strong winds (Duval et al. 2016) that might trigger molecular gas formation.

Five galaxies out of our sample were detected (simultaneously) in $\mathrm{CO}(1-0)$ and $\mathrm{CO}(2-1)$ using the Eight Mixer Receiver (EMIR). The observed CO lines are shown in Fig. 1 on the main beam brightness temperature scale. Beam sizes, main beam efficiencies, Gaussian fit results, and flux densities per beam are summarized in Table 2. We note that we also detected $\mathrm{CO}(1-$ 0 ) in the companion galaxy of LARS 11. As it is not part of our sample, no analysis was performed. Six LARS galaxies remained undetected down to a baseline rms level of $\sim 1 \mathrm{mK}$ at $20 \mathrm{~km} \mathrm{~s}^{-1}$, corresponding to a flux density of roughly $5 \mathrm{mJy}$ per $\mathrm{CO}(1-0)$ beam (see Table 3 and Fig. 2). Additionally, in the two galaxies with the strongest CO emission, LARS 3 and 8, HCN and $\mathrm{HCO}^{+}(1-0)$ were detected, however at a signal-to-noise ratio of 3-6 only (see Fig. 3).

Additional $\mathrm{CO}(3-2)$ observations of two galaxies were carried out with APEX, the Atacama Pathfinder Experiment (Güsten et al. 2006) within the Swedish time allocation during 

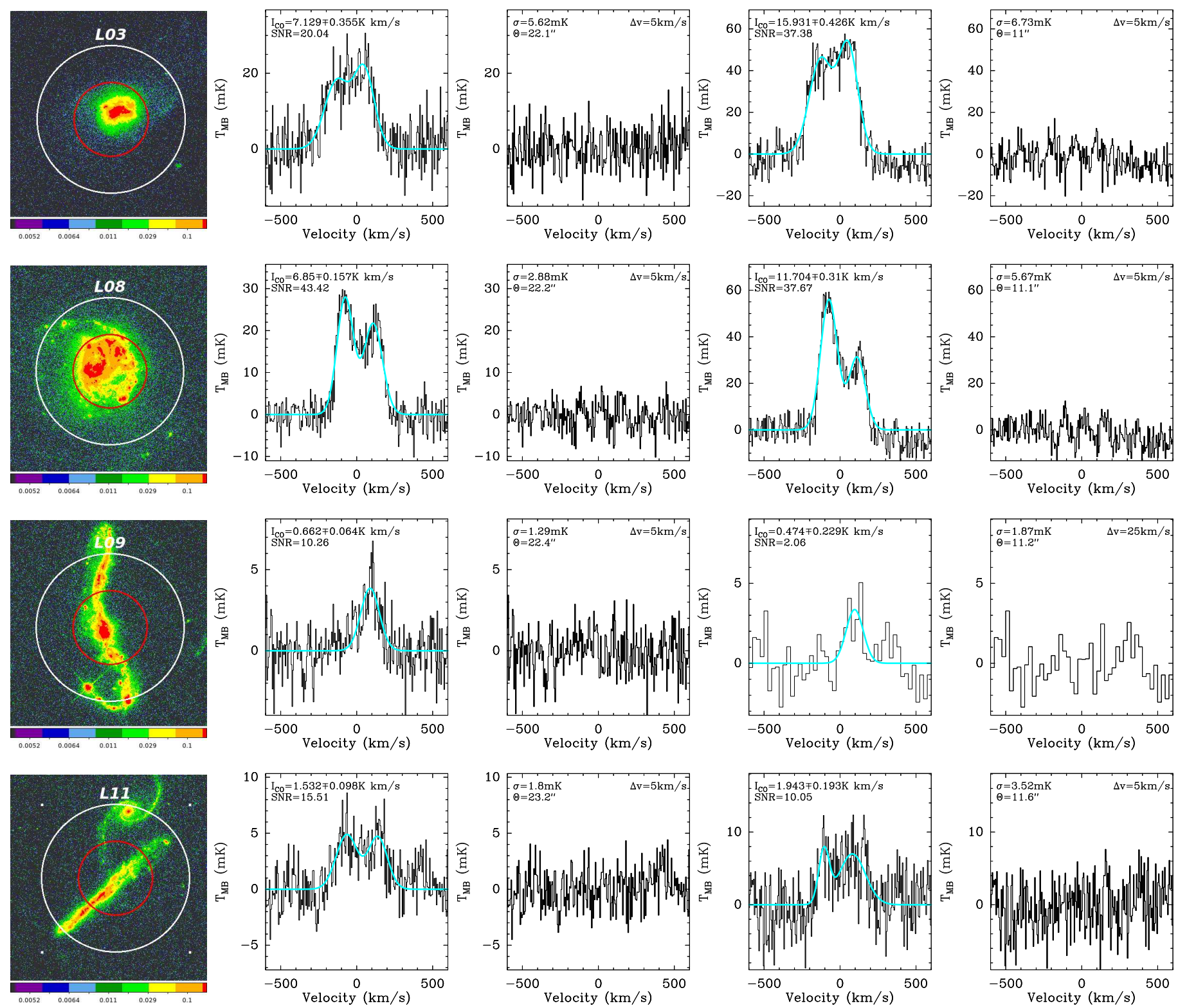

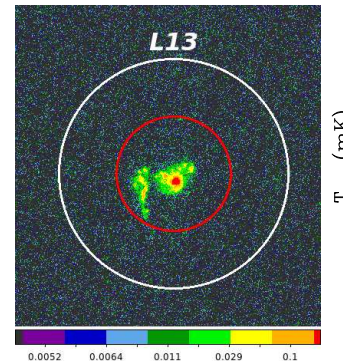

(1) $\mathrm{H} \alpha$ \& HPBW

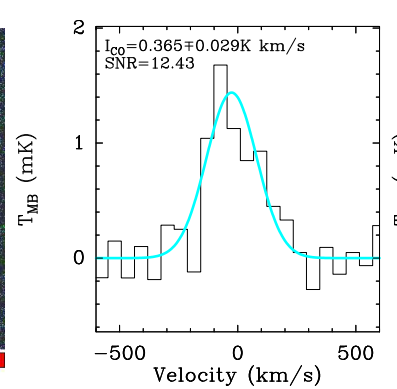

(2) $\mathrm{CO}(1-0)$

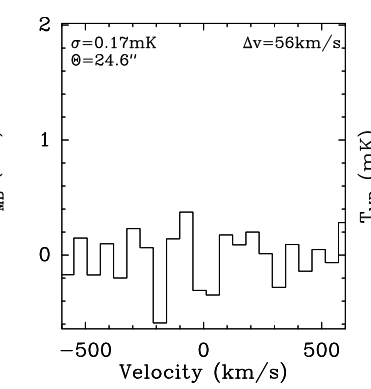

(3) residual

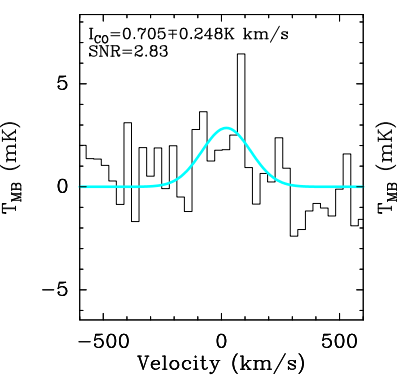

(4) $\mathrm{CO}(2-1)$

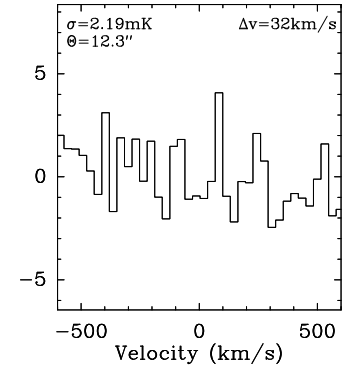

(5) residual

Fig. 1. $\mathrm{CO}(1-0)$ and (2-1) emission lines of LARS galaxies obtained with the IRAM 30m telescope, shown on the $T_{\mathrm{MB}}$ scale in milli Kelvin. The beam sizes for the transitions are shown in Col. (1) on top of our HST H $\alpha$ narrow band images: white: $\mathrm{CO}(1-0)$, and red: $\mathrm{CO}(2-1)$. $\mathrm{CO}(1-0)$ and $\mathrm{CO}(2-1)$ spectra are shown in Cols. (2) and (4) respectively. Single or double Gaussian fits were performed to calculate the line flux. They are shown as cyan solid lines. The residuals after subtraction of the fits from the data are given in Cols. (3) and (5).

periods P94 and P96 under project codes F-9340A-2014 and F9329A-2015. LARS 8 was detected in $\mathrm{CO}(2-1)$ and (3-2) and LARS 13 was detected in $\mathrm{CO}(3-2)$ using the Swedish heterodyne Facility Instrument (Belitsky et al. 2006; Vassilev et al. 2008).
Alls molecular scans (IRAM 30m and APEX) were obtained in wobbler-switching mode and the calibrated data were reduced using GILDAS/CLASS ${ }^{1}$, a collection of state-of-the-art software

1 http://wWW.iram.fr/IRAMFR/GILDAS 
Table 2. Summary of the detected molecular lines.

\begin{tabular}{|c|c|c|c|c|c|c|c|c|c|}
\hline ID & Line & $B_{\text {eff }}$ & $\begin{array}{c}\theta \\
{[\operatorname{arcsec}]}\end{array}$ & $\begin{array}{l}t_{\text {on }} \\
{[\mathrm{h}]}\end{array}$ & $\begin{array}{r}v_{\text {peak }} \\
{\left[\mathrm{km} \mathrm{s}^{-1}\right]}\end{array}$ & $\begin{array}{r}F W H M \\
{\left[\mathrm{~km} \mathrm{~s}^{-1}\right]}\end{array}$ & $S / N$ & $\begin{array}{r}I_{\mathrm{mb}} \\
{\left[\mathrm{K} \mathrm{km} \mathrm{s}^{-1}\right]} \\
\end{array}$ & $\begin{array}{r}S \\
{\left[\mathrm{Jy} \mathrm{km} \mathrm{s}^{-1}\right]} \\
\end{array}$ \\
\hline 3 & $\mathrm{CO}(1-0)$ & 0.79 & 22.10 & 0.6 & $49.6[17.6]-129.8[26.4]$ & 157.7 [29.35] 190.7 [41.43] & 20.0 & $7.13 \pm 0.36$ & $35.6 \pm 1.8$ \\
\hline 3 & $\mathrm{CO}(2-1)$ & 0.58 & 11.00 & 0.6 & -127.2 [10] 53.1 [7.9] & 178.3 [16.13] 152.9 [12.2] & 37.4 & $15.93 \pm 0.43$ & $79.6 \pm 2.1$ \\
\hline 3 & $\mathrm{HCN}(1-0)$ & 0.84 & 28.70 & 1.3 & $-40.3[51.5]$ & $623.7[126.79]$ & 7.0 & $1.06 \pm 0.15$ & $5.3 \pm 0.8$ \\
\hline 3 & $\mathrm{HCO}^{+}(1-0)$ & 0.84 & 28.60 & 1.3 & $-64.4[56.7]$ & $556.4[136.97]$ & 6.1 & $1.08 \pm 0.18$ & $5.4 \pm 0.9$ \\
\hline 8 & $\mathrm{CO}(1-0)$ & 0.80 & 22.20 & 3.2 & $-75.8[2.8] 108.4[3.9]$ & 127 [6.17] 144.7 [8.16] & 43.4 & $6.85 \pm 0.16$ & $34.2 \pm 0.8$ \\
\hline 8 & $\mathrm{CO}(2-1)$ & 0.59 & 11.10 & 3.4 & $-68[0.2] 118.9[2.6]$ & $139.1[4.04] 114.9[5.82]$ & 37.7 & $11.7 \pm 0.31$ & $58.5 \pm 1.6$ \\
\hline 8 & $\mathrm{CO}(3-2)$ & 0.73 & 18.50 & 0.9 & $-59.8[4.4] 124.6[4.7]$ & $148.7[10.45] 118.5[10.19]$ & 29.8 & $6.5 \pm 0.22$ & $203.1 \pm 6.8$ \\
\hline 8 & $\mathrm{HCN}(1-0)$ & 0.84 & 28.90 & 3.2 & $-121.9[26.5]$ & $151.8[47.73]$ & 4.0 & $0.29 \pm 0.07$ & $1.5 \pm 0.4$ \\
\hline 8 & $\mathrm{HCO}^{+}(1-0)$ & 0.84 & 28.70 & 3.2 & $-30.9[36.1] 0[0]$ & $216.4[98.46]$ & 3.2 & $0.19 \pm 0.06$ & $0.9 \pm 0.3$ \\
\hline 9 & $\mathrm{CO}(1-0)$ & 0.80 & 22.40 & 6.9 & $90[5.6]$ & $143.1[17.01]$ & 9.9 & $0.66 \pm 0.07$ & $3.3 \pm 0.3$ \\
\hline 9 & $\mathrm{CO}(2-1)$ & 0.59 & 11.20 & 4.5 & $97.1[19.5]$ & $130.9[40.19]$ & 3.0 & $0.51 \pm 0.17$ & $2.6 \pm 0.8$ \\
\hline 11 & $\mathrm{CO}(1-0)$ & 0.80 & 23.20 & 3.1 & $4.9[15.8] 139.1[14.8]$ & $165.6[29.89] 149.2[39.68]$ & 15.5 & $1.53 \pm 0.1$ & $7.7 \pm 0.5$ \\
\hline 11 & $\mathrm{CO}(2-1)$ & 0.60 & 11.60 & 1.4 & $-85[3.6] 101.8$ [9.7] & $123.6[16.35] 159.6[16.24]$ & 10.1 & $1.94 \pm 0.19$ & $9.7 \pm 1.0$ \\
\hline 13 & $\mathrm{CO}(1-0)$ & 0.82 & 24.60 & 4.0 & $-20.2[21.3]$ & $256.1[50.78]$ & 11.3 & $0.38 \pm 0.03$ & $1.9 \pm 0.2$ \\
\hline 13 & $\mathrm{CO}(2-1)$ & 0.63 & 12.30 & 3.3 & $42.6[33]$ & 242.9 [79.22] & 6.1 & $0.62 \pm 0.1$ & $3.1 \pm 0.5$ \\
\hline 13 & $\mathrm{CO}(3-2)$ & 0.73 & 20.50 & 5.4 & $-11[24.1]$ & 320.8 [65.29] & 6.2 & $0.52 \pm 0.08$ & $16.2 \pm 2.6$ \\
\hline
\end{tabular}

Notes. For each transition, we show the beam efficiency $B_{\text {eff }}$ that was used to translate the antenna temperature to the $T_{\mathrm{mb}}$ scale, the beam size $\theta$, the total effective on-source observing time (after processing and filtering) $T_{\text {on }}$, as well as results from Gaussian fits and fluxes. The velocity at the Gaussian peak brightness temperature, $v_{\text {peak }}$, is given with respect to the systemic velocity of HI and its error is shown in square brackets. In cases with more than one Gaussian fit, $v_{\text {peak }}$ is given for each component. FWHM is the full-width-at-half-maximum calculated from the Gaussian sigma value(s), and $\mathrm{S} / \mathrm{N}$ is the signal-to-noise ratio. The velocity-integrated source brightness temperatures on the main beam brightness temperature scale, as well as velocity-integrated flux densities, are indicated by $I_{\mathrm{mb}}$ and $S$ respectively.

oriented toward (sub-)millimetre radio astronomical applications. IRAM scans obtained with the Fast Fourier Transform Spectrometer (FTS) unit were corrected for platforming. In our pipeline, we initially chose a wide constant line window ranging from -300 to $+300 \mathrm{~km} \mathrm{~s}^{-1}$ around the expected zero-velocity position of the line, extracted a range of -1000 to $+1000 \mathrm{~km} \mathrm{~s}^{-1}$, and applied a linear baseline correction on each individual scan. In the next step, we masked spikes and then examined plots of system temperature and rms noise for all scans, which we used to filter scans that do not follow the radiometer equation. Scans that were obtained under conditions of high precipitable water vapour, when the atmospheric transmission at the given frequency was lower than $\sim 33 \%$, were excluded from further processing. The remaining scans per galaxy were average stacked and re-sampled to a resolution of $1 \mathrm{~km} \mathrm{~s}^{-1}$. From that point on, each galaxy was manually processed using individual line windows, baseline fits, and smoothing to resolutions between 5 and $56 \mathrm{~km} \mathrm{~s}^{-1}$ per channel. Gaussian fits were performed to reveal velocity components. Total fluxes were derived via the summation of channels along the line window. Errors on the fluxes are the rms multiplied by the number of channels along the window.

\subsection{Infrared observations}

In the following the spectra of the observed fine-structure lines ([C II $] 158 \mu \mathrm{m},[\mathrm{O}$ III $] 88 \mu \mathrm{m}$, [O I] $63 \mu \mathrm{m}$ ) are presented. The data reduction and subsequent derivation of the line fluxes are described for our spectra and also for the photometric data we took from the archive.

\subsubsection{Herschel/PACS observations}

Far infrared observations of LARS galaxies $(2,4,6,8,9)$ were executed with the Photoconductor Array Camera and Spec-
Table 3. Summary of LARS galaxies not detected in CO.

\begin{tabular}{lccccc}
\hline \hline ID & $B_{\text {eff }}$ & $\begin{array}{c}\mathrm{HPBW} \\
{[\operatorname{arcsec}]}\end{array}$ & $\begin{array}{c}t_{\text {on }} \\
{[\mathrm{h}]}\end{array}$ & $\begin{array}{c}\sigma_{\mathrm{rms}, 20 \mathrm{~km} \mathrm{~s}^{-1}} \\
{[\mathrm{mK}]}\end{array}$ & $\begin{array}{c}\sigma_{\mathrm{rms}, 20 \mathrm{~km} \mathrm{~s}^{-1}} \\
{[\mathrm{mJy}]}\end{array}$ \\
\hline 1 & 0.79 & 22.0 & 3.40 & 1.01 & 5.06 \\
2 & 0.79 & 22.0 & 1.90 & 1.38 & 6.93 \\
5 & 0.79 & 22.1 & 3.55 & 0.77 & 3.88 \\
7 & 0.80 & 22.2 & 5.70 & 0.68 & 3.42 \\
10 & 0.80 & 22.6 & 1.05 & 1.71 & 8.57 \\
12 & 0.81 & 23.6 & 2.30 & 0.90 & 4.54 \\
\hline
\end{tabular}

trometer (PACS; Poglitsch et al. 2010) on board the Herschel Space Observatory (Pilbratt et al. 2010) under programme OT2_mhayes_4, for a total time of $12.1 \mathrm{~h}$. Our observations were carried out in the standard single pointing chop-nod mode. PACS was operated in parallel mode (using the photometer and spectrometer simultaneously), targeting photometry at 70, 100, and $160 \mu \mathrm{m}$, as well as spectral lines of [O I] $63 \mu \mathrm{m},[\mathrm{O}$ III] $88 \mu \mathrm{m}$, and [C II] $158 \mu \mathrm{m}$. The point spread function (PSF) is well described by a two-dimensional Gaussian with sizes of roughly $6 \times 12$, $7 \times 13$, and $12 \times 16$ arcsec for the blue, green, and red channel respectively.

Further PACS imaging of LARS galaxies is found in the archive. LARS 3 was observed under the guarantee time key programme KPGT_esturm_1 (PI: Eckhard Sturm) as part of a comprehensive far-infrared survey of infrared bright galaxies. LARS 5 was observed in programme OT2_dhunter_4 (PI: Deidre Hunter), as part of an investigation of dust and star formation in local low-metallicity galaxies. LARS 7 and 10 are covered by programme KPOT_seales_01_2, the Herschel Thousand Degree Survey (PI: Stephen Eales). LARS 12, 13, and 14 were observed as part of a survey of local Lyman Break analogues under programme OT1_roverzie_1 (PI: Roderik Overzier). 

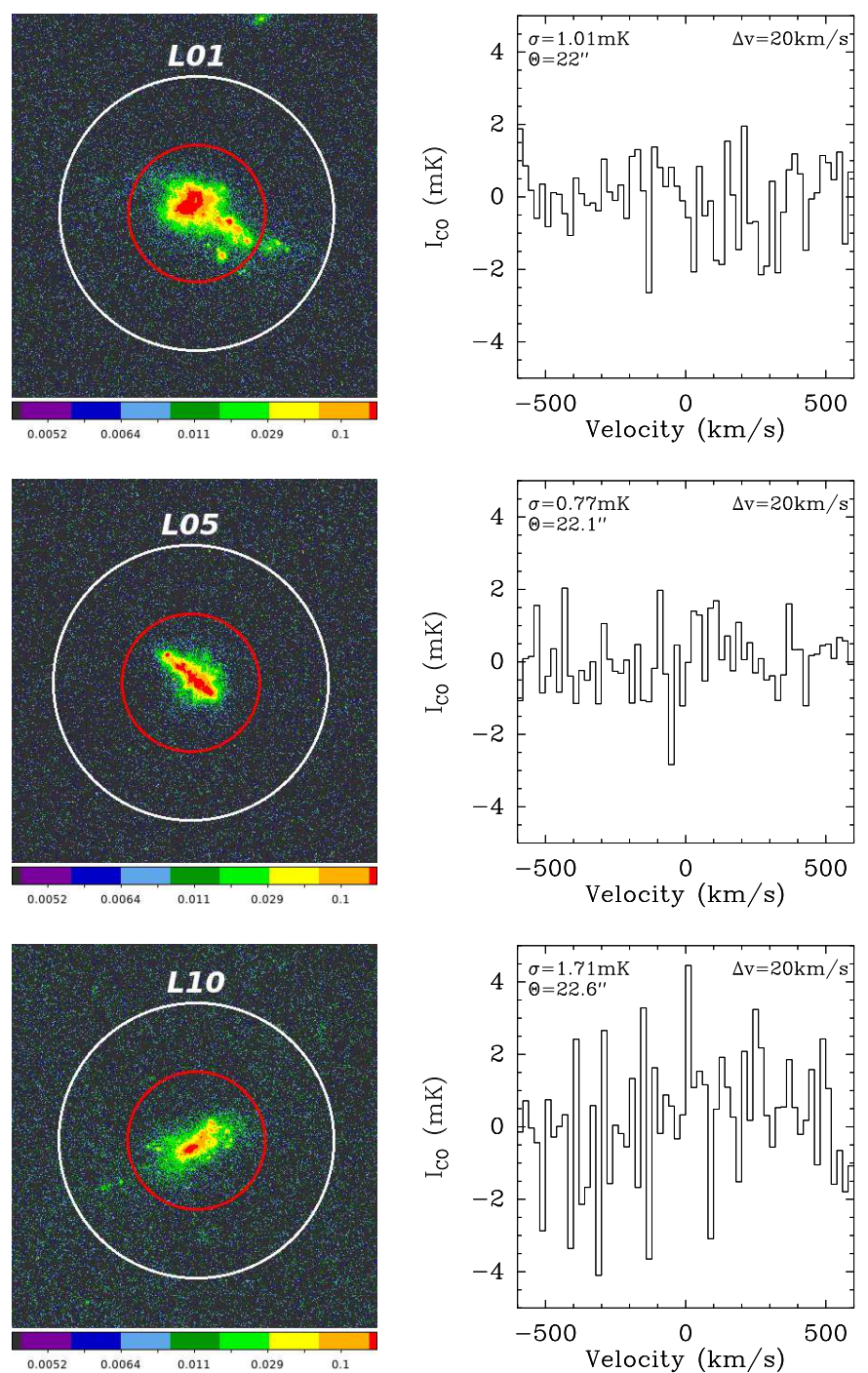

(1) $\mathrm{H} \alpha \&$ HPBW

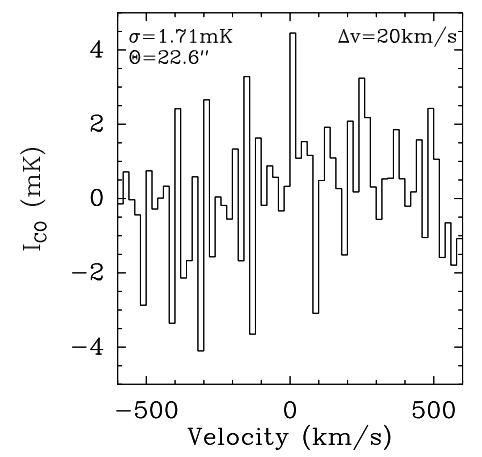

(2) $\mathrm{T}_{\mathrm{MB}}$
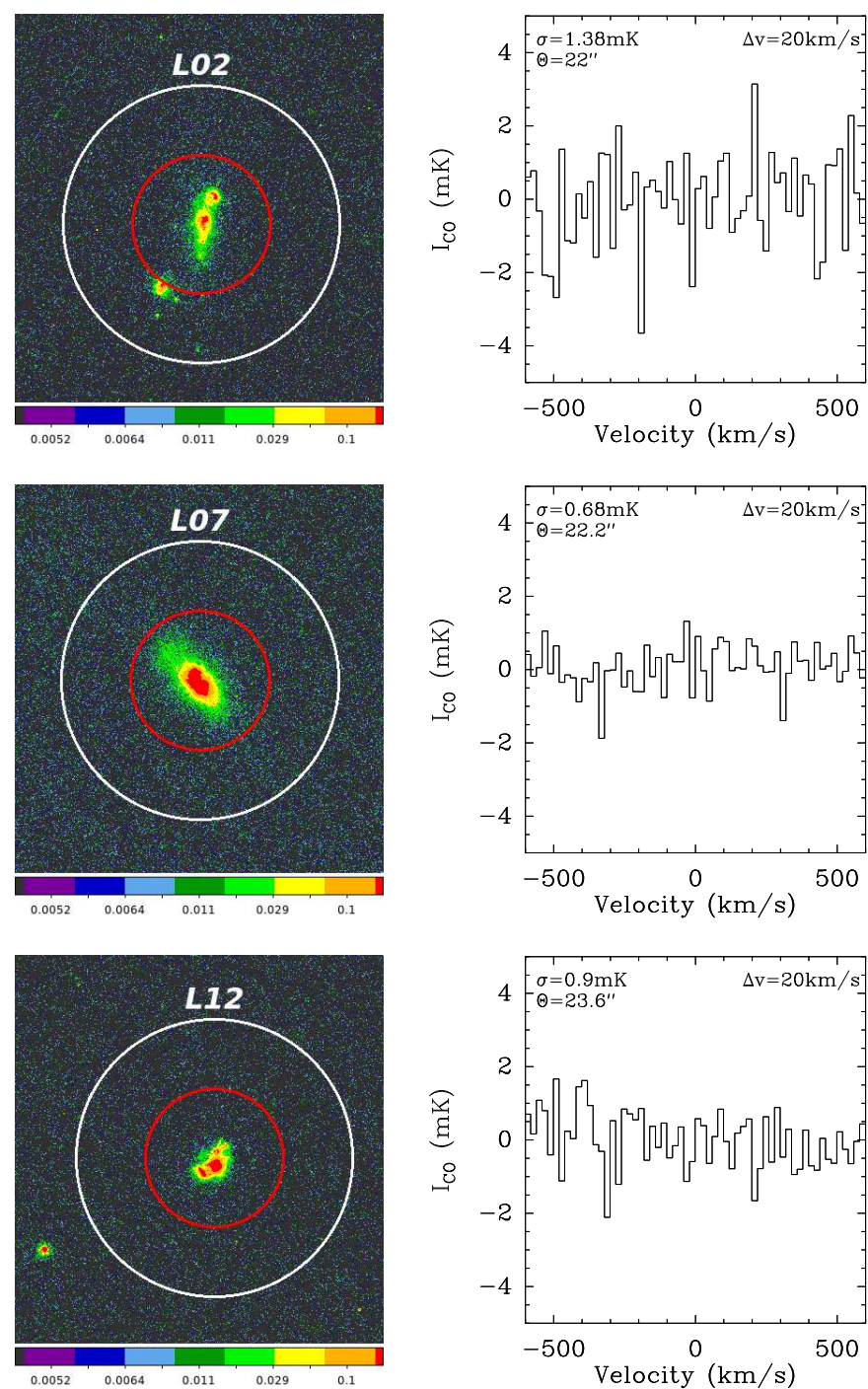

(3) $\mathrm{H} \alpha$ \& HPBW

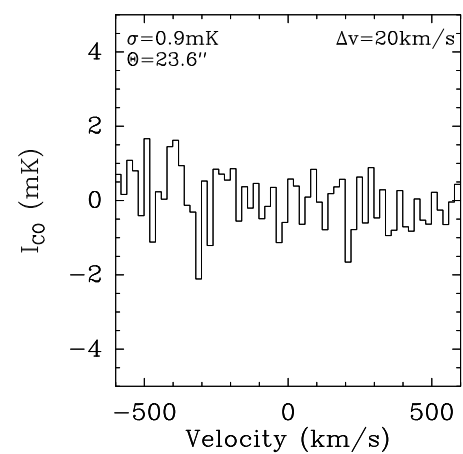

(4) $\mathrm{T}_{\mathrm{MB}}$

Fig. 2. $\mathrm{CO}(1-0)$ non-detections of LARS galaxies. The beam size for the $\mathrm{CO}(1-0)$ transition is shown in Cols. (1) and (3) as white circle on top of our HST H $\alpha$ narrow band images. The measured signals on the main beam brightness temperature scales are shown in Cols. (2) and (4).

All PACS imaging data was reduced using the Herschel Interactive Processing Environment (HIPE; Ott 2010) version 14.0.1. To create a set of homogeneous images of our galaxies, we re-reduced all observations starting from level 1 data products. The mapping was performed using the MADMap maximum likelihood code for LARS 7 and 10, and JScanam otherwise. Due to the different observations available for the galaxies, our final reduction levels range from 2.0 to 3.0, where level 2.0 indicates a fully reduced data set, level 2.5 is the result of combining pairs of observations acquired in the scan and cross-scan mode, and level 3.0 are mosaics of level 2.5 products that belong to the same sky field and observing programme. A summary of the photometric PACS observations used in this paper is shown in Table 4.

In order to measure the fluxes of our galaxies, we used the Daophot algorithm as implemented in the annularSky AperturePhotometry task of HIPE's Jython console. We also examined source sizes and flux measurements using the sourceFitting task. However, since most of our sources are not or only marginally resolved, in particular in the red channel, we finally used the results from the aperture photometry, with an aperture correction factor applied. Our flux measurements are summarized in Table 5.

PACS spectroscopic data of our observing programme were not re-reduced, instead we used the level 2.0 data products provided through the standard PACS spectrometer pipeline. The fluxes of the spectral lines were derived after summation over all spaxels and subsequent fitting of the continuum and the Gaussian line. The continuum subtracted line fluxes were then found from the area below the Gaussian fits. A summary of the measurements is shown in Table 6.

\subsubsection{SOFIA FIR line spectroscopy}

We performed observations of LARS galaxies targeting far infrared (FIR) fine structure lines of [C II] $158 \mu \mathrm{m}$, [O I] $63 \mu \mathrm{m}$, and [O III] $88 \mu \mathrm{m}$ with the Far Infrared Field-Imaging Line Spectrometer (FIFI-LS) aboard the Stratospheric Observatory for Infrared Astronomy (SOFIA) air plane (see Figs. 4-6). Observations were carried out in Cycle 3 under project 03_0059.

Basic data calibration was provided by the FIFI-LS team, that is, level 3 data products, consisting of $\sim 30 \mathrm{~s}$ exposures. The 

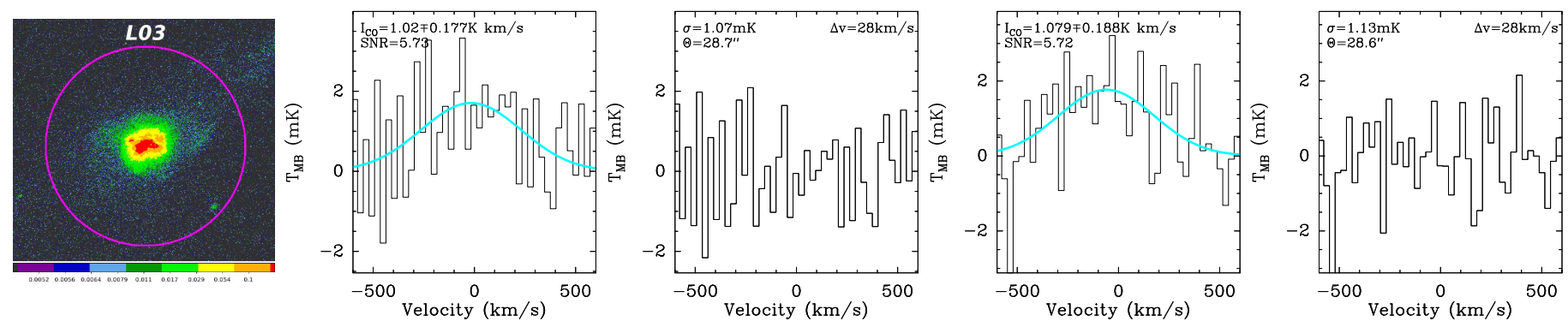

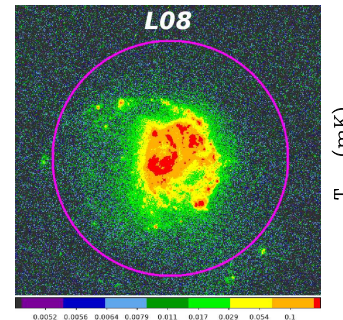

(1) $\mathrm{H} \alpha$ \& HPBW

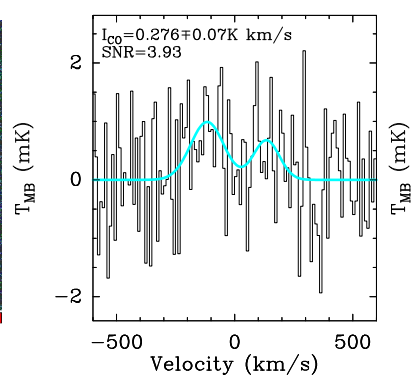

(2) $\mathrm{HCN}(1-0)$

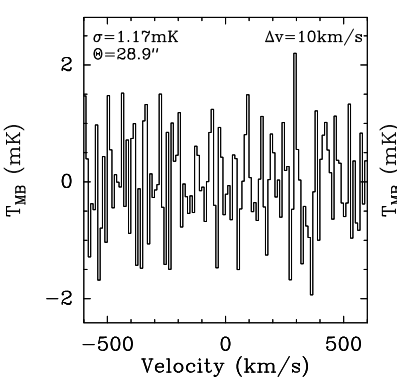

(3) residual

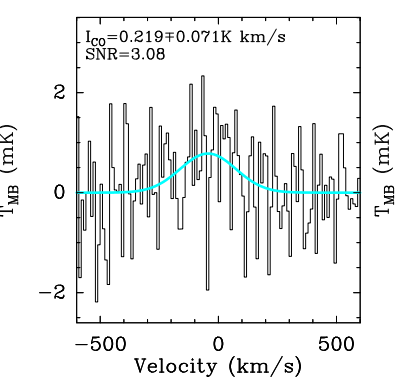

(4) $\mathrm{HCO}^{+}(1-0)$

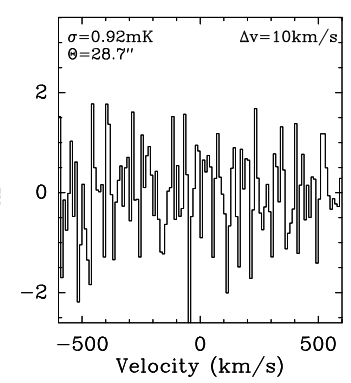

(5) residual

Fig. 3. Detections of dense gas tracers in LARS 3 (top row) and 8 (bottom row). The beam sizes for the transitions are shown in Col. (1) on top of our HST $\mathrm{H} \alpha$ narrow band images. $\mathrm{HCN}$ and $\mathrm{HCO}^{+}(1-0)$ spectra are shown in Cols. (2) and (4). Spectra are given on the $T_{\mathrm{MB}}$ scale in milli Kelvin. Single or double Gaussian fits were performed to calculate the line flux. They are shown as cyan solid lines. The residuals after subtraction of the fits from the data are shown in Cols. (3) and (5).

data were further processed using the same method as described in Smirnova-Pinchukova et al. (2019), which involved an additional selection routine and background subtraction. Final data cubes with a sampling of $6^{\prime \prime}$ were produced with the Drizzle algorithm (Fruchter \& Hook 2002). Total line fluxes were calculated using Gaussian fits to the summed spectra within an aperture of $36^{\prime \prime}$. Spectral ranges with strong atmospheric absorption were masked out for the fitting process. A summary of the measurements is shown in Table 6 . The spectral resolution varies between the channels, with $R \sim 1200\left(\sim 250 \mathrm{~km} \mathrm{~s}^{-1}\right)$ for the observed wavelength of [C II] $158 \mu \mathrm{m}$ and $R \sim 670$ $\left(\sim 450 \mathrm{~km} \mathrm{~s}^{-1}\right)$ for [O III] $88 \mu \mathrm{m}$.

\subsection{3. unWISE source extractions}

The National Aeronautics and Space Administration's Widefield Infrared Survey Explorer (WISE; Wright et al. 2010) mapped the sky at 3.4, 4.6, 12, and $22 \mu \mathrm{m}$ (W1, W2, W3, W4) in 2010, with an angular resolution of 6.1, 6.4, 6.5, and 12.0 arcsec respectively. We checked the WISE all-sky source catalogue for entries of our targets. Unfortunately, photometry was not available for all LARS galaxies. Therefore, we performed source extraction using Sextractor (Bertin \& Arnouts 1996) on unWISE ${ }^{2}$ (Lang 2014) images, which are unblurred coadds of the original WISE imaging. For the extraction, we used the provided weight maps as rms maps, applied Gaussian filters to the W3 and W4 bands in order to smooth the irregular PSFs, and used an rms-based absolute detection threshold. For those LARS galaxies that are unresolved and have a WISE catalogue entry, we find that our extracted flux levels (FLUX_AUTO) agree to a level better than $10 \%$ compared to the catalogue's profile fitting magnitude (mpro). We converted the derived magnitudes to flux densities using W1-W4 zero

\footnotetext{
2 http://unwise.me/
}

Table 4. Summary of PACS data used in this paper.

\begin{tabular}{lllc}
\hline \hline $\begin{array}{l}\text { ID } \\
\text { PACS } \\
\text { obsid }\end{array}$ & $\begin{array}{l}\text { Proposal } \\
\text { code }\end{array}$ & $\begin{array}{c}\text { Reduction } \\
\text { level }\end{array}$ \\
\hline 2 & 1342270859 & OT2_mhayes_4 & 2.0 \\
3 & 1342209025,1342209027 & KPGT_esturm_1 & 3.0 \\
4 & 1342247700 & OT2_mhayes_4 & 2.0 \\
5 & 1342253524,1342253526 & OT2_lhunt_4 & 3.0 \\
6 & 1342261848,1342262223 & OT2_mhayes_4 & 3.0 \\
7 & 1342210558,1342210903, & KPOT_seales01_2 & 3.0 \\
& 1342210946,1342210963, & & \\
& 1342222626 & & \\
8 & 1342248630 & OT2_mhayes_4 & 2.0 \\
9 & 1342270829,1342270830 & OT2_mhayes_4 & 3.0 \\
10 & 1342210567,1342210963, & KPOT_seales01_2 & 3.0 \\
& 1342222626 & & \\
12 & 1342231634,1342231636 & OT1_roverzie_1 & 3.0 \\
13 & 1342237833,1342223573 & OT1_roverzie_1, & 2.5 \\
& & GT1_aconturs_1 & \\
14 & 1342231640,1342231642 & OT1_roverzie_1 & 3.0 \\
\hline
\end{tabular}

magnitude fluxes ${ }^{3}$ of $309.54,171.787,31.674$, and $8.363 \mathrm{Jy}$. The photometric results for the LARS galaxies in units of milli Jansky are summarized in Table 5.

\subsubsection{IRIS photometry}

Only six LARS galaxies were found in the Infrared Astronomical Satellite (IRAS) point source catalogue (PSC) and the faint source catalogue (FSC). Therefore, we downloaded images of

\footnotetext{
3 http://wise2.ipac.caltech.edu/docs/release/allsky/ expsup/sec4_4h.html
} 
Table 5. Wide-field Infrared Survey Explorer and Herschel/PACS photometric measurements of LARS galaxies.

\begin{tabular}{cccccccc}
\hline \hline ID & $\begin{array}{c}\text { W1 } \\
{[\mathrm{mJy}]}\end{array}$ & $\begin{array}{c}\mathrm{W} 2 \\
{[\mathrm{mJy}]}\end{array}$ & $\begin{array}{c}\mathrm{W} 3 \\
{[\mathrm{mJy}]}\end{array}$ & $\begin{array}{c}\mathrm{W} 4 \\
{[\mathrm{mJy}]}\end{array}$ & $\begin{array}{c}\text { P70 } \\
{[\mathrm{mJy}]}\end{array}$ & $\begin{array}{c}\text { P100 } \\
{[\mathrm{mJy}]}\end{array}$ & $\begin{array}{c}\text { P160 } \\
{[\mathrm{mJy}]}\end{array}$ \\
\hline 1 & $1.7 \pm 0.1$ & $1.2 \pm 0.1$ & $14.1 \pm 0.4$ & $97.3 \pm 3.1$ & - & - & - \\
2 & $0.5 \pm 0.1$ & $0.2 \pm 0.1$ & $1.0 \pm 0.3$ & $4.5 \pm 1.5$ & $80 \pm 11$ & - & $121 \pm 14$ \\
3 & $15.5 \pm 0.1$ & $16.7 \pm 0.2$ & $236.3 \pm 3.6$ & $1586.5 \pm 24.0$ & $9306 \pm 8$ & $8628 \pm 7$ & $4745 \pm 19$ \\
4 & $2.0 \pm 0.2$ & $1.4 \pm 0.1$ & $12.5 \pm 0.5$ & $79.9 \pm 2.5$ & $474 \pm 8$ & - & $331 \pm 11$ \\
5 & $0.5 \pm 0.1$ & $0.5 \pm 0.1$ & $4.5 \pm 0.7$ & $29.1 \pm 5.5$ & $113 \pm 5$ & $117 \pm 4$ & $40 \pm 4$ \\
6 & $0.4 \pm 0.2$ & $0.4 \pm 0.2$ & - & - & $51 \pm 8$ & $24 \pm 8$ & $58 \pm 10$ \\
7 & $0.9 \pm 0.1$ & $0.8 \pm 0.1$ & $8.1 \pm 0.5$ & $51.9 \pm 2.1$ & - & $303 \pm 78$ & $309 \pm 50$ \\
8 & $16.1 \pm 0.1$ & $11.3 \pm 0.1$ & $108.7 \pm 1.2$ & $323.5 \pm 3.3$ & - & $4322 \pm 27$ & $3346 \pm 34$ \\
9 & $2.3 \pm 0.5$ & $4.0 \pm 0.1$ & $36.2 \pm 0.6$ & $171.6 \pm 4.1$ & $1147 \pm 17$ & $1415 \pm 20$ & $1306 \pm 27$ \\
10 & $1.0 \pm 0.1$ & $0.8 \pm 0.2$ & $3.4 \pm 0.6$ & $9.3 \pm 3.2$ & - & $336 \pm 77$ & $413 \pm 50$ \\
11 & $4.1 \pm 0.3$ & $3.1 \pm 0.1$ & $27.9 \pm 0.7$ & $56.7 \pm 4.9$ & - & - & - \\
12 & $0.3 \pm 0.2$ & $0.3 \pm 0.1$ & $2.8 \pm 0.6$ & $17.4 \pm 3.5$ & $104 \pm 4$ & $113 \pm 6$ & $62 \pm 4$ \\
13 & $0.6 \pm 0.1$ & $1.1 \pm 0.2$ & $10.6 \pm 0.7$ & $39.7 \pm 4.5$ & $506 \pm 3$ & $464 \pm 3$ & $286 \pm 3$ \\
14 & $0.2 \pm 0.1$ & $0.1 \pm 0.1$ & $0.3 \pm 0.2$ & $2.8 \pm 0.7$ & $16 \pm 2$ & $12 \pm 1$ & - \\
\hline
\end{tabular}

Table 6. Summary of Gaussian fit results for the PACS and FIFI-LS spectral lines of [C II]158, [O I]63, and [O III]88.

\begin{tabular}{llllll}
\hline \hline ID & Line & $\begin{array}{l}\text { Centre } \\
{\left[\mathrm{km} \mathrm{s}^{-1}\right]}\end{array}$ & $\begin{array}{l}F W H M \\
{\left[\mathrm{~km} \mathrm{~s}^{-1}\right]}\end{array}$ & $\begin{array}{l}\text { Flux } \\
{\left[10^{-15} \mathrm{erg} \mathrm{s}^{-1} \mathrm{~cm}^{-2}\right]}\end{array}$ & Instrument \\
\hline 2 & {$[$ C II $] 158$} & -20 & 209 & $8.4 \pm 4.0$ & PACS \\
2 & {$[\mathrm{O} \mathrm{I}] 63$} & 177 & $<572$ & $<30.3$ & PACS \\
3 & {$[$ C II $] 158$} & -52 & 398 & $688.0 \pm 15.6$ & PACS \\
3 & {$[$ O I $] 63$} & 3 & 380 & $1317.0 \pm 63.8$ & PACS \\
3 & {$[$ I III $] 88$} & -43 & 440 & $843.8 \pm 54.7$ & PACS \\
5 & {$[$ C II $] 158$} & -117 & 584 & $479.1 \pm 84.7$ & FIFI-LS \\
5 & {$[$ O III $] 88$} & -110 & $>222(\dagger)$ & $254.9 \pm 43.3$ & FIFI-LS \\
8 & {$[$ C II $] 158$} & -20 & 497 & $1052.0 \pm 161.3$ & FIFI-LS \\
9 & {$[$ C II $] 158$} & 105 & 284 & $285.7 \pm 5.7$ & PACS \\
9 & {$[$ O I $] 63$} & 153 & 218 & $249.3 \pm 23.1$ & PACS \\
11 & {$[$ C II $] 158$} & -118 & 388 & $502.3 \pm 30.5$ & FIFI-LS \\
12 & {$[$ C II $] 158$} & 38 & 214 & $14.5 \pm 2.1$ & PACS \\
12 & {$[$ O I $] 63$} & 134 & 212 & $20.1 \pm 6.0$ & PACS \\
13 & {$[$ C II $] 158$} & -18 & 299 & $30.8 \pm 3.1$ & PACS \\
13 & {$[$ O I $] 63$} & 127 & 280 & $36.6 \pm 7.7$ & PACS \\
\hline
\end{tabular}

Notes. ${ }^{(\dagger)}$ Due to strong atmospheric absorption, the red side of the line is truncated and the reported value is considered to be a lower limit. Also we note that the spectral resolution varies between the different instruments and channels. PACS offers spectral resolutions of $\sim 240 \mathrm{~km} \mathrm{~s}^{-1}$ for the [C II] $158 \mu \mathrm{m}$ observations and $\sim 100 \mathrm{~km} \mathrm{~s}^{-1}$ for [O I] $63 \mu \mathrm{m}$. FIFI-LS has $R \sim 1200\left(\sim 250 \mathrm{~km} \mathrm{~s}^{-1}\right)$ for the observed wavelength of [C II] $158 \mu \mathrm{m}$ and $R \sim 670\left(\sim 450 \mathrm{~km} \mathrm{~s}^{-1}\right)$ for $[\mathrm{O} \mathrm{III}] 88 \mu \mathrm{m}$.

the improved reprocessing of the IRAS Survey (IRIS ${ }^{4}$ ), and performed photometric flux measurements for all LARS galaxies using these data products. The originally downloaded IRIS images cover fields of two square degrees (NASA's Montage tool $^{5}$ was used to create smaller cutouts). The full width at half maximum of the PSF in the new images is 3.8, 3.8, 4.0, and 4.3 arcmin for the four IRAS bands of $12,25,60$, and $100 \mu \mathrm{m}$. All LARS galaxies are thus unresolved. After conversion from mega Jansky per steradian to milli Jansky per pixel, flux densities were extracted using SExtractor's (Bertin \& Arnouts 1996) FLUX_AUTO value. The results are shown in Table 7.

\footnotetext{
4 http://irsa.ipac.caltech.edu/data/IRIS/index cutouts.html

5 http://montage.ipac.caltech.edu/
}

\subsubsection{AKARI photometry}

We also checked the archive of the AKARI (Murakami et al. 2007) infrared satellite mission for LARS galaxy data. AKARI comprises two main instruments with 13 spectral bands in total covering a wavelength range of $1.8-180 \mu \mathrm{m}$. For our purpose we focused on the four FIS (Far-Infrared Surveyor, Kawada et al. 2007) bands with central wavelengths of $65,90,140$, and $160 \mu \mathrm{m}$. The fluxes of only three LARS galaxies were published in the FIS Bright Source Catalogue Version 2.0 (released April 2016), so we downloaded cutouts of the AKARI far-infrared allsky survey maps (Doi et al. 2015; Takita et al. 2015). The originally downloaded images cover fields of six degrees (NASA's Montage tool ${ }^{5}$ was used to create smaller cutouts). The full width at half maximum of the PSF in these maps is roughly 60 arcsec for the 65 and the $90 \mu \mathrm{m}$ bands and 90 arcsec for the 140 and $160 \mu \mathrm{m}$ bands. All LARS galaxies are thus unresolved. 

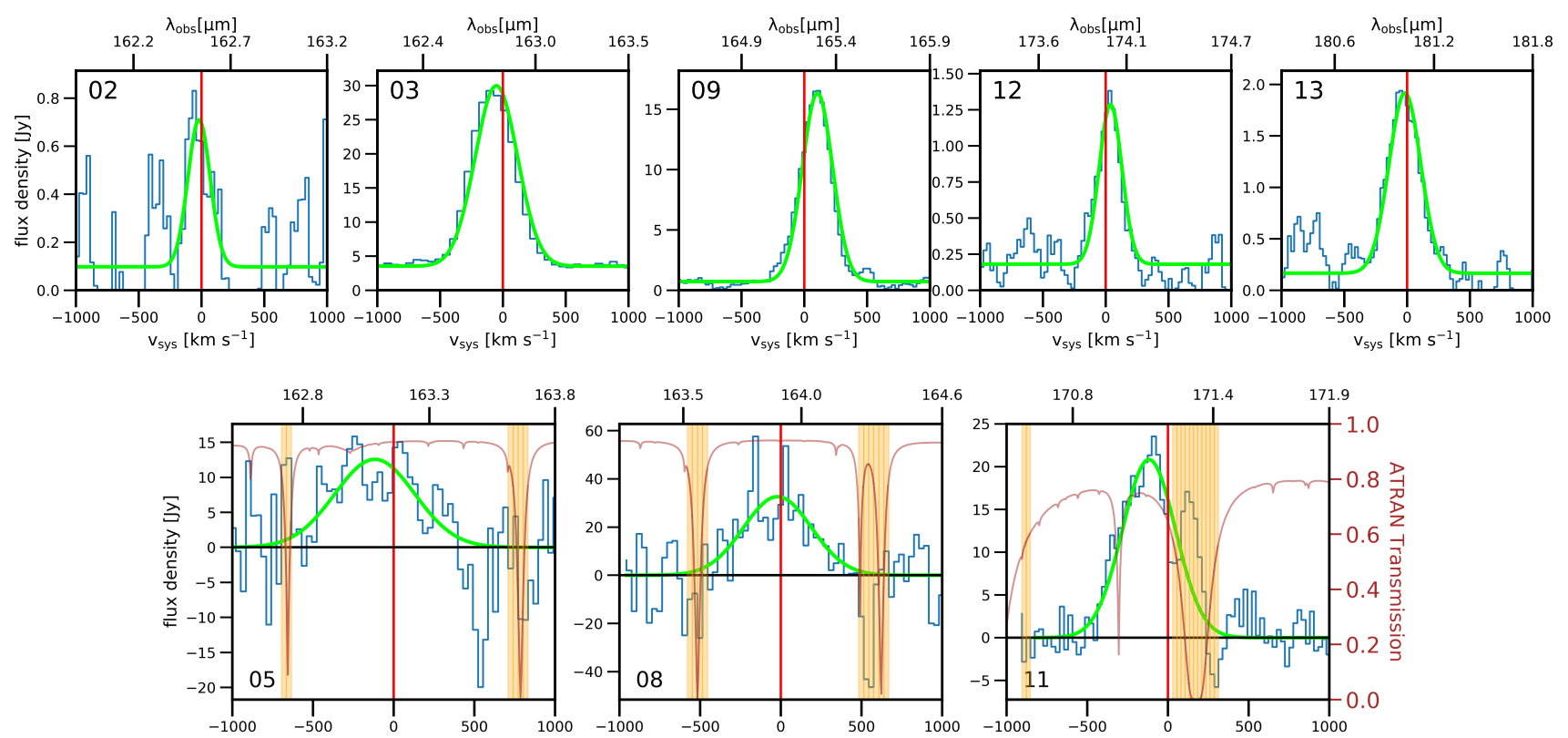

Fig. 4. [C II] $158 \mu \mathrm{m}$ fine structure lines of LARS galaxies. Herschel/PACS spectra are shown in the top row, and SOFIA/FIFI-LS spectra in the bottom row. Total fluxes were derived using Gaussian fits (see Table 6). For FIFI-LS data, the atmospheric absorption, calculated using ATRAN (Lord 1992), is shown as a red curve. Spectral ranges with strong atmospheric absorption (see orange range) were masked out for the fitting process. The zero-velocity (red vertical line) is related to the H I systemic velocity from Pardy et al. (2014) for all LARS galaxies but LARS 12-14 (which have no H I detection). For those, the systemic velocity is based on SDSS optical redshifts.

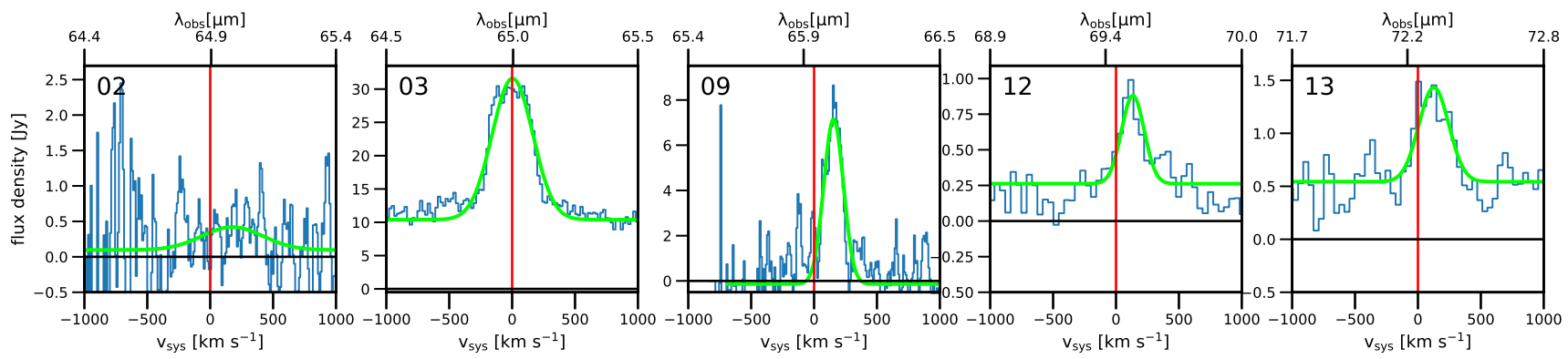

Fig. 5. [O I] $63 \mu \mathrm{m}$ fine structure lines of LARS galaxies, obtained with Herschel/PACS. Total fluxes were derived using Gaussian fits (see Table 6). The zero-velocity (red vertical line) is related to the H I systemic velocity from Pardy et al. (2014) for all LARS galaxies except LARS 12-14 (which have no H I detection). For those, the systemic velocity is based on SDSS optical redshifts.

After conversion from mega Jansky per steradian to milli Jansky per pixel, flux densities were extracted using SExtractor's (Bertin \& Arnouts 1996) FLUX_AUTO value. The results are summarized in Table 7.

\subsection{Previously derived Lyman alpha related quantities: Lyman alpha escape fraction and scattering length}

Hayes et al. (2014) computed the Ly $\alpha$ escape fractions of LARS galaxies in the following way. Assuming case B recombination theory, the observed Ly $\alpha$ flux was compared to that expected from extinction-corrected $\mathrm{H} \alpha$. We note that $\mathrm{H} \alpha$ was obtained through HST narrow band imaging, meaning all of the ionised gas is captured. Adopting an intrinsic value of $\operatorname{Ly} \alpha_{\text {intrinsic }} / \mathrm{H} \alpha=8.7$, the escape fraction was calculated as Ly $\alpha_{\text {obs }} / \mathrm{Ly} \alpha_{\text {intrinsic }}$.

The Ly $\alpha$ scattering lengths of LARS galaxies were published by Bridge et al. (2018). They are derived from modelling the Ly $\alpha$ morphology based on $\mathrm{H} \alpha$ images, which again when multiplied by 8.7 should give the approximate intrinsic morphology of Ly $\alpha$. The scattering is then modelled using a Gaussian convolution, with an additional component of dust attenuation (from $\mathrm{H} \alpha / \mathrm{H} \beta$ ). Using the Markov chain Monte Carlo (MCMC) method, the observed Ly $\alpha$ image is reproduced and the scattering length is found from the size of the best-fit Gaussian convolution kernel.

\section{Methods}

In this section we outline the dust models from which physical quantities (dust mass, dust temperature, radiation field strength, star formation rates) are derived. Prescriptions used to convert $\mathrm{CO}$ observations and dust masses to molecular gas masses are explained.

\subsection{Dust-based gas masses}

Given that we could detect only one third of the LARS sample in $\mathrm{CO}(1-0)$, we employed the models ${ }^{6}$ of Draine \& Li (2007) to derive dust masses, which finally enables us to infer the

6 ftp://ftp.astro.princeton.edu/draine/dust/irem4/ 

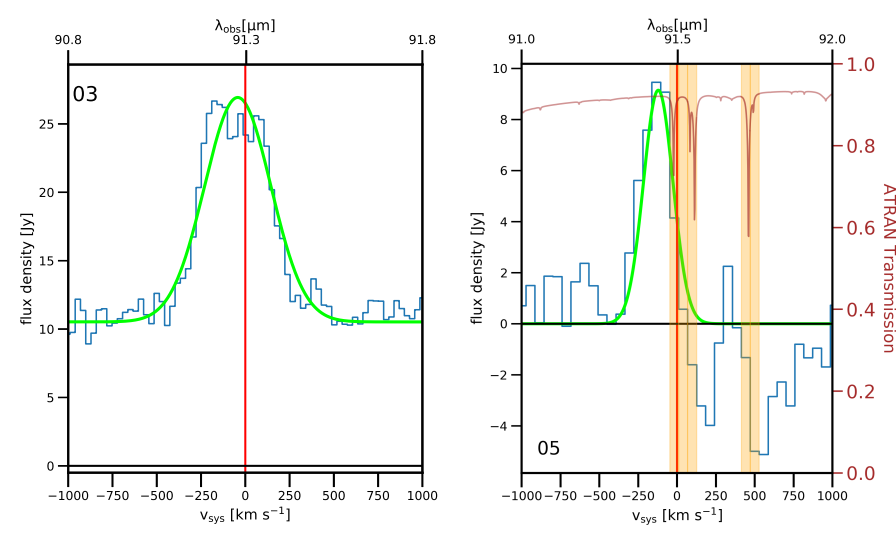

Fig. 6. [O III] $] 88 \mu \mathrm{m}$ fine structure lines of LARS 3 (left) and 5 (right), obtained with Herschel/PACS and SOFIA/FIFI-LS respectively. Total fluxes were derived using Gaussian fits (see Table 6). For FIFI-LS data, the atmospheric absorption, calculated using ATRAN (Lord 1992), is shown as a red curve. Spectral ranges with strong atmospheric absorption (see orange range) were masked out for the fitting process. The zero-velocity (red vertical line) is related to the H I systemic velocity from Pardy et al. (2014).

total gas masses for all LARS galaxies in a homogeneous way. The models predict infrared spectra for wavelengths between $1 \mu \mathrm{m}$ and $1 \mathrm{~cm}$. They are based on a mixture of dust grains containing carbonaceous grains, including Polycyclic Aromatic Hydrocarbons (PAHs) and amorphous silicates, with grain size distributions - or extinction curves - as found in the Milky Way (MW), the Large Magellanic Cloud (LMC), and the Small Magellanic Cloud (SMC). Within each distribution type (MW, LMC, or SMC), a variety of PAH dust mass fractions $q_{\mathrm{PAH}}$ is implemented, ranging from $0.1 \%$ to $4.6 \%$, with the lowest and highest $q_{\mathrm{PAH}}$ value being consistent with observations of the dust in the SMC and MW, respectively. The total power radiated per $\mathrm{H}-$ nucleon per unit frequency per steradian, $j_{v}$, then depends on two radiative components: (1) the average stellar intensity found in the diffuse ISM, described by the energy density parameter $U_{\min }$, and (2) a fraction $\gamma$ of dust mass that is exposed to a power law distribution of intensities ranging from $U_{\min }$ to $U_{\max }$ with a power law slope $\alpha=-2$. The latter component is related to PDRs. The radiation field parameter $U$ is a scale factor to the local Galactic interstellar radiation field estimated by Mathis et al. (1983), and can be related to the Habing (1968) field $G_{0}$ via $U=0.88 G_{0}$. The final value of $j_{v}$ is given by a linear combination of the two components with a $(1-\gamma)$ mass fraction that originates from dust exposed to the average, diffuse stellar intensity and a fraction $\gamma$ related to the currently ongoing star formation, with $0 \leq \gamma \leq 1$. Thus, the fraction of dust mass associated with PDR regions is given by the product of $\gamma$ and $M_{\text {dust }}$.

Another parameter included in the models is the solid angle subtended by (old) stars, $\Omega_{*}$, since they also contribute to the overall emission spectrum, albeit mainly at wavelengths of a few $\mu \mathrm{m}$, which is at the low end of the range we are considering. Assuming an average stellar surface temperature $T$ of $5000 \mathrm{~K}$, their share is calculated through the Planck function $B_{v}(T)$.

In the analysis that follows we keep the upper energy density cutoff $U_{\max }$ fixed at a value of $10^{6}$, which decreases the number of degrees of freedom by 1 , while only having a small influence on the fitting result, as shown by Draine et al. (2007). Hence, we are left with five free parameters for the models: $q_{\mathrm{PAH}}, U_{\mathrm{min}}, \gamma$, $M_{\text {dust }}$, and $\Omega_{*}$.
We derive those quantities for LARS galaxies using a Bayesian inference, similar to the method described in Galliano (2018). Our logarithmic prior is set to a constant (0.0) within the following intervals for our parameters: $U_{\min }: 0.1-25$, $q_{\mathrm{PAH}}: 0.1-4.6 \%, M_{\text {dust }}: 10^{6}-10^{9} M_{\odot}, \gamma: 10^{-3}-10^{-0.1}$, and $\Omega_{*}$ : $10^{-10}-10^{-7}$ steradian. Outside these intervals, the prior is set to negative infinity. Using the photometric data from WISE, Herschel/PACS, AKARI, and IRAS allows us to infer the logarithmic likelihood for the whole parameter space. We note that - besides the measured photometric error - a $10 \%$ model-based error is also included when calculating the logarithmic likelihood of the parameter space. The latter is explored through the MCMC method as implemented in the Python package emcee (version 3.0.2). The final joint posterior probability distribution of the derived parameters is shown in Appendix A. The bestfit spectral energy distribution (SED) was found from the $50 \%$ quantile of the logarithmic posterior distribution and the uncertainties are derived from a quantile-based credible interval corresponding to $16 \%$ and $84 \%$ (i.e. $\pm 1 \sigma$ ). A summary of the derived quantities is found in Table 8 , where the number of degrees of freedom $(v)$ is also denoted. The dust masses are finally converted into total gas mass estimates $\left(M_{\mathrm{gas}_{\mathrm{est}}}\right)$ using a metallicitydependent gas-to-dust ratio as described in the following section.

\subsubsection{Metallicity-dependent gas-to-dust ratio}

Once the dust masses are calculated, we convert them into total gas masses using a metallicity-dependent gas-to-dust ratio (GDR). The GDR is found to scale with $Z^{-1}$ at near-solar metallicities (Lisenfeld \& Ferrara 1998; Hirashita et al. 2002; James et al. 2002; Hunt et al. 2005; Draine et al. 2007; Engelbracht et al. 2008; Galliano et al. 2008; Muñoz-Mateos et al. 2009; Bendo et al. 2010; Galametz et al. 2011; Magrini et al. 2011; Sandstrom et al. 2013; Rémy-Ruyer et al. 2014; Roman-Duval et al. 2014; Cortese et al. 2016; Kahre et al. 2018). However, this trend is not seen in low-metallicity dwarf galaxies, which show higher GDRs than expected (Lisenfeld \& Ferrara 1998; Galliano et al. 2003, 2005, 2011; Galametz et al. 2011; RémyRuyer et al. 2014; Kahre et al. 2018). Given the generally low metal content of LARS galaxies, ranging from $\sim 13 \%$ solar to $\sim 2 / 3$ solar, we adopt a metallicity-dependent GDR as defined in Rémy-Ruyer et al. (2014), which is a broken power law, that is, a linear scaling down to a metallicity of $12+\log (\mathrm{O} / \mathrm{H})=8.1$, and a power law with an index of 3.1 at even lower $Z$. For $Z=12+\log (\mathrm{O} / \mathrm{H})>8.10$,

$\log (\mathrm{GDR})=2.21+1.0 \times(8.69-Z)$,

and for $Z=12+\log (\mathrm{O} / \mathrm{H}) \leq 8.10$,

$\log (\mathrm{GDR})=0.96+3.1 \times(8.69-Z)$.

Several other prescriptions for GDR calculations have been published to date. We discuss the reasoning for our choice in Appendix E.

\subsubsection{Far-infrared SEDs of LARS galaxies}

Spectral energy distribution fits for all LARS galaxies are shown in Appendix B. The agreement between the models and the data is generally very good, in particular below $\sim 100 \mu \mathrm{m}$. However, due to the lack of observations at wavelengths longer than $160 \mu \mathrm{m}$, the uncertainty largely increases to this end. The fit for LARS 6 is somewhat unreliable, owing to the fact that it 
Table 7. IRAS (I) and AKARI (A) photometric measurements of LARS galaxies.

\begin{tabular}{ccccccccc}
\hline \hline ID & $\begin{array}{c}\text { I12 } \\
{[\mathrm{mJy}]}\end{array}$ & $\begin{array}{c}\text { I25 } \\
{[\mathrm{mJy}]}\end{array}$ & $\begin{array}{c}\text { I60 } \\
{[\mathrm{mJy}]}\end{array}$ & $\begin{array}{c}\text { I100 } \\
{[\mathrm{mJy}]}\end{array}$ & $\begin{array}{c}\text { A65 } \\
{[\mathrm{mJy}]}\end{array}$ & $\begin{array}{c}\text { A90 } \\
{[\mathrm{mJy}]}\end{array}$ & $\begin{array}{c}\text { A140 } \\
{[\mathrm{mJy}]}\end{array}$ & $\begin{array}{c}\text { A165 } \\
{[\mathrm{mJy}]}\end{array}$ \\
\hline 1 & - & - & $810 \pm 257$ & - & - & $384 \pm 146$ & - & - \\
3 & $307 \pm 108$ & $1694 \pm 203$ & $11461 \pm 733$ & $15350 \pm 4109$ & $7663 \pm 1054$ & $9249 \pm 485$ & $3311 \pm 200$ & $1552 \pm 251$ \\
4 & - & - & - & - & - & $321 \pm 197$ & $218 \pm 148$ & - \\
6 & - & - & - & - & - & $222 \pm 163$ & - \\
8 & - & - & $4096 \pm 464$ & $10633 \pm 4930$ & $1488 \pm 617$ & $3593 \pm 689$ & $3769 \pm 1786$ & $3441 \pm 2531$ \\
9 & - & - & $1085 \pm 209$ & $1979 \pm 907$ & - & $1022 \pm 246$ & - & - \\
11 & - & - & $505 \pm 209$ & $1929 \pm 1057$ & - & $810 \pm 272$ & $859 \pm 155$ & - \\
13 & - & - & - & - & - & $298 \pm 194$ & - & - \\
\hline
\end{tabular}

Table 8. Results from Bayesian inference of Draine \& Li (2007) dust model parameters for LARS galaxies.

\begin{tabular}{|c|c|c|c|c|c|c|c|}
\hline ID & $q_{\mathrm{PAH}}$ & $\begin{array}{c}\log \left(\Omega_{*}\right) \\
{[\mathrm{sr}]}\end{array}$ & $\begin{array}{c}U_{\min } \\
\left(U=0.88 G_{0}\right)\end{array}$ & $\log (\gamma)$ & $\begin{array}{c}\log \left(M_{\text {dust }}\right) \\
{\left[M_{\odot}\right]}\end{array}$ & $\begin{array}{c}T_{\text {dust }} \\
{[\mathrm{K}]} \\
\end{array}$ & $v$ \\
\hline 1 & $0.86_{-0.21}^{+0.74}$ & $-8.96_{-0.21}^{+0.21}$ & $7.37_{-4.64}^{+8.07}$ & $-0.68_{-0.27}^{+0.27}$ & $7.40_{-0.66}^{+0.58}$ & $25.11_{-3.83}^{+3.29}$ & 1 \\
\hline 2 & $0.83_{-0.32}^{+0.64}$ & $-9.54_{-0.28}^{+0.13}$ & $\begin{array}{r}8.45_{-3.49}^{+6.84} \\
\end{array}$ & $-2.28_{-0.80}^{+0.38}$ & $\begin{array}{r}6.86_{-0.56}^{+0.00} \\
\text {.0. }\end{array}$ & $25.69_{-2.18}^{+2.68}$ & 1 \\
\hline 3 & $1.21_{-0.67}^{+0.92}$ & $\begin{array}{r}-0.09_{-0.44}^{+0.16} \\
-0.28\end{array}$ & $12.73_{-2.97}^{+5.29}$ & $\begin{array}{r}-0.80 \\
-0.80_{-0.14}^{+0.19}\end{array}$ & $\begin{array}{r}8.01_{-0.20}^{+0.50} \\
0.26\end{array}$ & $29.15_{-1.19}^{+1.64}$ & 10 \\
\hline 4 & $1.17_{-0.44}^{-0.0 /}$ & $\begin{array}{r}-0.44 \\
-8.91_{-0.16}^{+0.15}\end{array}$ & $6.67_{-1.83}^{+2.04}$ & $\begin{array}{r}-0.14 \\
-0.87_{-0.17}^{+0.19}\end{array}$ & $7.13_{-0.18}^{+0.51}$ & $\begin{array}{r}24.70_{-1.29}^{+1.19}\end{array}$ & 3 \\
\hline 5 & $1.71_{-0.61}^{+0.44}$ & $\begin{array}{l}-0.16 \\
-9.42_{-0.18}^{+0.21}\end{array}$ & $18.14_{-8.93}^{-1.83}$ & $\begin{array}{r}-1.11_{-0.33}^{+0.37} \\
-{ }^{+0.35}\end{array}$ & $\begin{array}{r}6.01_{-0.66}^{+0.938} \\
\end{array}$ & $29.18_{-3.12}^{+1.67}$ & 2 \\
\hline 6 & $3.27_{-1.71}^{+1.46}$ & $\begin{array}{r}-9.67_{-0.80}^{+0.10} \\
-0.35 \\
\end{array}$ & $10.74_{-4.95}^{+3.57}$ & $-1.91_{-1.23}^{+1.11}$ & $5.73_{-0.64}^{+0.61}$ & $26.74_{-2.62}^{+1.31}$ & 1 \\
\hline 7 & $1.23_{-0.59}^{+1.11}$ & $\begin{array}{r}-9.21_{-0.15}^{+0.16} \\
-9.00\end{array}$ & $9.34_{-5.04}^{+5.90}$ & $\begin{array}{r}-1.23 \\
-0.84_{-0.22}^{+0.17}\end{array}$ & $\begin{array}{r}-0.04 \\
7.20_{-0.45}^{+0.50}\end{array}$ & $26.12_{-3.17}^{+2.19}$ & 1 \\
\hline 8 & $1.64_{-108}^{+0.99}$ & $\begin{array}{r}-0.133_{-014}^{+0.18} \\
-\end{array}$ & $9.09_{-3.13}^{+3.04}$ & $-1.34_{-0.11}^{+0.22}$ & $8.14_{-0.17}^{-0.45}$ & $24.24_{-1.75}^{+1.57}$ & 7 \\
\hline 9 & $1.78_{-0.67}^{+-0.68}$ & $\begin{array}{r}-0.14 \\
-8.54_{-0.08}^{+0.10}\end{array}$ & $\begin{array}{r}6.58_{-2.76}^{+3.43} \\
0\end{array}$ & $\begin{array}{r}-07_{-0.16}^{-0.21} \\
-1.02\end{array}$ & $7.95_{-0.18}^{+0.56}$ & $24.64_{-2.13}^{+1.35}$ & 5 \\
\hline 10 & $1.36_{-0.38}^{+0.6 /}$ & $\begin{array}{r}-0.08 \\
-8.99_{-0.10}^{+0.09}\end{array}$ & $\begin{array}{r}-2.16 \\
7.15_{-4.25}^{+6.36}\end{array}$ & $\begin{array}{r}-0.16 \\
-2.35_{-0.81}^{+0.50}\end{array}$ & $\begin{array}{r}-0.18 \\
7.63_{-0.28}^{+0.38}\end{array}$ & $24.98_{-3.49}^{+2.80}$ & 1 \\
\hline 11 & $4.38_{-1.66}^{+0.38}$ & $-8.48_{-0.10}^{+0.09}$ & $\begin{array}{r}-4.25 \\
7.30_{-5.00}^{+6.81}\end{array}$ & $\begin{array}{r}-0.81 \\
-1.43_{-0.29}^{+0.29}\end{array}$ & $8.25_{-0.033}^{-0.28}$ & $25.07_{-4.39}^{+2.91}$ & 3 \\
\hline 12 & $\begin{array}{r}2.70_{-1.44}^{+1.66} \\
\end{array}$ & $-10.20_{-1.01}^{-0.10}$ & $\begin{array}{r}17.70_{-5.77}^{+5.00} \\
1.48\end{array}$ & $\begin{array}{r}-0.29 \\
-0.94_{-0.24}^{+0.30}\end{array}$ & $\begin{array}{r}-0.03 \\
7.08_{-0.12}^{+0.17}\end{array}$ & $\begin{array}{r}-4.39 \\
29.06_{-1.85}^{+1.55}\end{array}$ & 2 \\
\hline 13 & $\begin{array}{r}-1.44 \\
3.47_{-0.92}^{+1.00}\end{array}$ & $-9.43_{-0.32}^{+0.01}$ & $19.22_{-4.97}^{+7.02}$ & $\begin{array}{r}-0.24 \\
-1.11_{-0.24}^{+0.11}\end{array}$ & $8.11_{-0.15}^{+0.15}$ & $29.46_{-1.43}^{+1.85}$ & 3 \\
\hline 14 & $2.81_{-1.47}^{+0.52}$ & $\begin{array}{r}-0.32 \\
-10.41_{-0.97}^{+0.52}\end{array}$ & $11.34_{-7.25}^{-8.98}$ & $\begin{array}{r}-0.68_{-0.75}^{-0.24} \\
-0.64\end{array}$ & $\begin{array}{r}6.90_{-0.28}^{+0.15} \\
6.04\end{array}$ & $26.98_{-4.22}^{-1.43}$ & 1 \\
\hline
\end{tabular}

is the faintest galaxy in the PACS bands of the whole sample. An examination of the shape of the SEDs shows that while most galaxies have a rather flat distribution in the range between 50-100 $\mu \mathrm{m}$, LARS 2 and 10 show significant steepening within that range. This is a result of the lack of hot dust directly associated with star formation that would show up as excess in the midinfrared. Consequently, they have lower $\gamma$ values, which denotes the fraction of dust mass linked to PDR regions.

We further caution that our flux measurements were obtained using varying aperture sizes, reflecting the capabilities of the different instruments at varying wavelengths. In particular, a single source in IRAS or AKARI may show up as several sources in the blue WISE bands. In most cases the latter are likely of stellar origin and thus do not have an impact on measurements in the far infrared. However, some might still be of extra galactic origin, contaminating our measurements and SED fits.

\section{2. $\mathrm{CO}(1-0)$-based molecular masses}

In the following, a description of how observed $\mathrm{CO}$ intensities were transformed into molecular gas masses is given. In particular, we describe the aperture correction and the metallicitydependent conversion factor.

\subsection{1. $\mathrm{CO}(1-0)$ luminosity}

We convert our measured $\mathrm{CO}(1-0)$ velocity-integrated main beam brightness temperatures $I_{\mathrm{CO}}$ (given in units of Kelvin kilometer per second) to $\mathrm{CO}$ luminosities using the definition of $L_{\mathrm{CO}}^{\prime}$ by Solomon \& Vanden Bout (2005), with an aperture correction factor added:

$L_{\mathrm{CO}}^{\prime}=C_{\mathrm{AP}} * 23.5 \Omega I_{\mathrm{CO}}(1+z)^{-3} D_{\mathrm{L}}^{2}$.

The line luminosity $L_{\mathrm{CO}}^{\prime}$ is given in $\mathrm{K} \mathrm{km} \mathrm{s}^{-1} \mathrm{pc}^{2}, \Omega$ is the solid angle of the Gaussian beam in $\operatorname{arcsec}^{2}, z$ the redshift, and $D_{\mathrm{L}}$ the luminosity distance in mega parsec. In the original formula found in Solomon \& Vanden Bout (2005) (Eq. (2) therein), $\Omega$ is the solid angle of the source convolved with the beam. In our case, however, we perform this convolution within the calculation of the aperture correction factor and thus must use the solid angle of the Gaussian beam instead.

\subsubsection{Aperture correction for the $\mathrm{CO}(1-0)$ data}

Although the majority of the galaxies in our sample is smaller than the beam size $\theta$ of the IRAM 30m telescope, some galaxies have blue diameters, $D_{25}$, much larger than $\theta$ (compare Tables 1 and 2 and Figs. 1 and 2). In order to estimate the total molecular 
masses of those, an aperture correction is needed. We employ a similar method as described in Lisenfeld et al. (2011) and Stark et al. (2013), which we briefly outline here. Assuming an exponential $\mathrm{CO}$ source brightness distribution, the total $\mathrm{CO}$ flux of a circular $(\theta=0 \ldots 2 \pi)$ galaxy can be written in polar coordinates as

$I_{\mathrm{CO}, \mathrm{extra}}=\int_{0}^{2 \pi} \int_{0}^{\infty} I(r) 2 \pi r \mathrm{~d} r \mathrm{~d} \theta$.

Assuming $I_{\mathrm{CO}}(r)$ is exponentially distributed with a central peak $I_{0}$ and a scale length of $r_{\mathrm{e}, \mathrm{CO}}$, the above equation can be rewritten as

$I_{\mathrm{CO}, \text { extra }}=I_{0} \int_{0}^{2 \pi} \int_{0}^{\infty} \exp \left[-\left(\frac{r}{r_{\mathrm{e}, \mathrm{CO}}}\right)\right] 2 \pi r \mathrm{~d} r \mathrm{~d} \theta$.

This integral can be solved by partial integration and $I_{\mathrm{CO} \text {,extra }}$ is found to be

$I_{\mathrm{CO}, \text { extra }}=I_{0} 2 \pi r_{\mathrm{e}, \mathrm{CO}}^{2}$.

The shape of the main beam can be typically estimated as a Gaussian. In fact, it represents a weighting function, which attenuates the source flux. Its 2D Cartesian formula for a unity peak height is

$G(x, y)=\exp \left[-\left(\frac{x^{2}}{\sigma_{x}^{2}}+\frac{y^{2}}{\sigma_{y}^{2}}\right)\right]$.

The standard deviation $\sigma$ is related to the full width at half maximum or rather the half power beam width $(\theta)$ by

$\theta=2 \sqrt{2 \ln (2)} \sigma$.

Since the measured flux is the result of a convolution of the source brightness distribution with a Gaussian, the observed flux within the beam can be expressed as

$$
\begin{aligned}
I_{\mathrm{CO}, \mathrm{obs}}= & I_{\mathrm{CO}, \operatorname{extra}} * G(x, y) \\
= & 4 I_{0} \int_{0}^{\infty} \int_{0}^{\infty} \exp \left[-\frac{\sqrt{x^{2}+y^{2}}}{r_{\mathrm{e}, \mathrm{CO}}}\right] \\
& \times \exp \left[-4 \ln (2)\left(\frac{x^{2}}{\theta^{2}}+\frac{(y \cos (i))^{2}}{\theta^{2}}\right)\right] \mathrm{d} x \mathrm{~d} y .
\end{aligned}
$$

The inclination $i$ of the galaxy is also accounted for in Eq. (9). It may not be clear at first sight that the effect of $i$ can be written within the Gaussian term, because in principle it should be taken into account in the source and not in the beam. This can be understood when bringing to mind that the integration interval $\mathrm{d} y$ on the sky corresponds to a larger space interval on the source. This means that in the case of accounting for inclination in the source term, also the integration interval had to be changed to $\mathrm{d} y^{\prime}=\mathrm{d} y / \cos (i)$, where $\mathrm{d} y^{\prime}$ is the interval on the source. If one takes into account this effect, the result is the same as in the given formula, meaning that Eq. (9) can be derived via variable substitution from $y$ to $y^{\prime}=y \cos (i)$. Since this cannot be solved analytically, we carry out a numerical integration to find the aperture correction factor $C_{\mathrm{AP}}$ :

$C_{\mathrm{AP}}=\frac{I_{\mathrm{CO}, \text { extra }}}{I_{\mathrm{CO}, \mathrm{obs}}}$.

For LARS galaxies with $\mathrm{CO}(1-0)$ detection, $C_{\mathrm{AP}}$ ranges from 1.01-1.94, with an average correction of $30 \%$. When calculating $C_{\mathrm{AP}}$, the peak brightness temperature $I_{0}$ is cancelled out, but the scale length of the $\mathrm{CO}$ distribution, $r_{\mathrm{e}, \mathrm{CO}}$, is needed as an input. Previous studies showed that $r_{\mathrm{e}, \mathrm{CO}}$ can be estimated from the optical, blue $D_{25}$ diameter (Young et al. 1995; Leroy et al. 2008; Lisenfeld et al. 2011; Davis et al. 2013), roughly independent of the Hubble type:

$r_{\mathrm{e}, \mathrm{CO}}=a \times 1 / 2 D_{25}$,

with a typically ranging from 0.2 to 0.25 . For our work, we choose a value in-between, $a=0.23$. The inclination of the galaxy is calculated using the relation of Hubble (1926),

$\cos ^{2}(i)=\frac{\left(\frac{b}{a}\right)^{2}-q^{2}}{1-q^{2}}$.

We adopt $q=0.2$, and minor-to-major axis ratios, $b / a_{\mathrm{M} 18}$, previously derived by Micheva et al. (2018) (see Table 1).

\subsubsection{Metallicity-dependent $\mathrm{CO}-$ to- $\mathrm{H}_{2}$ conversion}

The CO-to- $\mathrm{H}_{2}$ conversion factor, $\alpha_{\mathrm{CO}}$, is a (virialized) mass-tolight ratio in units of $\left(\mathrm{K} \mathrm{km} \mathrm{s}^{-1} \mathrm{pc}^{2}\right)^{-1}$ that is used to translate $L_{\mathrm{CO}}^{\prime}$ into the molecular mass (including Helium). The extreme conditions in the ISM of (some) LARS galaxies, will potentially cause large uncertainties in $\alpha_{\mathrm{CO}}$, for example due to cloud-cloud collisions. Bolatto et al. (2013) provide a review of the topic. However, the strongest effect on $\alpha_{\mathrm{CO}}$ variations may emerge due to low metallicity, because at some (low $Z$ ) point $\mathrm{CO}$ is not able to shield itself from the ambient radiation field, and is photodissociated, while $\mathrm{H}_{2}$ survives at much larger column densities. Thus, the fraction of so-called CO-dark molecular gas increases, an effect that needs to be accounted for by $\alpha_{\mathrm{CO}}$.

A variety of prescriptions for the metallicity dependence of $\alpha_{\mathrm{CO}}$ exist in the literature (Magdis et al. 2011; Genzel et al. 2012; Narayanan et al. 2012; Schruba et al. 2012; Hunt et al. 2015; Amorín et al. 2016; Accurso et al. 2017). We decided to make use of a semi-analytic relation between metallicity $Z^{\prime}$, the $\mathrm{O} / \mathrm{H}$ abundance in proportion to the solar abundance, and the conversion factor $\alpha_{\mathrm{CO}}$, published by Narayanan et al. (2012):

$\alpha_{\mathrm{CO}}=\frac{\min \left[6.3,10.7 \times I_{\mathrm{CO}}^{-0.32}\right]}{Z^{\prime 0.65}}$.

We discuss the reasoning behind our choice in Appendix E. For the abundances of LARS galaxies given in Table 1, a solar reference value of $[12+\log (\mathrm{O} / \mathrm{H})=8.7]$ was chosen.

\subsubsection{Upper limit molecular masses from $\mathrm{CO}(1-0)$}

For the six LARS galaxies that remained undetected in $\mathrm{CO}(1-$ 0 ), we calculate upper limit CO luminosities from the two-sigma baseline rms (measured outside the line window) multiplied by the square root of the number of channels found within a (typical) $200 \mathrm{~km} \mathrm{~s}^{-1}$ wide line window. The derived $\mathrm{CO}$ upper limit luminosity is then further multiplied by the aperture correction factor and metallicity-dependent conversion factor $\alpha_{\mathrm{CO}}$ exactly as described above. However, we caution that the aperture correction is strictly only valid for peaked $\mathrm{CO}$ distributions with an exponential radial decline. For sources with more complex morphology - and this is the case for a few of our galaxies beam dilution might lower the perceived signal in an unforeseeable way. Moreover, the relatively low metallicity of the galaxies leads to hardly predictable beam filling factors, due to large fractions of CO-dark gas (Schruba et al. 2017), which further reduces the ability to accurately derive limits. The reported upper limits have thus to be taken with caution. 


\subsection{Star formation rates from SED Models, $22 \mu \mathrm{m}$, [C II] $158 \mu \mathrm{m}$, and [O I]63 $\mu \mathrm{m}$}

Star formation rates can be derived through many different methods and tracers (see Kennicutt \& Evans 2012, for a review on the topic). In what follows, unless otherwise noted, we calculate SFRs from the total infrared luminosity $L_{\mathrm{TIR}}$ that we find from the integration of our best-fit dust model SEDs over a range of 3-1100 $\mu \mathrm{m}$. Using the prescription in Kennicutt \& Evans (2012, Table 1 therein), which is based on work by Hao et al. (2011) and Murphy et al. (2011), we translate $L_{\mathrm{TIR}}$ into SFR, assuming a Kroupa \& Weidner (2003) initial mass function (IMF). Consequently, whenever comparing any of our results to values in the literature, we pay attention to the underlying IMF and make adoptions, if needed. Thus, we convert from a Salpeter (1955) to a Kroupa IMF using a constant factor of 0.66. Given the similarity between Kroupa and Chabrier (2003) IMFs, no changes are made in that case.

Next, we briefly compare and discuss a few other prescriptions to estimate star formation rates: an extrapolation from $22 \mu \mathrm{m}$ single-band observations (Rieke et al. 2009), estimates from extinction-corrected $\mathrm{H} \alpha$, and SFRs from [C II] $158 \mu \mathrm{m}$ and [O I]63 $\mu \mathrm{m}$ emission lines (using the prescriptions of De Looze et al. 2014). Taking the far-infrared SED-based SFRs as a reference, we find that both the $22 \mu \mathrm{m}$ photometry and the [O I] $63 \mu \mathrm{m}$ line agree very well, with only $\sim 10 \%$ differences on average (see Table 9).

The situation is worse for (extinction-corrected) $\mathrm{H} \alpha$ and [C II], which lead to SFRs that are (on average) higher by factors of approximately three and approximately five compared to the infrared based estimates. However, for the case of [C II], the discrepancy is mainly driven by LARS 5, a galaxy that is known to have a large scale outflow (Duval et al. 2016). Our finding is thus in agreement with other studies (Appleton et al. 2013, 2018; Smirnova-Pinchukova et al. 2019) that reported on excess [C II $] 158 \mu \mathrm{m}$ emission due to shocks connected to out flowing gas. Excluding LARS 5, the [C II] prescription of De Looze et al. (2014) leads to SFRs that are only $\sim 80 \%$ higher. It is further recognised that SFRs from (extinction-corrected) $\mathrm{H} \alpha$ are comparable to those from [C II], at least once LARS 5 is excluded. In that case the ratio between SFRs from [C II] and $\mathrm{H} \alpha$ is 1.7 only.

The reason for the elevated SFRs from $\mathrm{H} \alpha$ and [C II] compared to the IR based results, is likely due to the sensitivity of the tracers to different timescales. Both $\mathrm{H} \alpha$ and [C II] $158 \mu \mathrm{m}$ are sensitive to the currently ongoing star formation on timescales of $\sim 10 \mathrm{Myr}$, because they are directly linked to the recombination within HII regions that is driven by young, massive stars. On the other side, for the continuum at infrared wavelengths and the TIR emission, a significant fraction of the heating is attributed to more evolved stars. Hence, the timescale probed by TIR is longer ( $\sim 100 \mathrm{Myr})$. The aforementioned discrepancy between the SFRs might thus be caused by varying star formation histories (Melinder et al., in prep.), given the relatively young age of LARS galaxies, due to their being selected as having $\mathrm{H} \alpha$ equivalent widths of more than $100 \AA$ (Östlin et al. 2014).

\subsection{SFR and gas surface densities}

For calculations of star formation rate surface densities, $\Sigma_{\mathrm{SFR}}$, and gas surface densities, $\Sigma_{\text {gas }}$, we normalize the measured integrated values to the area enclosed by the $25 \mathrm{mag} \mathrm{arcsec}^{-2}$ isophote in the optical $B$-band, $D_{25_{\text {sDss }}}=\pi \times a / 2 \times b / 2$. The diameter $D_{25_{\text {sDss }}}$ was calculated from the Sloan Digital Sky Survey
Table 9. Comparison of SFR estimates for LARS galaxies.

\begin{tabular}{lrrrrr}
\hline \hline ID & SFR $_{\mathrm{H} \alpha}$ & $\mathrm{SFR}_{22 \mu \mathrm{m}}$ & $\begin{array}{r}\mathrm{SFR}_{[\mathrm{CII}]} \\
(1)\end{array}$ & $\begin{array}{r}\mathrm{SFR}_{[\mathrm{OI}]} \\
(2)\end{array}$ & $\begin{array}{r}\mathrm{SFR}_{\mathrm{TIR}} \\
(6)\end{array}$ \\
\hline 1 & 6.5 & $4.6 \pm 0.12$ & - & - & $2.13_{-0.56}^{+1.66}$ \\
2 & 1.4 & $0.2 \pm 0.06$ & $0.4 \pm 0.2$ & $<2.3$ & $0.28_{-0.11}^{+0.23}$ \\
3 & 26.3 & $105.7 \pm 0.3$ & $35.5 \pm 0.8$ & $73 \pm 3$ & $65.76_{-2.48}^{+17.41}$ \\
4 & 6.1 & $5.2 \pm 0.15$ & - & - & $4.00_{-0.18}^{+0.47}$ \\
5 & 5.8 & $2.0 \pm 0.1$ & $29 \pm 5$ & - & $0.81_{-0.17}^{+0.41}$ \\
6 & 0.6 & - & - & - & $0.27_{-0.06}^{+0.18}$ \\
7 & 9.3 & $4.6 \pm 0.2$ & - & - & $2.99_{-0.15}^{+0.74}$ \\
8 & 36.8 & $31.0 \pm 0.32$ & $81 \pm 13$ & - & $29.02_{-6.38}^{+9.25}$ \\
9 & 40.7 & $24.7 \pm 0.44$ & $33.4 \pm 0.6$ & $34 \pm 3$ & $25.31_{-3.56}^{+0.40}$ \\
10 & 5.6 & $1.9 \pm 0.3$ & - & - & $6.69_{-2.22}^{+0.93}$ \\
11 & 30.5 & $28.4 \pm 1.01$ & $203 \pm 12$ & - & $38.78_{-5.96}^{+14.54}$ \\
12 & 97.0 & $12.7 \pm 0.72$ & $8.7 \pm 1.2$ & $16 \pm 4$ & $9.99_{-0.12}^{+0.88}$ \\
13 & 64.8 & $66.5 \pm 3.02$ & $40.5 \pm 4.1$ & $54 \pm 10$ & $106.71_{-10.07}^{+6.35}$ \\
14 & 24.8 & $7.0 \pm 2.13$ & - & - & $5.79_{-0.14}^{+0.98}$ \\
\hline
\end{tabular}

Notes. The SFRs are given in units of $M_{\odot} \mathrm{yr}^{-1}$. The LARS ID is shown in Col. 1. Previously published SFRs from extinction-corrected $\mathrm{H} \alpha$ (Hayes et al. 2014) are found in Col. 2. New SFR estimates from WISE $22 \mu \mathrm{m},[\mathrm{C} \mathrm{II}] 158 \mu \mathrm{m}$, [O I] $63 \mu \mathrm{m}$, and total infrared luminosities are given in Cols. 3-6. Total infrared luminosities in Col. 6 are used for all analysis (KS plots etc.) in this paper.

(SDSS) $g$-band, which typically gives sizes that are $\sim 1.3$ times larger than those measured in the Johnson $B$ band (Hakobyan et al. 2012). Thus, we divided the $g$-band diameters by that factor first.

\section{Results}

In this section, scaling relations for LARS galaxies are shown and numerous other quantities such as gas masses, gas fractions, and gas depletion times are tabulated. A relation between the gas depletion time and $\operatorname{Ly} \alpha$ escape fraction is found. We further report on an extreme [C II] $158 \mu \mathrm{m}$ line strength in LARS 5 and perform basic PDR analysis.

\subsection{Scaling of gas mass and SFR}

The scaling relation between dust-traced total gas mass and star formation rate in Fig. 7 shows that LARS galaxies span a range that is more than two orders of magnitude wide in both quantities. LARS comprises the whole parameter space of the COLDGASS survey (Saintonge et al. 2011a,b, 2012; Catinella et al. 2012), a representative sample of $z \sim 0$ galaxies with masses above $10^{10} M_{\odot}$. A comparison between the two applied methods to calculate gas masses (dust-based versus $\mathrm{CO}+\mathrm{HI}$ based) is shown in Fig. 8. For those galaxies (LARS 3, 8, 9, 11) with combined measurements of $\mathrm{CO}+\mathrm{HI}$ available (LARS 13 was not detected in H I previously), we find an excellent match between the two methods; on average they agree on a level better than $\sim 0.2$ dex. The upper limit molecular masses from our $\mathrm{CO}(1-0)$ observations may be affected by beam dilution, leading to an increase in the limits towards higher masses. We only use dust-based gas mass estimates for the following analysis. 


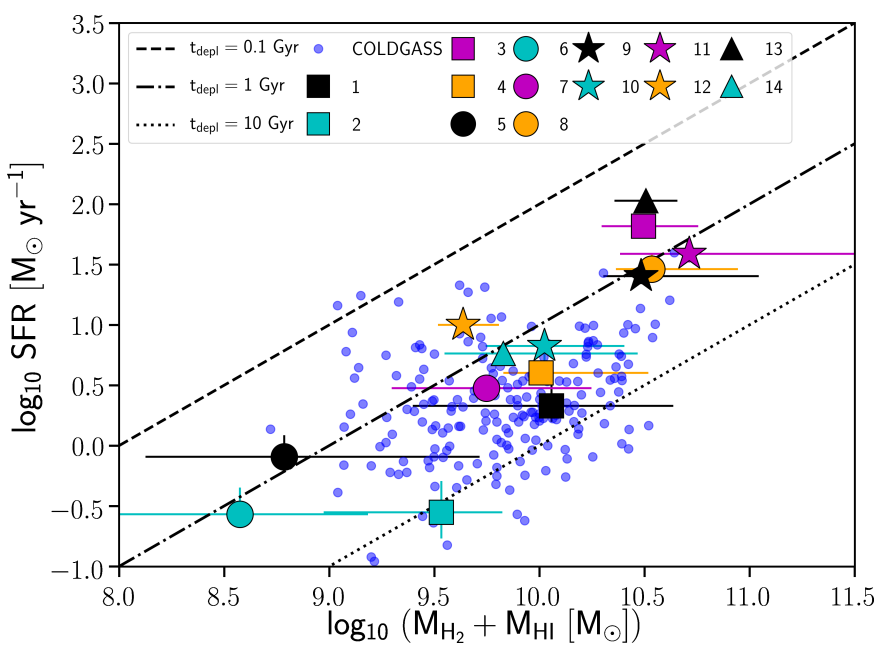

Fig. 7. Gas mass and SFR (from total integrated infrared luminosity) scaling for LARS galaxies and COLDGASS, a $z \sim 0$ representative sample of galaxies.

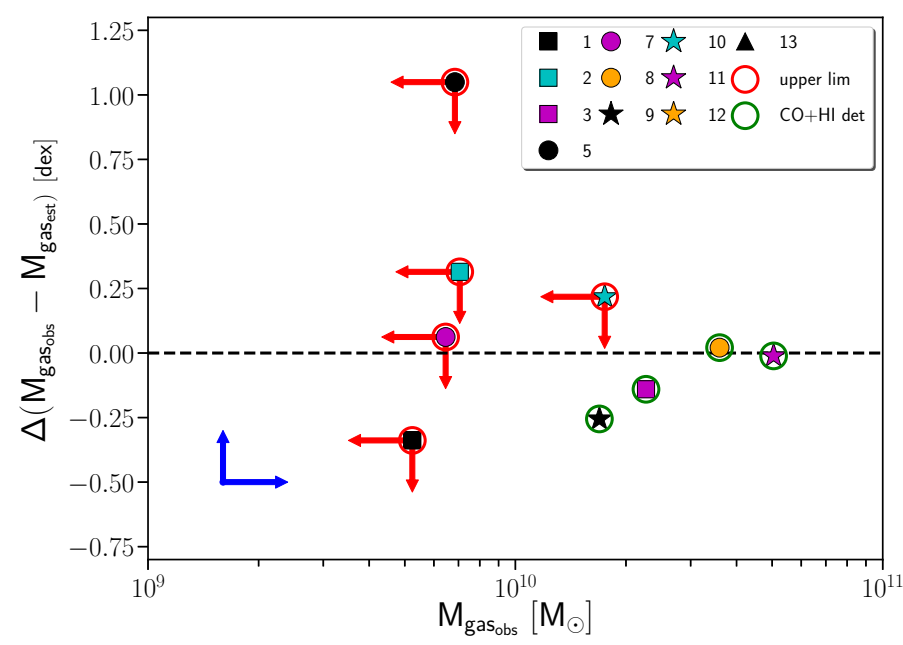

Fig. 8. Total gas masses from combined $\mathrm{CO}(1-0)$ and $\mathrm{HI}$ observations vs. the difference between observed total gas mass $(\mathrm{CO}+\mathrm{HI})$ and the dust-based total gas mass. Galaxies with detection in $\mathrm{CO}+\mathrm{HI}$ are circled in green, while measurements with $\mathrm{CO}$ and/or $\mathrm{H} \mathrm{I}$ upper limits are shown in red. The $\mathrm{CO}$ upper limits may be affected by beam dilution that works in the direction of the blue arrows.

\subsection{KS relation, gas fractions, and depletion times}

The global molecular Kennicutt-Schmidt (KS) relation for LARS is shown in the top panel of Fig. 9. We compare our sample to the COLDGASS population as well as to $z \sim 1-3$ massive star-forming main sequence galaxies of Genzel et al. (2010). Compared to COLDGASS galaxies our sample is biased towards higher $\mathrm{H}_{2}$ surface densities, with LARS 9 at the extreme end, where typically only high- $z$ star-forming galaxies are located. At the lowest end, LARS 2 is found. The molecular gas depletion time, defined as the ratio between $\Sigma_{M_{\mathrm{H}_{2}}}$ and $\Sigma_{\mathrm{SFR}}$, seems to be rather consistent for the whole sample, with a scatter of only $\pm 1 \mathrm{Gyr}$ around the median value of $0.7 \mathrm{Gyr}$. The classical KS relation in the bottom panel of Fig. 9 is slightly different. The total gas depletion time, which is the ratio between $\Sigma_{M_{\mathrm{H}_{2}}+M_{\mathrm{HI}}}$ and $\Sigma_{\mathrm{SFR}}$, has a scatter that is three times larger $( \pm 3 \mathrm{Gyr})$ around the median of $1.3 \mathrm{Gyr}$ for the whole sample.
The inverse of the gas depletion time is often denoted as the star formation efficiency (SFE), which is the star formation rate per unit gas mass. However, it is important to understand that the SFE as defined above is not necessarily an indication of a true efficiency or ability to form stars per unit gas mass, because not all the observed gas is readily available to fuel the current star formation. The efficiency is thus degenerate with the gas fraction. For that reason, Krumholz et al. (2012) suggest the SFE should be normalized by the cloud-scale gravitational free-fall time $\tau_{\mathrm{ff}}$. This decreases the scatter in the classical KS law. Unfortunately, $\tau_{\mathrm{ff}}$ cannot be assessed from our observations because the determination of $\tau_{\mathrm{ff}}$ involves knowledge of the volume density of the gas, a quantity that cannot be performed based on our observations. For that reason we mainly make use of the inverse of the SFE as defined above, which is the gas depletion time $\left(\tau_{\text {gas }}\right)$, that is, the time span over which star formation may be maintained at the current level given the amount of gas available.

With previously published stellar masses (Hayes et al. 2014) that are given in Col. 3 of Table 1, the total gas fraction can be defined: $f_{\text {gas }}=M_{\text {gas }_{\text {est }}} /\left(M *+M_{\mathrm{gas}_{\mathrm{est}}}\right)$. For LARS the total gas fractions vary from $\sim 15-80 \%$. All derived quantities discussed in this section are summarized in Table 10.

\subsection{Compactness and characteristic size of star formation activity}

Figure 10 shows the star formation rate versus the surface density of star formation, $\Sigma_{\mathrm{SFR}}^{\mathrm{rcut}}$; in this case the SFR is normalized to the area of star-forming activity (derived by Micheva et al. 2018 from FUV imaging) rather than stellar content. It is striking that compared to the previous $\mathrm{KS}$ plots the scatter in the $\mathrm{y}$ direction is largely decreased. It is also striking that compared to other galaxy samples, the size of the star-forming activity in LARS galaxies is found to be roughly constant with a median diameter of $\sim 1 \mathrm{kpc}$, similar in size to circum-nuclear star formation activity. However, a weak but tight trend is seen between $\Sigma_{\text {SFR }}^{\text {rcut }}$ and SFR, in a sense that galaxies with high star formation rates exhibit larger and more crowded areas of star formation. A possible explanation for the tighter spread seen in $\Sigma_{\text {SFR }}^{\text {rcut }}$ compared to $\Sigma_{\mathrm{SFR}}$ may be found in the star formation history, which varies from one LARS galaxy to another and has led to varying fractions and sizes of a (older) stellar populations, while the currently ongoing star formation density is similar.

\subsection{Grouping LARS according to $\tau_{\text {gas }}$}

An examination of the gas depletion times and gas fractions (see Table 10) reveals that the heterogeneity of LARS galaxies that is seen in the KS law is related to $\operatorname{Ly} \alpha$ escape. While no trend is seen between total gas surface density and Ly $\alpha$ escape, we find a strong trend between the total gas depletion time and the Ly $\alpha$ escape fraction (see Fig. 11). This is also seen when grouping LARS galaxies into bins of increasing total gas depletion times $\left(\tau_{\text {gas }}\right)$ :

- Group 1 ( $\tau_{\text {gas }} \lesssim 1$ Gyr): As seen in Fig. 9, the galaxies with the shortest gas depletion times are LARS 3, 5, 12, and 13 .

- Group 2 ( $\left.1 \mathrm{Gyr} \lesssim \tau_{\text {gas }} \lesssim 2 \mathrm{Gyr}\right)$ : In the group of intermediate gas depletion times - with values that are typically found in main sequence galaxies - we find LARS 6, 8, 9, 10, 11, and 14 . This group is the largest of the three and the galaxies within it are quite diverse. In fact we could break up the group into high and low stellar mass sub-samples. While the high-mass galaxies (LARS 8, 9, 10, and 11) might indeed be similar to $z \sim 1-2$ main 

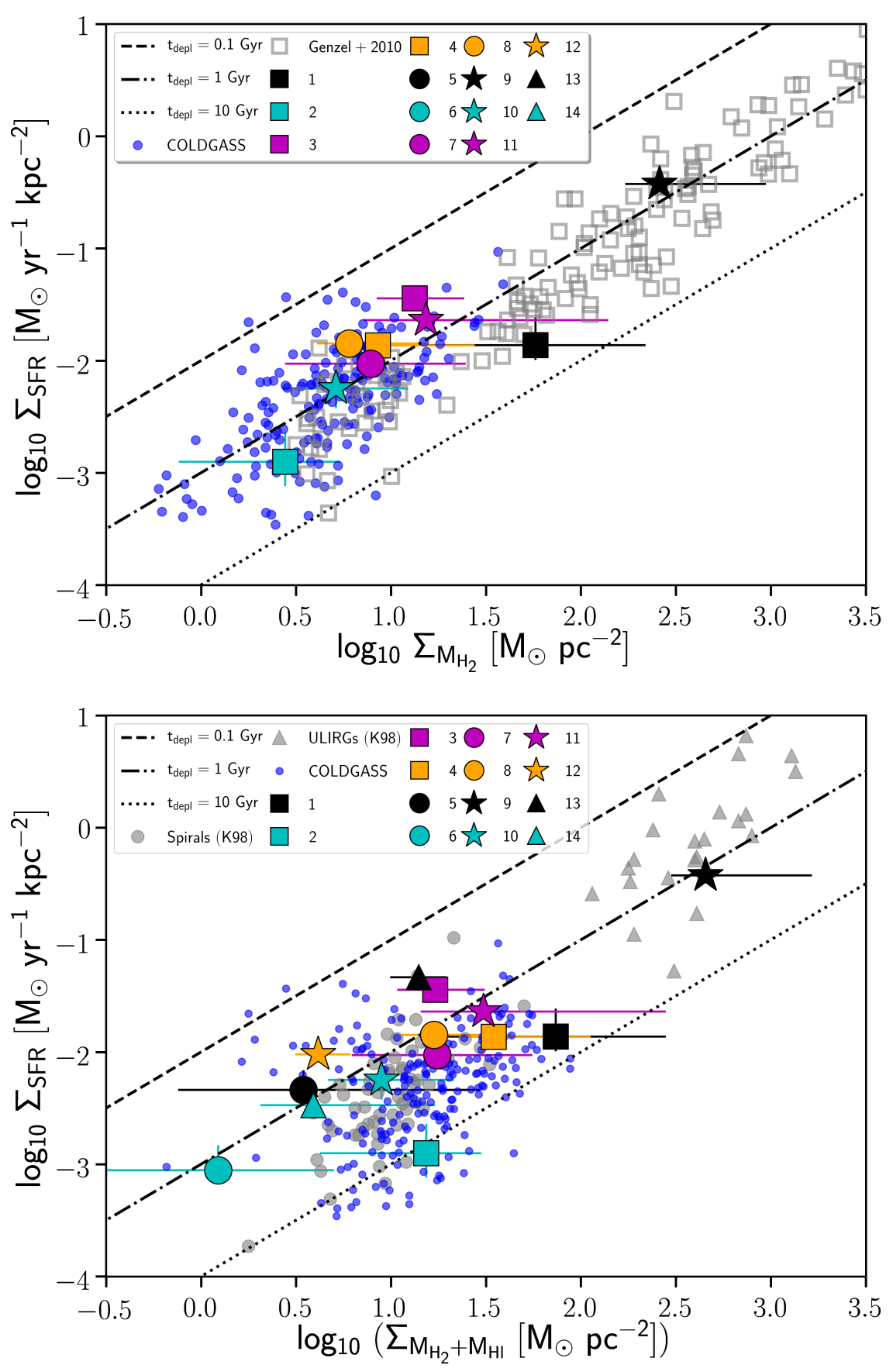

Fig. 9. Top panel: molecular $\mathrm{KS}$ relation: $\mathrm{H}_{2}$ surface density (from dust-based gas mass minus atomic mass) vs. star formation rate surface density (from total integrated $L_{\mathrm{TIR}}$ ) for LARS compared to COLDGASS (blue) and a sample of $z \sim 1-3$ star-forming galaxies from Genzel et al. (2010) (grey open squares). Bottom panel: KS relation: total gas surface density (dust-based) vs. star formation rate surface density (from total integrated $L_{\mathrm{TIR}}$ ). The COLDGASS galaxies (blue) and Kennicutt (1998) samples of local spirals (grey filled circles) and ultra-luminous infrared galaxies (grey filled triangles) are shown as reference. Black diagonal lines indicate (from top to bottom) constant gas depletion times of $0.1,1$ and $10 \mathrm{Gyr}$. sequence galaxies, a characterization of the low-mass galaxies $(6,14)$ is difficult.

- Group 3 ( $\tau_{\text {gas }} \gtrsim 2$ Gyr): Those LARS galaxies that have relatively high gas surface densities and low SFR surface densities $(1,2,4,7)$ have much larger total gas fractions.

Next, we calculate for each $\tau_{\text {gas }}$ group the average and median physical quantities that we previously derived and relate those to the properties of $\operatorname{Ly} \alpha$ escape. The result is shown in Table 11. We note that the results hold-up when the median is used instead of the average. It is noteworthy that LARS galaxies with the longest depletion times (Group 3) also exhibit the highest Ly $\alpha$ escape fractions (more than double the average in the sample), while they have the shortest Ly $\alpha$ scattering distances. Moreover their average environment and ISM conditions are significantly different from the other two groups, in a sense that their mean (FUV) energy density $U_{\text {min }}$ is the lowest and their total gas fractions are the highest (while having the lowest molecular gas fraction). We discuss this further in Sects. 5.1 and 5.2.

\subsection{Extreme [C II]158 $\mu \mathrm{m}$ line strength in LARS 5 and basic PDR analysis}

In eight LARS galaxies, the [C II] $158 \mu \mathrm{m}$ line was successfully detected, either with Herschel/PACS or SOFIA/FIFI-LS. We first examine the relative strength of the [C II] $158 \mu \mathrm{m}$ line compared to the total far-infrared energy. As seen in Fig. 12, LARS 5 is an extreme outlier in the plot. Neither low-ionization nuclear emission-line region (LINER) galaxies, Seyfert, or quasars from SHINING, the survey with Herschel of thes interstellars medium 
Table 10. Derived properties for LARS galaxies.

\begin{tabular}{|c|c|c|c|c|c|c|c|c|c|c|c|c|c|c|c|c|}
\hline ID & $\begin{array}{c}\log _{10} \\
\Sigma_{\text {SFR }} \\
(2) \\
\end{array}$ & $\begin{array}{c}\log _{10} \\
\Sigma_{\text {SFR }}^{\text {rcut }} \\
(3) \\
\end{array}$ & GDR & $\alpha_{\mathrm{CO}}$ & $C_{\mathrm{AP}}$ & $\begin{array}{c}\log _{10} \\
L_{\mathrm{CO}}^{\prime} \\
(7) \\
\end{array}$ & $\begin{array}{c}\log _{10} \\
M_{\mathrm{H}_{2}} \\
\quad(8)\end{array}$ & $\begin{array}{c}\log _{10} \\
M_{\mathrm{H}_{2}} \\
(9)\end{array}$ & $\begin{array}{c}\log _{10} \\
M_{\mathrm{gas}_{\mathrm{obs}}} \\
(10)\end{array}$ & $\begin{array}{c}\log _{10} \\
M_{\text {gas }_{\text {est }}} \\
(11)\end{array}$ & $\begin{array}{l}\log _{10} \\
\Sigma_{\text {gas }} \\
(12) \\
\end{array}$ & $\begin{array}{c}\tau_{\text {gas }} \\
\times 10^{8} \\
(13) \\
\end{array}$ & & & $f_{\mathrm{mol}}$ & $\mathrm{DR}$ \\
\hline 1 & $86 t$ & .27 & 455.0 & 11.40 & 1.07 & 4 & $<94$ & 16 & $<9.719$ & .058 & 1.868 & 53.70 & 065 & 022 & 0.57 & 21 \\
\hline 2 & - & $.0^{4}$ & & 10 & & $<$ & & & & & & 12 & & & & 1 \\
\hline 3 & -1.444 & 1.722 & 06. & 5.10 & 1.9 & 9.549 & 10.198 & 10.2 & 10.35 & 96 & 4 & 4.8 & & & & 16 \\
\hline 4 & -1.866 & 0.34 & 11. & - & - & - & - & 9.054 & - & 10.009 & 1.541 & 25.50 & 0.44 & 0. & 0.11 & 13 \\
\hline 5 & -2.339 & -0.523 & 597. & 14.80 & 1.06 & 8.48 & $<9.596$ & - & $<9.83$ & 786 & 0.539 & 7.5 & 0.13 & 4.75 & - & .08 \\
\hline 6 & -3.053 & -0.82 & 99 & 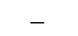 & - & - & 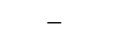 & - & - & 0. & 0 & 13. & 0.1 & 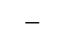 & - & .01 \\
\hline 7 & -2.029 & 0.10 & 353 & 11.0 & 1.10 & 8.5 & $<9.52$ & 9.22 & $<9$. & 9.7 & 14 & 18. & 0.54 & 0. & 0 & 14 \\
\hline 8 & -1.8 & 0.152 & 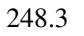 & & 1.3 & 9.54 & 10.1 & 10.030 & 10. & 0.535 & 1.226 & 11. & 0.2 & 0. & 1 & .05 \\
\hline 9 & -0.4 & 0.15 & 342.0 & 1 & 1 & & 9.5 & 10.173 & 10.2 & $t$ & 6 & 12 & 7 & 3 & 9 & 09 \\
\hline 10 & -2.2 & 0.164 & 248.3 & 6.30 & 1. & 9.3 & 10. & 9. & $<10$ & 10 & 0 & 15.8 & 3 & 2 & 7 & 00 \\
\hline 11 & - & & 291 & & & & & 10.377 & 10.70 & 10 & & 1 & & 0.50 & 0.46 & 04 \\
\hline 12 & -2.021 & -0.135 & 61.4 & 10.20 & 1.03 & $<9.4$ & $<10.465$ & - & - & & 17 & 4.3 & 0. & 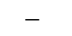 & - & 0.11 \\
\hline 13 & -1.333 & & 249.5 & 11.30 & 1.03 & 9.273 & 10.364 & - & - & 10. & & 3.0 & $0 .$. & - & & 0.08 \\
\hline 14 & -2.474 & -0.536 & 848.2 & - & - & - & - & - & - & 9.829 & 0.592 & 11.60 & 0.79 & - & - & 0.21 \\
\hline
\end{tabular}

Notes. Surface densities in Cols. (2) and (10) are normalized to the $B$-band $25 \mathrm{mag} \operatorname{arcsec}^{-2}$ area, while the SFR surface density in Col. (3) is normalized to the star-forming area only. The $\mathrm{CO}$ luminosity in Col. (7) was derived from the $\mathrm{CO}$ velocity-integrated main beam brightness temperature and subsequent multiplication with the aperture correction factor in Col. (6). For galaxies with CO detection, $M_{\mathrm{H}_{2}}$ in $\mathrm{Col}$. (8) was calculated from the CO luminosity in Col. (7) via application of the conversion factor in Col. (5). For galaxies with positive CO and $\mathrm{HI}$ detection, $M_{\mathrm{gas}_{\mathrm{obs}}}$ is the sum of the atomic mass from Pardy et al. (2014) and the molecular mass in Col. (8). Total gas masses $M_{\text {gasest }_{\text {est }}}$ in Col. (11) were derived from the dust masses using a metallicity-dependent gas-to-dust ratio shown in Col. (4). The estimated molecular mass $M_{\mathrm{H}_{2 \text { ect }}}$ is the result of the dust-based total mass $M_{\mathrm{gas}_{\mathrm{est}}}$ minus the observed atomic gas mass. The total gas surface density in Col. (12), the total gas depletion time $\tau_{\text {gas }}$ in Col. (13), as well as the total gas fraction $f_{\text {gas }}=M_{\mathrm{gasest}_{\mathrm{es}}} /\left(M *+M_{\mathrm{gasest}_{\mathrm{es}}}\right)$ in Col. (14) use the dust-based total gas mass estimate. The atomic gas fraction $f_{\mathrm{HI}}$ is the ratio between the observed $\mathrm{HI}$ gas mass and the total dust-based gas mass. The molecular gas fraction $f_{\text {mol }}$ is the ratio between the estimated molecular gas mass and the total dust-based gas mass. The PDR gas mass fractions in Col. (17) are the $\gamma$ values from Table 8 on a linear scale. We note that the large discrepancies between the reported upper limits in Cols. (8) and (10) and the dust-based estimated quantities are likely the result of beam dilution, that is, the upper limits are likely misleading. The units of the derived quantities are: (2), (3): $M_{\odot} \mathrm{yr}^{-1} \mathrm{kpc}^{-2}$ (5): $\left(\mathrm{K} \mathrm{km} \mathrm{s}^{-1} \mathrm{pc}^{2}\right)^{-1}(7): \mathrm{K} \mathrm{km} \mathrm{s}^{-1} \mathrm{pc}^{2}(8)-(11): M_{\odot}(12): M_{\odot} \mathrm{pc}^{-2}(13): \mathrm{yr}$.

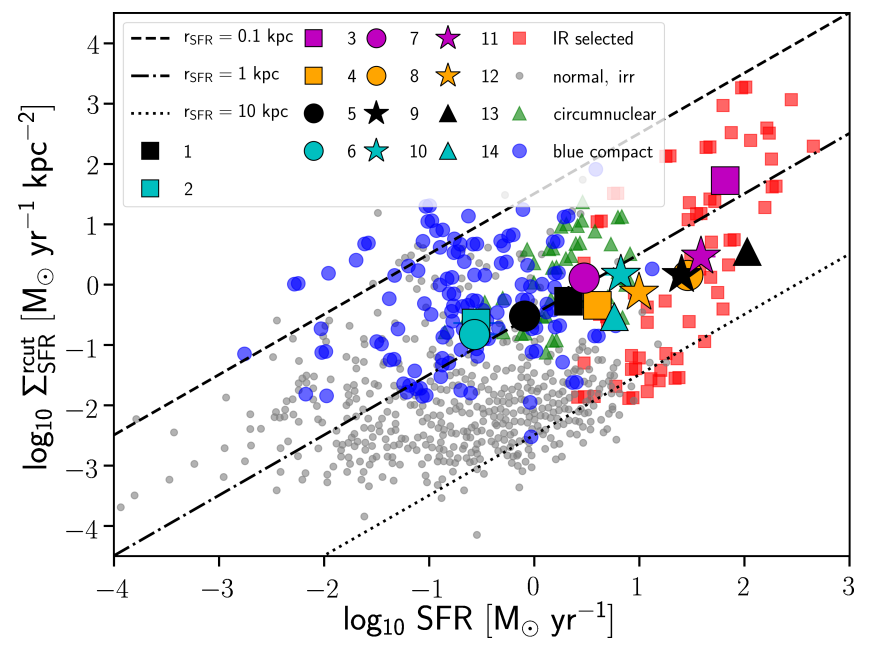

Fig. 10. Total SFR versus the star formation rate density (both using SED integrated $L_{\mathrm{TIR}}$ as tracer) normalized by the area of activity (as seen in UV). Over-plotted are comparison samples from Kennicutt \& Evans (2012).

in nearby infrared galaxies (Herrera-Camus et al. 2018a,b; Zhao et al. 2016), nor any other non-active galactic nucleus galaxy observed to date (see figure caption for details of the comparison samples), has a global [C II] $158 \mu \mathrm{m}$ to FIR ratio on the order of $\sim 14 \pm 3$ percent as seen in LARS 5 (see also Table 12). The mechanism that drives the extreme ratio is likely connected to the large scale galactic wind that was previously reported and studied by Duval et al. (2016). This is supported by the fact that

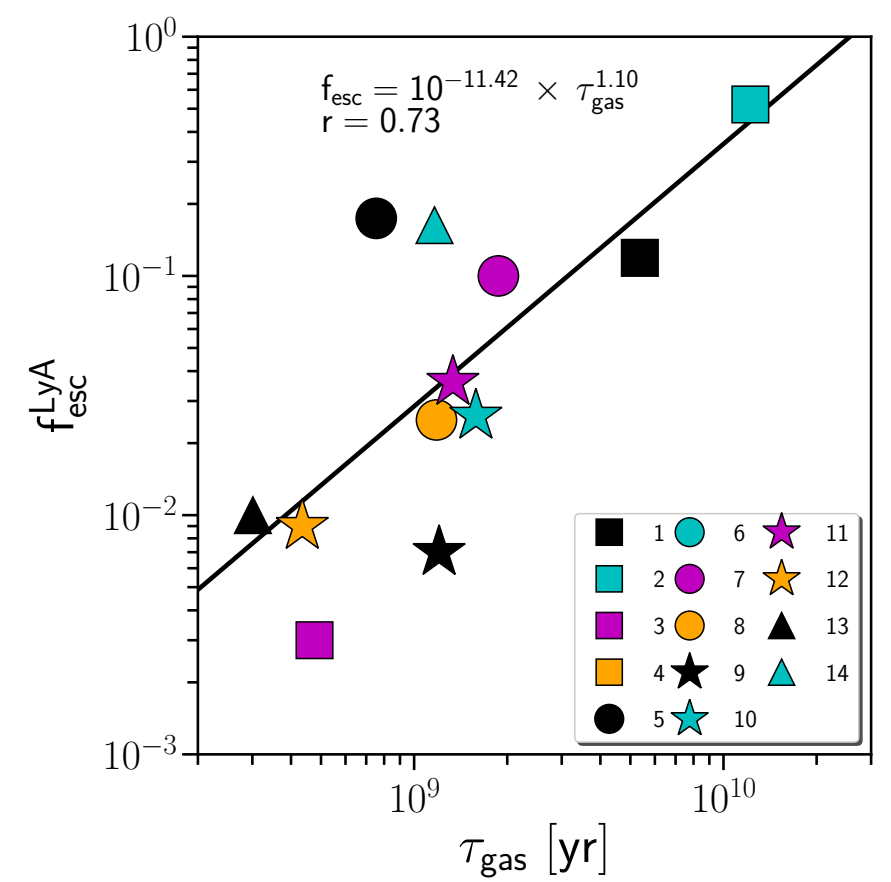

Fig. 11. Relation between total gas depletion time and Ly $\alpha$ escape fraction. LARS 4 and 6 have a global Ly $\alpha$ escape fraction of zero and are thus omitted from the fit.

both the [C II] $158 \mu \mathrm{m}$ and [O III] $88 \mu \mathrm{m}$ emission lines are blueshifted from the systemic velocity, as seen in Figs. 4 and 6. We note that shock-enhanced [C II] $158 \mu \mathrm{m}$ excess was previously 
observed in other galaxies with outflows (Appleton et al. 2013, 2018; Smirnova-Pinchukova et al. 2019).

While LARS 5 is an extreme outlier, most of the other galaxies detected in [C II] $158 \mu \mathrm{m}(3,8,9,11,12$, and 13) also lie on the upper end of what is typically observed in local galaxies. On the other hand, LARS 2 has a much weaker relative [C II] line strength, which is interesting considering the fact that LARS 2 is the only galaxy with a [C II] detection that is member of Group 3 (while LARS 7 remained undetected despite having the longest integration time).

We perform a very basic PDR analysis based on the models of Kaufman et al. (1999, 2006). Using the far infrared luminosity $\left(L_{\mathrm{FIR}_{\mathrm{w}}}\right)$ and emission lines of [C II] $158 \mu \mathrm{m}$ and [O I]63 $\mu \mathrm{m}$, we apply PDR Toolbox (Pound \& Wolfire 2008) to fit the observed line ratios against the model predictions. The results for the bestfit model are summarized in Table 13. The quality of the fits can be assessed from Fig. C.1, where the contours indicate 1, 2, and $3 \sigma$ significance regions that are confined by $\chi_{\alpha}^{2}=\chi_{\min }^{2}+\delta(\nu, \alpha)$ (Wall 1996), with the $\chi^{2}$-difference $\delta$ being a function of degrees of freedom $v$ (in our case $v=1$ ) and $\alpha$ the desired significance (in our case $0.68,0.95$, and 0.99). For LARS 3 and 9, the models are not able to reproduce the observations in which $\chi_{\min }^{2} \gg 1$. This might be related to the fact that both are merging galaxies. However, for the remaining galaxies, LARS 2, 12, and 13 , we find model fits with uncertainties of $\pm 0.6, \pm 0.15$, and \pm 0.1 dex respectively. We can also see in Fig. C. 1 that the solution plane shows two valleys (bi-modality), with density and radiation field strength being degenerate. However, solutions at lower gas densities and stronger radiation field strengths $G_{0}$ are more pronounced. For the second valley the solutions would lead to $G_{0}<3$, making them relatively low given the starburst nature of our galaxies. We caution that we apply models that are valid only for a particular regime (PDR) to unresolved observations that cover a multi-phase ISM. Thus, the power of such an analysis is limited. However, a comparison of the derived PDR gas volume densities between LARS 2 (which is member of Group 3) and LARS 12 and 13 (Group 1) shows that the density in LARS 2 is approximately five times (or 0.7 dex) higher than the average density of LARS 12 and 13. Assuming that the high PDR gas density holds for the whole of Group 3, this might indicate that the bulk of the star-forming regions are still deeply embedded in their birth clouds, which are not yet disrupted due to stellar feedback. We are thus likely witnessing early stages of star formation. This scenario is supported by the low $\Sigma_{\mathrm{SFR}}$, low $U_{\min }$ values, and high total gas fractions found in Group 3.

Assuming that the high observed PDR gas volume density in LARS 2 reflects a high density in the cold, starforming gas, the free-fall time in LARS 2 is approximately three times shorter, suggesting that the scatter in the KS law once normalized to the free-fall time - could indeed be largely reduced, given that LARS 2 is the most extreme outlier in the relation.

We also put the LARS galaxies onto a widely used diagnostic diagram, as shown in the right panel of Fig. 12, and over-plot lines of constant density together with knot points indicating the FUV field strength $G_{0}$. The shown models were calculated for two metallicities, one with solar and the other one with one tenth solar abundances. We further consider only the case for $A_{V}=1$. With this diagram we mainly want to showcase the general sensitivity of the diagnostic lines. While the [C II] $158 \mu \mathrm{m}$ to FIR ratio mainly defines the density, the $\mathrm{CO}(1-0)$ to FIR ratio is most sensitive to the radiation field.

\subsection{Dense gas fractions in LARS 3 and 8}

For the two LARS galaxies with detections in $\mathrm{HCN}(1-0)$, we calculate the line ratios of $\mathrm{HCN}(1-0) / \mathrm{CO}(1-0)$. Given the fact that the critical density of $\mathrm{HCN}(1-0)$ is almost two orders of magnitude higher than for $\mathrm{CO}(1-0)$, the ratio between these two lines is often used as a tracer of the dense gas fraction (Gao \& Solomon 2004b; Bigiel et al. 2016; Jiménez-Donaire et al. 2017). We find line ratios of 0.13 and 0.05 for LARS 3 and LARS 8 respectively. While the latter is similar to values found for example in M51 (Bigiel et al. 2016), the former ratio indicates an extremely high dense gas fraction that is on the upper limit of what is typically found in infrared-luminous starforming galaxies (compare to e.g. Juneau et al. 2009, Fig. 6). The high dense gas fraction in LARS 3 is likely the result of an ongoing merging process that has triggered a nuclear starburst in the galaxy.

\subsection{Derived properties from line ratios of $\mathrm{CO}, \mathrm{HCN}$, and $\mathrm{HCO}^{+}$}

The IRAM 30m telescope is capable of using two heterodyne receivers at the same time. We made use of this feature and observed $\mathrm{CO}(1-0)$ and $\mathrm{CO}(2-1)$ simultaneously. However, given the different beam sizes and thus different beam filling factors, in combination with the complex morphology of our galaxies, one must be cautious when interpreting line ratios between $\mathrm{CO}(2-1)$ and $\mathrm{CO}(1-0)$. On the other hand, observations of the $\mathrm{CO}(3-2)$ line with APEX and $\mathrm{CO}(1-0)$ with IRAM, give similar beam widths. Hence, the ratio between these two lines may give insight into the average conditions of the molecular gas. As shown in Fig. 13, we detected $\mathrm{CO}(3-2)$ in two of our galaxies. We find $\mathrm{CO}(3-2) / \mathrm{CO}(1-$ 0 ) line ratios of 0.5 and 1.0 (on the brightness temperature scale) for LARS 8 and 13 respectively. The former value indicates subthermal excitation, whereas the conditions in LARS 13 are more extreme and the line ratio suggests thermalized gas up to the $\mathrm{CO}$ $J=3$ level. This result is in agreement with our derivations of $U_{\min }$, the average energy density, which was found to be extremely high for LARS 13 and moderate in LARS 8 (see Table 8).

Line radiative transfer modelling for LARS 3 and 8 . Given the variety of emission lines now available (i.e. $\mathrm{CO}(1-0), \mathrm{CO}(2-$ 1), $\mathrm{HCN}(1-0)$, and $\mathrm{HCO}^{+}(1-0)$ for LARS 3 and 8, as well as $\mathrm{CO}(3-2)$ for the latter), we are able to estimate the molecular gas density using novel line radiative transfer models, described in detail in a forthcoming paper by Puschnig et al. (in prep). The first release of the Dense Gas Toolbox (Puschnig 2020) is readily available, either as a stand-alone web application ${ }^{7}$ or source code ${ }^{8}$. The radiative transfer models rely on RADEX (van der Tak et al. 2007), but extend the original code to take into account that molecular emission lines may emerge from a distribution of densities rather than from a single-zone (single-density) medium. This approach is more realistic, in particular for unresolved or low-resolution observations as in our case, and has a strong impact on interpreting line ratios in terms of physical quantities, as shown by Leroy et al. (2017). We adopt a density distribution that is log-normal and derive the mass-weighted mean density of that underlying gas distribution. Making use of the previously derived dust properties, we assume fixed temperatures of 30 and $25 \mathrm{~K}$ for LARS 3 and 8 respectively. A summary of the line ratios used as input for the modelling is shown in Table D.1. This approach further allows us to derive the dense gas fraction, defined

\footnotetext{
http: //www . densegastoolbox. com

8 https://doi.org/10.5281/zenodo. 3686329
} 
Table 11. Average and median properties of LARS galaxies grouped into bins of increasing gas depletion times.

\begin{tabular}{lcccccc}
\hline \hline & $\begin{array}{c}\tau_{\text {gas }} \\
{\left[10^{8} \mathrm{yr}\right]}\end{array}$ & $\begin{array}{c}f_{\text {esc }}^{\text {LyA }} \\
{[\%]}\end{array}$ & $\begin{array}{c}D_{\text {scatt }} \\
{[\mathrm{kpc}]}\end{array}$ & $\begin{array}{c}f_{\text {gas }} \\
{[\%]}\end{array}$ & $\begin{array}{c}f_{\text {mol }} \\
{[\%]}\end{array}$ & $U_{\text {min }}$ \\
\hline Group 1 $(3,5,12,13)$ & $5(5)$ & $4.9(1.0)$ & $1.0(0.7)$ & $37(36)$ & $62(62)$ & $17.0(17.9)$ \\
Group 2 $(6,8,9,10,11,14)$ & $13(13)$ & $5.1(2.6)$ & $0.9(0.5)$ & $37(32)$ & $46(48)$ & $8.7(8.2)$ \\
Group 3 (1,2,4,7) & $\mathbf{5 5 ( 4 0 )}$ & $\mathbf{2 4 . 6 ( 1 1 . 9 )}$ & $\mathbf{0 . 5 ( 0 . 5 )}$ & $\mathbf{5 6}(\mathbf{5 7})$ & $\mathbf{2 9}(\mathbf{2 4})$ & $\mathbf{7 . 9 ( 7 . 9 )}$ \\
All LARS (incl. 6) & $23(13)$ & $9.9(3.1)$ & $0.8(0.5)$ & $42(37)$ & $40(46)$ & $10.8(9.2)$ \\
\hline
\end{tabular}

Notes. Median quantities are given in brackets. Highlighted in bold is the group with the largest escape fraction.

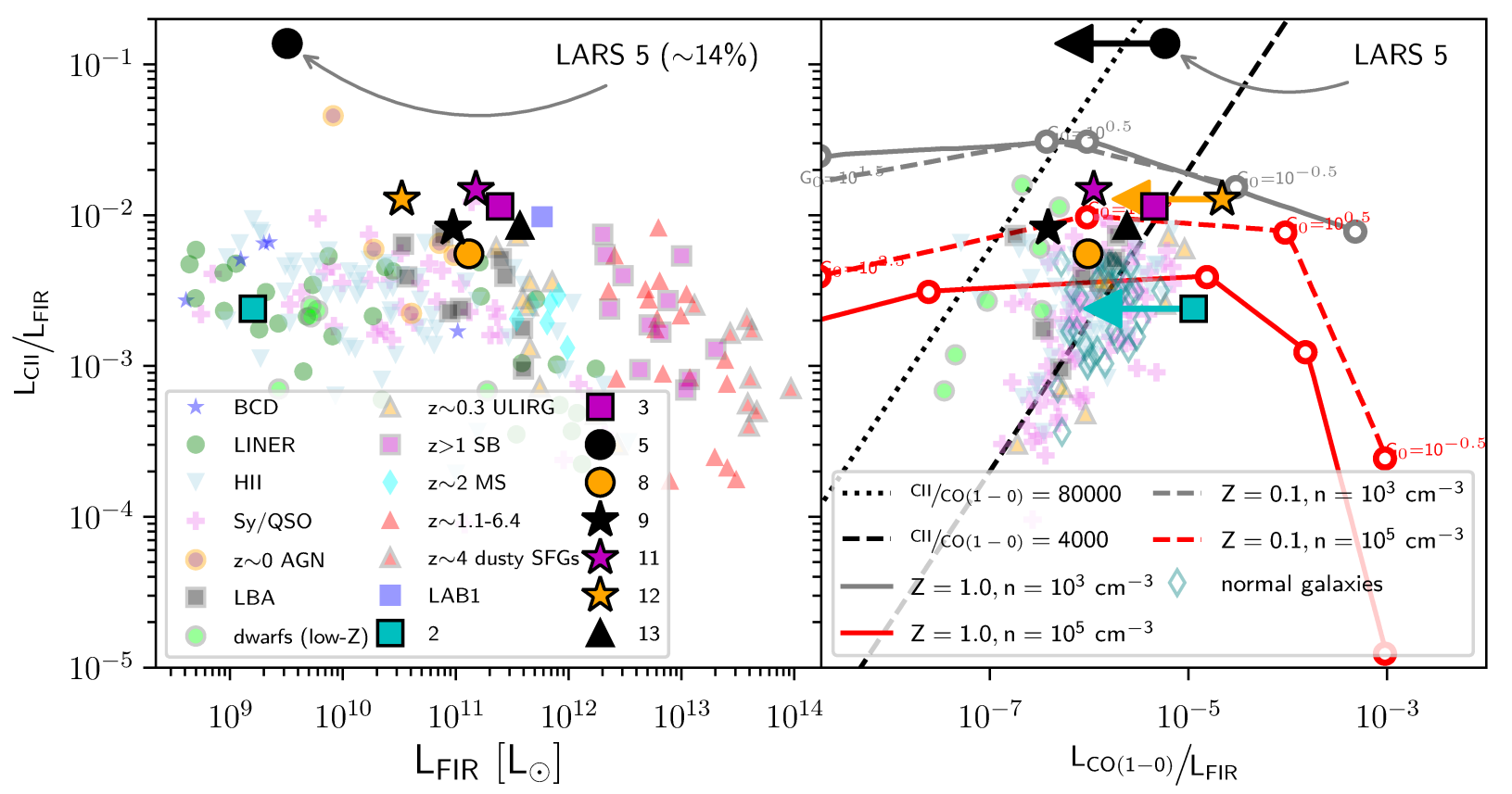

Fig. 12. $L_{\mathrm{CII}} 158 \mu \mathrm{m}$ line to $L_{\mathrm{FIR}_{n}, \mathrm{H} 88}$ ratio for LARS galaxies as a function of $L_{\mathrm{FIR}}$ (left panel) and as a function of the $L_{\mathrm{CO} 10}$ line to $L_{\mathrm{FIR}}, \mathrm{H} 88$ ratio (right panel), together with reference data. For the left plot, we updated the compilation in Graciá-Carpio et al. (2011, Fig. 1), and added samples of Lyman break analogues (LBA) from Contursi et al. (2017), local low-metallicity dwarfs from Cormier et al. (2014, 2015), $z \sim 0$ active galactic nuclei from the CARS survey (Smirnova-Pinchukova et al. 2019), $z \sim 0.3$ ULIRGs from Magdis et al. (2014), $z>1$ starbursts (SB $>1$ ) taken from Carilli \& Walter (2013), $z \sim 2$ main-sequence galaxies (Zanella et al. 2018), the Lyman alpha Blob 1 (LAB1) at $z \sim 3.1$ from Umehata et al. (2017), and $z>4$ dusty star-forming galaxies (Bothwell et al. 2017). The right hand plot is based on Contursi et al. (2017, Fig. 9), which we updated by adding normal galaxies from De Breuck et al. (2011) (originally drawn from Stacey et al. 2010), as well as local low-metallicity dwarfs from Cormier et al. $(2010,2014,2015)$ and Madden \& Cormier (2018). Black dotted and dashed lines in the right panel indicate constant [C II]-to-CO(1-0) ratios of 80000 and 4000 respectively. Red and grey solid curves show PDR models from Kaufman et al. (1999) for densities of $n=10^{5}$ and $n=10^{3} \mathrm{~cm}^{-3}$ at solar metallicity $(Z=1.0)$, while dashed curves indicate low metallicity $(Z=0.1)$. For the low-metallicity cases, the changing FUV radiation field along the curves is given in steps of 1 dex in $G_{0}$ from right to left.

as the fraction of gas mass with densities higher than $10^{4.5} \mathrm{~cm}^{-3}$. The results for LARS 3 and 8 are summarized in Table 14, and the $\chi^{2}$ solution planes are shown in Fig. D.1.

\section{Discussion}

\subsection{Turbulence-driven Lyman alpha escape}

As shown in Table 11, those LARS galaxies with the longest gas depletion times (Group 3: LARS 1, 2, 4, 7) have (on average) the largest total gas fractions (while having the lowest molecular gas fractions) and the lowest energy densities $\left(U_{\min }\right)$, compared to the other two groups of LARS galaxies. These galaxies really stand out in that they have very high Ly $\alpha$ escape fractions, while their scattering distances (a measure of the path length a Ly $\alpha$ photon travels before it escapes) are short. The combined properties of being gas-rich and having short scattering distances, is evidence of a kinematic-driven escape in which Ly $\alpha$ photons are shifted out of resonance relatively close to their origin. Previous high-resolution HST imaging in the optical, $\mathrm{H} \alpha$, and UV further reveal a sufficiently clumpy ISM (Messa et al. 2019) and discs without substantial bulge in those LARS galaxies. Moreover, Herenz et al. (2016) studied the H $\alpha$ kinematics of LARS galaxies and revealed that the highest Ly $\alpha$ escape fractions are found in dispersion-dominated systems. Hence, we are likely witnessing early stages of disc formation, similar to the theoretical predictions of Dekel et al. (2009) for high- $z$ galaxies. In such a scenario, cold gas accretion primarily causes disc instabilities that lead to the formation of massive clumps due to shear (each containing a few percent of the disc mass). Encounters between the clumps in the disc further stir up velocity dispersion, which at the same time enables Ly $\alpha$ photons to escape. The timescale of this process, however, is similar to the timescale of turbulent dissipation, so that the dynamical friction between the massive 
Table 12. Far infrared continuum and line luminosities.

\begin{tabular}{|c|c|c|c|c|c|c|c|c|}
\hline ID & $\begin{array}{r}L_{\mathrm{TIR}} \\
3-1100 \mu \mathrm{m} \\
{\left[10^{42} \mathrm{erg} \mathrm{s}^{-1}\right]} \\
\end{array}$ & $\begin{array}{r}L_{\mathrm{FIR}_{\mathrm{W}}} \\
40-500 \mu \mathrm{m} \\
{\left[10^{42} \mathrm{erg} \mathrm{s}^{-1}\right]} \\
\end{array}$ & $\begin{array}{r}L_{\mathrm{FIR}_{n, \mathrm{SED}}} \\
40-120 \mu \mathrm{m} \\
{\left[10^{42} \mathrm{erg} \mathrm{s}^{-1}\right]} \\
\end{array}$ & $\begin{array}{r}L_{\mathrm{FIR}_{n, \mathrm{H} 88}} \\
40-120 \mu \mathrm{m} \\
{\left[10^{42} \mathrm{erg} \mathrm{s}^{-1}\right]} \\
\end{array}$ & $\begin{array}{r}L_{\mathrm{CII}} \\
{\left[10^{39} \mathrm{erg} \mathrm{s}^{-1}\right]}\end{array}$ & $\begin{array}{r}L_{\mathrm{OI}_{63}} \\
{\left[10^{39} \mathrm{erg} \mathrm{s}^{-1}\right]}\end{array}$ & $\begin{array}{r}L_{\mathrm{OIII}_{88}} \\
{\left[10^{39} \mathrm{erg} \mathrm{s}^{-1}\right]}\end{array}$ & $\begin{array}{r}L_{\mathrm{CO} 10} \\
{\left[10^{36} \mathrm{erg} \mathrm{s}^{-1}\right]}\end{array}$ \\
\hline 1 & $54.81_{-14.4}^{42.48}$ & $31.43_{-6.79}^{28.54}$ & $24.6_{-4.03}^{16.81}$ & $19.64_{-0.04}^{14.89}$ & - & - & - & $<48$ \\
\hline 2 & $\begin{array}{l}7.27_{-2.66}^{5.73} \\
\end{array}$ & $6.14_{-2.2}^{4.62}$ & $3.86_{-1.41}^{3.39}$ & $7.08_{-3.09}^{0.01}$ & $17 \pm 8$ & $62 \pm 43$ & - & $<80$ \\
\hline 3 & $1690.33_{-61.83}^{449.23}$ & $950.04_{-15.56}^{135.73}$ & $796.7_{-0.08}^{147.86}$ & $136.79_{-102.34}^{287.82}$ & $1564 \pm 36$ & $2994 \pm 145$ & $1918 \pm 124$ & $619 \pm 31$ \\
\hline 4 & $102.71_{-4.48}^{12.08}$ & $58.66_{-2.2}^{6.25}$ & $47.34_{-3.84}^{3.49}$ & $27.22_{-0.22}^{21.18}$ & - & - & - & - \\
\hline 5 & $20.88_{-4.32}^{10.39}$ & $12.35_{-1.01}^{3.28}$ & $10.44_{-0.69}^{2.75}$ & $9.15_{-0.01}^{7.07}$ & $1260 \pm 223$ & - & $671 \pm 114$ & $<53$ \\
\hline 6 & $6.91_{-1.67}^{4.64}$ & $4.85_{-0.44}^{1.65}$ & $3.65_{-0.12}^{1.17}$ & $5.15_{-0.02}^{4.06}$ & - & - & - & - \\
\hline 7 & $76.85_{-3.93}^{19.1}$ & $43.81_{-3.55}^{12.16}$ & $34.61_{-4.94}^{7.06}$ & $20.62_{-0.04}^{15.66}$ & - & - & - & $<62$ \\
\hline 8 & $745.8_{-164.1}^{238}$ & $502.59_{-81.5}^{171.45}$ & $369.91_{-64.07}^{145.05}$ & $645.18_{-2.25}^{0.5}$ & $3580 \pm 549$ & - & - & $629 \pm 14$ \\
\hline 9 & $650.46_{-91.46}^{10.24}$ & $362.57_{-25.97}^{19.57}$ & $291.66_{-38.07}^{3.35}$ & $178.83_{-0.58}^{0.55}$ & $1471 \pm 29$ & $1284 \pm 119$ & - & $69 \pm 7$ \\
\hline 10 & $171.95_{-57.06}^{23.8}$ & $139.8_{-47.5}^{16.97}$ & $100.63_{-54.53}^{24.54}$ & $83.99_{-0.14}^{0.01}$ & - & - & - & $<434$ \\
\hline 11 & $996.71_{-153.26}^{373.73}$ & $579.55_{-73.8}^{247.91}$ & $428.6_{-21.22}^{195.41}$ & $603.07_{-0.06}^{1.56}$ & $8930 \pm 542$ & - & - & $671 \pm 43$ \\
\hline 12 & $256.77_{-3.14}^{22.65}$ & $127.87_{-5.96}^{20.97}$ & $106.62_{-6.55}^{20.6}$ & $29.93_{-21.21}^{60.7}$ & $383 \pm 55$ & $532 \pm 158$ & - & $<653$ \\
\hline 13 & $2742.85_{-258.7}^{163.12}$ & $1424.78_{-55.27}^{39.71}$ & $1225.98_{-72.1}^{8.15}$ & $208.94_{-1.05}^{146.47}$ & $1785 \pm 182$ & $2124 \pm 446$ & - & $502 \pm 45$ \\
\hline 14 & $148.8_{-3.65}^{25.16}$ & $59.7_{-7.83}^{21.27}$ & $51.61_{-1.9}^{10.75}$ & $99.89_{-0.03}^{76.77}$ & - & - & - & - \\
\hline
\end{tabular}

Notes. For continuum (TIR and FIR) luminosities we integrate the best-fit Draine et al. (2007) SED model for each model, using a variety of ranges along the wavelength axis. For total infrared (TIR), we use 3-1100 $\mu \mathrm{m}$, as defined in Kennicutt \& Evans (2012). In order to compare our measurements to previous studies (as done in Fig. 12), we further calculate several versions of the FIR luminosity: $L_{\mathrm{FIR}}$, which is integrated over $40-500 \mu \mathrm{m}$ and used on the $x$-axis of Fig. 12, and $L_{\mathrm{FIR}_{\mathrm{n}} \mathrm{H} 88}$, which is the FIR luminosity valid for the range of $40-120 \mu \mathrm{m}$, but estimated from single flux densities at 60 and $100 \mu \mathrm{m}$ as defined by Helou et al. (1988). The luminosity $L_{\mathrm{FIR}_{\mathrm{n} H 88}}$ is used for the [C II]-to-FIR ratio that is given on the $y$-axis in Fig. 12. For comparison, we also provide $L_{\mathrm{FIR}, \mathrm{SED}}$, which is the SED-integrated luminosity along the same range of $40-120 \mu \mathrm{m}$. The line luminosities are derived via Gaussian fitting.

Table 13. Results from PDR models of Kaufman et al. (1999, 2006).

\begin{tabular}{lccc}
\hline \hline ID & $\chi^{2}$ & $\begin{array}{c}n \\
{\left[\mathrm{~cm}^{-3}\right]}\end{array}$ & $G_{0}$ \\
\hline 2 & 0.05 & 5620 & 562 \\
12 & 0.4 & 1780 & 562 \\
13 & 0.1 & 562 & 1000 \\
\hline
\end{tabular}

Notes. The solution space $\left(n, G_{0}, \chi^{2}\right)$ is shown in Fig. C.1.

clumps finally leads to loss of angular momentum and migration to the centre where a bulge is formed. We thus conclude that this process significantly enhances $\operatorname{Ly} \alpha$ escape on timescales of a few hundred megayears. This scenario is also in agreement with simulations of molecular gas (at high redshift) by Kimm et al. (2019), who find that Ly $\alpha$ scatterings and escape are already significant on cloud-scales.

\subsection{Stellar-feedback-driven Lyman alpha escape}

Other LARS galaxies (Group 1: 3, 5, 12,13) have much shorter gas depletion times. They will run out of fuel in less than $\sim 1 \mathrm{Gyr}$. Also, their physical properties are distinct. They have the highest energy density $\left(U_{\min }\right)$ and longest Ly $\alpha$ scattering distances. From HST imaging we also recognize that these galaxies show a rather compact and more centralized light distribution. This suggests that the bulk of the gas and star formation is concentrated in a bulge-like structure, explaining higher average energy densities. This is also supported by our molecular line radiative transfer modelling of LARS 3 (member of Group 1), which indicates a very high mean molecular gas density of $\sim 10^{4} \mathrm{~cm}^{-3}$. On top of that we observed $\mathrm{CO}(3-2) / \mathrm{CO}(1-0) \sim 1$ in LARS 13 (also a member of Group 1), which is consistent with highly excited gas that is thermalized even up to the $\mathrm{CO} J=3$ level.

Our observations thus imply strong stellar feedback where Ly $\alpha$ photons may escape through channels in the ISM. Although the overall low gas content supports final escape, the photons need to travel significantly larger distances until they reach the point of last scattering. In this scenario the dust content thus plays an important role for $\operatorname{Ly} \alpha$ to escape.

\subsection{Robustness of our results against GDR prescriptions}

We test whether the high gas fractions and long total gas depletion times seen in Group 3 of LARS galaxies might be caused by the adopted power law in the gas-to-dust ratio versus metallicity prescription. For that purpose, we calculate depletion times and gas fractions using a constant GDR of 500. Given the fact that GDR $=500$ is slightly higher than the average GDR found from the aforementioned metallicity-dependent prescription, we find an average increase in the gas fraction of $5 \%$ for the whole sample. Roughly the same increase of $5 \%$ is seen for the three groups of LARS galaxies. This is not surprising given the fact that none of the groups are strongly biased towards lower or higher metallicities compared to the other groups. Thus, a constant GDR has no effect on the relative change of gas fractions or total gas depletion times amongst the groups of LARS galaxies, and our results cannot be caused by an over-prediction of the dependence between GDR and metallicity.

\section{Conclusion and summary}

The Lyman alpha reference sample contains $14 z \sim 0$ starforming galaxies that have continuum sizes, stellar masses, and 


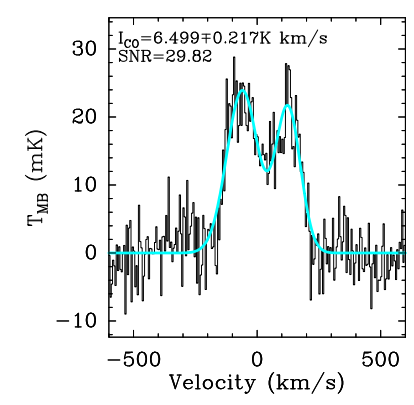

(1) LARS 08 CO (3-2)

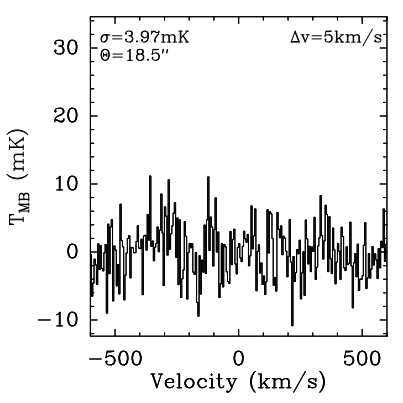

(2) residual

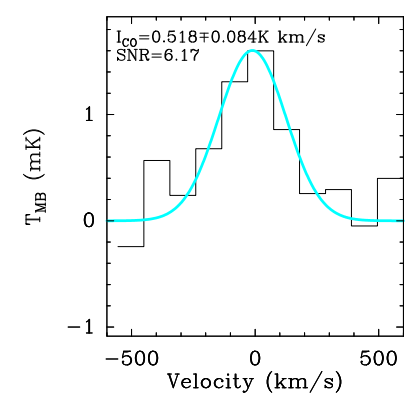

(3) LARS 13 CO (3-2)

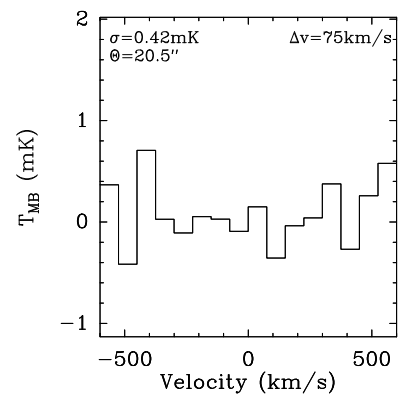

(4) residual

Fig. 13. Detections of CO(3-2) with APEX/SHeFI for LARS 8 (Col. 1) and 13 (Col. 3). Spectra are shown on the $T_{\mathrm{MB}}$ scale in milli Kelvin. Single or double Gaussian fits were performed to calculate the line flux. They are shown as cyan solid lines. The residuals after subtraction of the fits from the data are shown in panels (2) and (4).

Table 14. Interpretation of the molecular line ratios observed in LARS 3 and 8 .

\begin{tabular}{cccccc}
\hline \hline ID & $\begin{array}{c}\langle n\rangle \\
{\left[\mathrm{cm}^{-3}\right]}\end{array}$ & $\begin{array}{c}T \\
{[\mathrm{~K}]}\end{array}$ & $\begin{array}{c}\text { Width } \\
{[\mathrm{dex}]}\end{array}$ & $\begin{array}{c}f_{\text {dense }} \\
{[\%]}\end{array}$ & $\chi^{2}$ \\
\hline 3 & $\sim 8000$ & 30 & 0.6 & 36 & 1.5 \\
8 & $\sim 80$ & 25 & 0.6 & $<1$ & 5.1 \\
\hline
\end{tabular}

Notes. Assuming a log-normal distribution of densities with a fixed width of $0.6 \mathrm{dex}$ and a fixed temperature (using results from the dust models), we derive the mass-weighted mean density $\langle n\rangle$, as well as the corresponding dense gas fraction $f_{\text {dense }}$.

rest-frame absolute magnitudes similar to $2<z<3$ star-forming galaxies and massive Lyman alpha emitters (Guaita et al. 2015). In this paper, we present observations of LARS galaxies obtained with Herschel/PACS, SOFIA/FIFI-LS, the IRAM $30 \mathrm{~m}$ telescope, and APEX, targeting far-infrared continuum and emission lines of [C II $] 158 \mu \mathrm{m}$, [O I] $63 \mu \mathrm{m}$, and [O III $] 88 \mu \mathrm{m}$, as well as low- $J$ CO lines. In combination with archival farinfrared data (WISE, AKARI, and IRAS), we applied the models of Draine \& Li (2007) and derived parameters such as dust mass, average energy density, and PDR mass, using a Bayesian approach. Total gas masses were calculated for all LARS galaxies in a homogeneous way using a metallicity-dependent GDR, allowing us to establish the KS relation for all 14 LARS galaxies (see Fig. 9).

For those eight galaxies with a detection in [C II] $158 \mu \mathrm{m}$, we compared the relative [C II] $158 \mu \mathrm{m}$ line strength to the total farinfrared luminosity (see Fig. 12). For five LARS galaxies - with a detection of at least two emission lines, either fine-structure or molecular - a basic PDR analysis was performed, enabling us to estimate the average PDR gas density and FUV radiation field strength (see Fig. C.1).

We have further applied novel radiative transfers models ${ }^{9}$ (Puschnig et al., in prep.), taking into account that molecular emission lines emerge from a multi-density medium (with a lognorm density distribution) rather than from a single density gas. Using multi- $J \mathrm{CO}, \mathrm{HCN}(1-0)$, and $\mathrm{HCO}^{+}(1-0)$ observations of LARS 3 and 8 enabled us to derive mean mass-weighted molecular gas densities of these two galaxies. From the analysis of our data we conclude the following:

LARS covers a wide dynamic range in the derived properties, with FIR-based star formation rates from $\sim 0.5-100 M_{\odot} \mathrm{yr}^{-1}$, gas

\footnotetext{
9 http://wWw . densegastoolbox.com
}

fractions between $\sim 15-80 \%$ and gas depletion times ranging from a few hundred Myr up to more than $10 \mathrm{Gyr}$.

The distribution of LARS galaxies in the $\Sigma_{\text {gas }}$ versus $\Sigma_{\text {SFR }}$ is quite heterogeneous. However, after defining three groups of galaxies according to their gas depletion times, we observe that the group (LARS 1, 2, 4, 7) with the longest gas depletion times, relatively high gas surface densities $\left(\Sigma_{\text {gas }}\right)$, and low star formation rate densities $\left(\Sigma_{\mathrm{SFR}}\right)$, has (by far) the highest Ly $\alpha$ escape fraction. A relatively strong approximately linear trend is found between the Ly $\alpha$ escape fraction and total gas depletion time (see Fig. 11). We argue that the $\operatorname{Ly} \alpha$ escape in those galaxies is driven by accretion-induced turbulence in the star-forming gas that shifts the Ly $\alpha$ photons out of resonance close to the places where they originate (see Fig. 14). This scenario is supported by several other findings. From previously published optical, $\mathrm{H} \alpha$, and UV imaging, we recognize that these galaxies are very clumpy and most importantly do not show any form of strong bulge, in agreement with our finding of a low average energy density. We speculate that the clumps are the result of recent or ongoing cold gas accretion, which (1) triggered the clump formation and (2) injected turbulence that has not yet dissipated, and thus facilitates Ly $\alpha$ escape.

Another grouping of LARS galaxies is found in the KS plot. They have relatively low gas surface densities $\Sigma_{\text {gas }}$ that are more similar to observations in normal spirals or main sequence galaxies. However, their extreme star formation rate densities suggest very high star formation efficiencies (LARS 3, 5, 12, 13), corresponding to gas depletion times of a few $100 \mathrm{Myr}$ only. We argue that the Ly $\alpha$ escape in those galaxies (which is on the order of a few percent only) is facilitated by an environment that is radiation dominated and highly ionized. This is supported by our observations of very high average energy densities $\left(U_{\min }\right)$. Hubble Space Telescope imaging of these galaxies further shows a compact, centralized light distribution in those galaxies. In such an environment Ly $\alpha$ most likely escapes through channels that are a product of strong stellar feedback. This scenario is also supported by high- $J$ CO observations of LARS 13 that suggest highly excited gas that is thermalized at least up to the $J=3$ level. Further evidence is found from molecular gas radiative transfer modelling of LARS 3 that suggests very high mean molecular gas densities of $\sim 10^{4} \mathrm{~cm}^{-3}$. However, it seems that this scenario is less efficient in driving up Ly $\alpha$ escape fractions (compared to the previously described turbulence scenario found in other LARS galaxies), because the photons still undergo scatterings farther out in the halo (suggested by the longer scattering distances) and are thus more prone to dust absorption. 

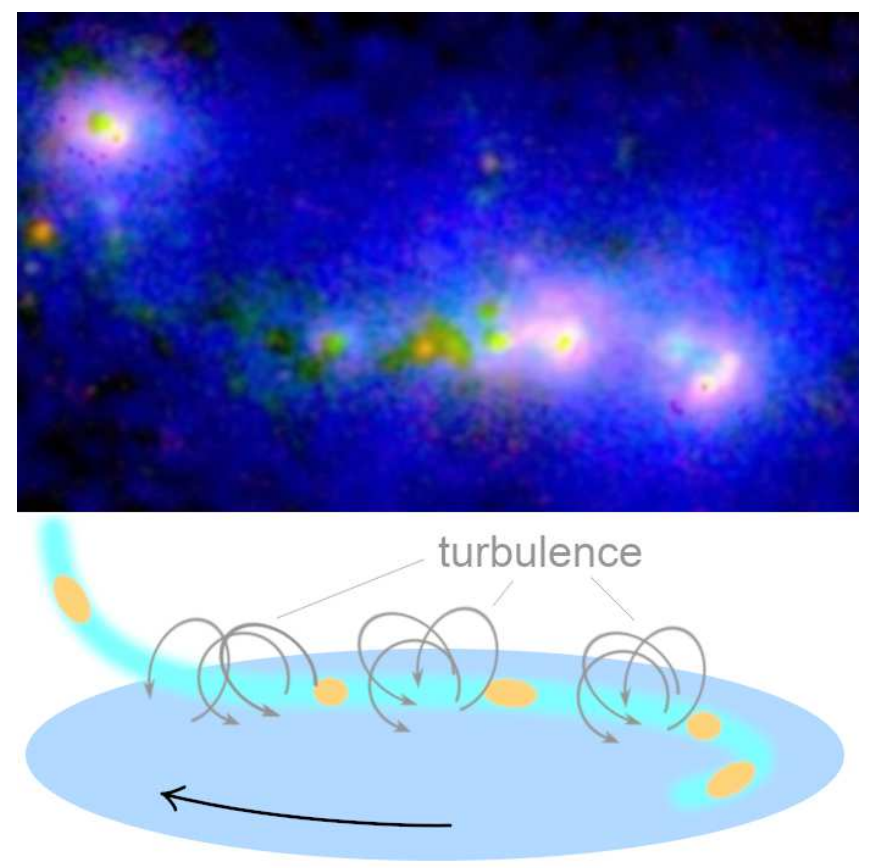

Fig. 14. Top: colour image of LARS 2 with red, green, and blue channels encoding $\mathrm{H} \alpha$, UV continuum, and $\mathrm{Ly} \alpha$ (scaled to show details). Bottom: sketch of turbulence-driven enhanced Ly $\alpha$ escape during disc formation.

We further report on an extreme [C II] $158 \mu \mathrm{m}$ excess in LARS 5, the highest [C II]-to-FIR ratio observed in a non-active galactic nucleus galaxy to date. LARS 5 is known to have an extreme stellar-driven outflow of gas. We find that the extreme [C II] $158 \mu \mathrm{m}$ line strength (corresponding to $\sim 14 \pm 3 \%$ of the FIR) must be related to the outflow as well. This is supported by the fact that the [C II $] 158 \mu \mathrm{m}$ line is blue shifted compared to the systemic velocity.

Acknowledgements. JP acknowledges funding from the European Research Council (ERC) under the European Union's Horizon 2020 research and innovation programme (grant agreement No.726384/Empire). JP owes special thanks to all IRAM Granada staff. In particular JP thanks Claudia Marka for numerous fruitful discussions, help with the heterodyne receiver setup, and data reduction; Nicolas Billot for assigning additional telescope time when observing time was lost due to scheduling constraints or weather; Israel Hermelo for helping to create OTF scripts; Sandra Trevino for support during the first heterodyne pool week in September 2014; and Carsten Kramer for discussions about our observing strategy and absolute calibration accuracy. This work has benefited from research funding from the European Community's Seventh Framework Programme, which covered JP's travelling expenses for IRAM 30m observations connected to programmes 082-14 and 064-15. JP thanks all APEX staff in Chile, in particular Karl Torstensson for giving great support by throwing light on the new features of the data reduction environment GILDAS/CLASS. GILDAS is a collection of state-of-the-art software oriented toward (sub-)millimetre radio astronomical applications (either single-dish or interferometer). JP thanks Jérôme Pety and Sébastien Bardeau for help with GILDAS/CLASS related questions. M.H acknowledges the support of the Swedish Research Council, Vetenskapsrådet and the Swedish National Space Board (SNSB), and is a Fellow of the Knut and Alice Wallenberg Foundation. D.K. is supported by the Centre National d'Études Spatiales (CNES)/Centre National de la Recherche Scientifique (CNRS); convention no 131425 . This work also benefits from data obtained with the Herschel spacecraft, which was designed, built, tested, and launched under a contract to ESA managed by the Herschel/Planck Project team by an industrial consortium under the overall responsibility of the prime contractor Thales Alenia Space (Cannes), and including Astrium (Friedrichshafen) responsible for the payload module and for system testing at spacecraft level, Thales Alenia Space (Turin) responsible for the service module, and Astrium (Toulouse) responsible for the telescope, with in excess of a hundred subcontractors. Based on observations made with the NASA/DLR Stratospheric Observatory for Infrared Astronomy
(SOFIA). SOFIA is jointly operated by the Universities Space Research Association, Inc. (USRA), under NASA contract NNA17BF53C, and the Deutsches SOFIA Institut (DSI) under DLR contract 50 OK 0901 to the University of Stuttgart. This publication makes use of data products from the Wide-field Infrared Survey Explorer, which is a joint project of the University of California, Los Angeles, and the Jet Propulsion Laboratory/California Institute of Technology, funded by the National Aeronautics and Space Administration. This research is based on observations with AKARI, a JAXA project with the participation of ESA. This research has made use of the NASA/IPAC Infrared Science Archive, which is operated by the Jet Propulsion Laboratory, California Institute of Technology, under contract with the National Aeronautics and Space Administration. Funding for the Sloan Digital Sky Survey (SDSS) has been provided by the Alfred P. Sloan Foundation, the Participating Institutions, the National Aeronautics and Space Administration, the National Science Foundation, the US Department of Energy, the Japanese Monbukagakusho, and the Max Planck Society. The SDSS website is http://www.sdss.org/. The SDSS is managed by the Astrophysical Research Consortium (ARC) for the Participating Institutions. The Participating Institutions are The University of Chicago, Fermilab, the Institute for Advanced Study, the Japan Participation Group, Johns Hopkins University, Los Alamos National Laboratory, the Max-Planck-Institute for Astronomy (MPIA), the Max-Planck-Institute for Astrophysics (MPA), New Mexico State University, University of Pittsburgh, Princeton University, the United States Naval Observatory, and the University of Washington. This research made use of Montage, funded by the National Aeronautics and Space Administration's Earth Science Technology Office, Computational Technologies Project, under Cooperative Agreement Number NCC5-626 between NASA and the California Institute of Technology. The code is maintained by the NASA/IPAC Infrared Science Archive.

\section{References}

Accurso, G., Saintonge, A., Catinella, B., et al. 2017, MNRAS, 470, 4750 Adams, J. J., Uson, J. M., Hill, G. J., \& MacQueen, P. J. 2011, ApJ, 728, 107

Ahn, S.-H., Lee, H.-W., \& Lee, H. M. 2001, ApJ, 554, 604

Ahn, S.-H., Lee, H.-W., \& Lee, H. M. 2002, ApJ, 567, 922

Amorín, R., Muñoz-Tuñón, C., Aguerri, J. A. L., \& Planesas, P. 2016, A\&A, 588, A23

Appleton, P. N., Guillard, P., Boulanger, F., et al. 2013, ApJ, 777, 66

Appleton, P. N., Diaz-Santos, T., Fadda, D., et al. 2018, ApJ, 869, 61

Belitsky, V., Lapkin, I., Monje, R., et al. 2006, in Society of Photo-Optical Instrumentation Engineers (SPIE) Conference Series, Proc. SPIE, 6275, $62750 \mathrm{G}$

Bendo, G. J., Wilson, C. D., Warren, B. E., et al. 2010, MNRAS, 402, 1409

Bertin, E., \& Arnouts, S. 1996, A\&AS, 117, 393

Bigiel, F., Leroy, A., Walter, F., et al. 2008, AJ, 136, 2846

Bigiel, F., Leroy, A. K., Walter, F., et al. 2011, ApJ, 730, L13

Bigiel, F., Leroy, A. K., Jiménez-Donaire, M. J., et al. 2016, ApJ, 822, L26

Bolatto, A. D., Leroy, A. K., Jameson, K., et al. 2011, ApJ, 741, 12

Bolatto, A. D., Wolfire, M., \& Leroy, A. K. 2013, ARA\&A, 51, 207

Bolatto, A. D., Wong, T., Utomo, D., et al. 2017, ApJ, 846, 159

Bothwell, M. S., Aguirre, J. E., Aravena, M., et al. 2017, MNRAS, 466, 2825

Bridge, J. S., Hayes, M., Melinder, J., et al. 2018, ApJ, 852, 9

Brinchmann, J., Charlot, S., White, S. D. M., et al. 2004, MNRAS, 351, 1151

Calzetti, D., Wilson, G. W., Draine, B. T., et al. 2018, ApJ, 852, 106

Cannon, J. M., McClure-Griffiths, N. M., Skillman, E. D., \& Côté, S. 2004, ApJ,

607,274

Carilli, C. L., \& Walter, F. 2013, ARA\&A, 51, 105

Catinella, B., Schiminovich, D., Kauffmann, G., et al. 2012, A\&A, 544, A65

Chabrier, G. 2003, PASP, 115, 763

Colombo, D., Kalinova, V., Utomo, D., et al. 2018, MNRAS, 475, 1791

Contursi, A., Baker, A. J., Berta, S., et al. 2017, A\&A, 606, A86

Cormier, D., Madden, S. C., Hony, S., et al. 2010, A\&A, 518, L57

Cormier, D., Madden, S. C., Lebouteiller, V., et al. 2014, A\&A, 564, A121

Cormier, D., Madden, S. C., Lebouteiller, V., et al. 2015, A\&A, 578, A53

Cortese, L., Bekki, K., Boselli, A., et al. 2016, MNRAS, 459, 3574

Cowie, L. L., \& Hu, E. M. 1998, AJ, 115, 1319

Cowie, L. L., Barger, A. J., \& Hu, E. M. 2011, ApJ, 738, 136

Daddi, E., Dickinson, M., Morrison, G., et al. 2007, ApJ, 670, 156

Davis, T. A., Alatalo, K., Bureau, M., et al. 2013, MNRAS, 429, 534

De Breuck, C., Maiolino, R., Caselli, P., et al. 2011, A\&A, 530, L8

De Looze, I., Cormier, D., Lebouteiller, V., et al. 2014, A\&A, 568, A62

Dekel, A., Sari, R., \& Ceverino, D. 2009, ApJ, 703, 785

Dijkstra, M., Haiman, Z., \& Spaans, M. 2006a, ApJ, 649, 14

Dijkstra, M., Haiman, Z., \& Spaans, M. 2006b, ApJ, 649, 37

Doi, Y., Takita, S., Ootsubo, T., et al. 2015, PASJ, 67, 50

Draine, B. T., \& Li, A. 2007, ApJ, 657, 810

Draine, B. T., Dale, D. A., Bendo, G., et al. 2007, ApJ, 663, 866 
Duval, F., Schaerer, D., Östlin, G., \& Laursen, P. 2014, A\&A, 562, A52 Duval, F., Östlin, G., Hayes, M., et al. 2016, A\&A, 587, A77

Elbaz, D., Daddi, E., Le Borgne, D., et al. 2007, A\&A, 468, 33 Engelbracht, C. W., Rieke, G. H., Gordon, K. D., et al. 2008, ApJ, 678, 804 Evans, N. J., II, Dunham, M. M., Jørgensen, J. K., et al. 2009, ApJS, 181, 32 Evans, N. J., II, Heiderman, A., \& Vutisalchavakul, N. 2014, ApJ, 782, 114 Fruchter, A. S., \& Hook, R. N. 2002, PASP, 114, 144

Galametz, M., Madden, S. C., Galliano, F., et al. 2011, A\&A, 532, A56 Galliano, F. 2018, MNRAS, 476, 1445

Galliano, F., Madden, S. C., Jones, A. P., et al. 2003, A\&A, 407, 159

Galliano, F., Madden, S. C., Jones, A. P., Wilson, C. D., \& Bernard, J.-P. 2005 A\&A, 434, 867

Galliano, F., Dwek, E., \& Chanial, P. 2008, ApJ, 672, 214

Galliano, F., Hony, S., Bernard, J.-P., et al. 2011, A\&A, 536, A88

Gao, Y., \& Solomon, P. M. 2004a, ApJS, 152, 63

Gao, Y., \& Solomon, P. M. 2004b, ApJ, 606, 271

Genzel, R., Tacconi, L. J., Gracia-Carpio, J., et al. 2010, MNRAS, 407, 2091

Genzel, R., Tacconi, L. J., Combes, F., et al. 2012, ApJ, 746, 69

Genzel, R., Tacconi, L. J., Lutz, D., et al. 2015, ApJ, 800, 20

Graciá-Carpio, J., Sturm, E., Hailey-Dunsheath, S., et al. 2011, ApJ, 728, L7

Guaita, L., Melinder, J., Hayes, M., et al. 2015, A\&A, 576, A51

Güsten, R., Nyman, L. Å., Schilke, P., et al. 2006, A\&A, 454, L13

Gutermuth, R. A., Pipher, J. L., Megeath, S. T., et al. 2011, ApJ, 739, 84

Habing, H. J. 1968, Bull. Astron. Inst. Neth., 19, 421

Hakobyan, A. A., Adibekyan, V. Z., Aramyan, L. S., et al. 2012, A\&A, 544, A81

Hao, C.-N., Kennicutt, R. C., Johnson, B. D., et al. 2011, ApJ, 741, 124

Hayes, M. 2015, PASA, 32, e027

Hayes, M., Östlin, G., Schaerer, D., et al. 2010, Nature, 464, 562

Hayes, M., Östlin, G., Schaerer, D., et al. 2013, ApJ, 765, L27

Hayes, M., Östlin, G., Duval, F., et al. 2014, ApJ, 782, 6

Heiderman, A., Evans, N. J., II, Allen, L. E., Huard, T., \& Heyer, M. 2010, ApJ, 723, 1019

Helou, G., Khan, I. R., Malek, L., \& Boehmer, L. 1988, ApJS, 68, 151

Herenz, E. C., Gruyters, P., Orlitova, I., et al. 2016, A\&A, 587, A78

Herenz, E. C., Urrutia, T., Wisotzki, L., et al. 2017, A\&A, 606, A12

Herrera-Camus, R., Sturm, E., Graciá-Carpio, J., et al. 2018a, ApJ, 861, 94

Herrera-Camus, R., Sturm, E., Graciá-Carpio, J., et al. 2018b, ApJ, 861, 95

Hirashita, H., Tajiri, Y. Y., \& Kamaya, H. 2002, A\&A, 388, 439

Hubble, E. P. 1926, ApJ, 64, 321

Hunt, L., Bianchi, S., \& Maiolino, R. 2005, A\&A, 434, 849

Hunt, L. K., García-Burillo, S., Casasola, V., et al. 2015, A\&A, 583, A114

James, A., Dunne, L., Eales, S., \& Edmunds, M. G. 2002, MNRAS, 335, 753

Jameson, K. E., Bolatto, A. D., Leroy, A. K., et al. 2016, ApJ, 825, 12

Jaskot, A. E., \& Oey, M. S. 2014, ApJ, 791, L19

Jiménez-Donaire, M. J., Bigiel, F., Leroy, A. K., et al. 2017, MNRAS, 466, 49

Jiménez-Donaire, M. J., Bigiel, F., Leroy, A. K., et al. 2019, ApJ, 880, 127

Juneau, S., Narayanan, D. T., Moustakas, J., et al. 2009, ApJ, 707, 1217

Kahre, L., Walterbos, R. A., Kim, H., et al. 2018, ApJ, 855, 133

Kaufman, M. J., Wolfire, M. G., Hollenbach, D. J., \& Luhman, M. L. 1999, ApJ, 527,795

Kaufman, M. J., Wolfire, M. G., \& Hollenbach, D. J. 2006, ApJ, 644, 283

Kawada, M., Baba, H., Barthel, P. D., et al. 2007, PASJ, 59, S389

Kennicutt, R. C., Jr. 1998, ApJ, 498, 541

Kennicutt, R. C., \& Evans, N. J. 2012, ARA\&A, 50, 531

Kimm, T., Blaizot, J., Garel, T., et al. 2019, MNRAS, 486, 2215

Kroupa, P., \& Weidner, C. 2003, ApJ, 598, 1076

Kruijssen, J. M. D., Schruba, A., Chevance, M., et al. 2019, Nature, 569, 519

Krumholz, M. R., Dekel, A., \& McKee, C. F. 2012, ApJ, 745, 69

Kunth, D., Mas-Hesse, J. M., Terlevich, E., et al. 1998, A\&A, 334, 11

Lada, C. J., Lombardi, M., \& Alves, J. F. 2010, ApJ, 724, 687

Lada, C. J., Forbrich, J., Lombardi, M., \& Alves, J. F. 2012, ApJ, 745, 190

Lang, D. 2014, AJ, 147, 108

Leroy, A. K., Walter, F., Brinks, E., et al. 2008, AJ, 136, 2782

Leroy, A. K., Bolatto, A., Gordon, K., et al. 2011, ApJ, 737, 12

Leroy, A. K., Walter, F., Sandstrom, K., et al. 2013, AJ, 146, 19

Leroy, A. K., Usero, A., Schruba, A., et al. 2017, ApJ, 835, 217

Lisenfeld, U., \& Ferrara, A. 1998, ApJ, 496, 145

Lisenfeld, U., Espada, D., Verdes-Montenegro, L., et al. 2011, A\&A, 534, A102

Lord, S. D. 1992, NASA Technical Memorandum 103957, Tech. Rep. (NASA)

Madden, S. C., \& Cormier, D. 2018, Proc. Int. Astron. Union, 14, 240

Magdis, G. E., Daddi, E., Elbaz, D., et al. 2011, ApJ, 740, L15

Magdis, G. E., Rigopoulou, D., Hopwood, R., et al. 2014, ApJ, 796, 63

Magrini, L., Bianchi, S., Corbelli, E., et al. 2011, A\&A, 535, A13

Mas-Hesse, J. M., Kunth, D., Tenorio-Tagle, G., et al. 2003, ApJ, 598, 858

Mathis, J. S., Mezger, P. G., \& Panagia, N. 1983, A\&A, 128, 212

Matthee, J., Sobral, D., Santos, S., et al. 2015, MNRAS, 451, 400

Meier, D. L., \& Terlevich, R. 1981, ApJ, 246, L109

Messa, M., Adamo, A., Â-stlin, G., et al. 2019, MNRAS, 487, 4238
Micheva, G., Östlin, G., Zackrisson, E., et al. 2018, A\&A, 615, A46

Muñoz-Mateos, J. C., Gil de Paz, A., Boissier, S., et al. 2009, ApJ, 701, 1965

Murakami, H., Baba, H., Barthel, P., et al. 2007, PASJ, 59, S369

Murphy, E. J., Condon, J. J., Schinnerer, E., et al. 2011, ApJ, 737, 67

Narayanan, D., Krumholz, M. R., Ostriker, E. C., \& Hernquist, L. 2012, MNRAS, 421, 3127

Neufeld, D. A. 1990, ApJ, 350, 216

Noeske, K. G., Faber, S. M., Weiner, B. J., et al. 2007, ApJ, 660, L47

Östlin, G., Hayes, M., Duval, F., et al. 2014, ApJ, 797, 11

Ott, S. 2010, in Astronomical Data Analysis Software and Systems XIX, eds. Y.

Mizumoto, K. I. Morita, \& M. Ohishi, ASP Conf. Ser., 434, 139

Ouchi, M., Shimasaku, K., Akiyama, M., et al. 2008, ApJS, 176, 301

Ouchi, M., Shimasaku, K., Furusawa, H., et al. 2010, ApJ, 723, 869

Pardy, S. A., Cannon, J. M., Östlin, G., et al. 2014, ApJ, 794, 101

Partridge, R. B., \& Peebles, P. J. E. 1967, ApJ, 147, 868

Peng, Y.-J., Lilly, S. J., Kovač, K., et al. 2010, ApJ, 721, 193

Pilbratt, G. L., Riedinger, J. R., Passvogel, T., et al. 2010, A\&A, 518, L1

Poglitsch, A., Waelkens, C., Geis, N., et al. 2010, A\&A, 518, L2

Popesso, P., Concas, A., Morselli, L., et al. 2019, MNRAS, 483, 3213

Pound, M. W., \& Wolfire, M. G. 2008, in Astronomical Data Analysis Software and Systems XVII, eds. R. W. Argyle, P. S. Bunclark, \& J. R. Lewis, ASP Conf. Ser., 394, 654

Puschnig, J. 2020, https://doi .org/10.5281/zenodo. 3686329

Puschnig, J., Hayes, M., Östlin, G., et al. 2017, MNRAS, 469, 3252

Rémy-Ruyer, A., Madden, S. C., Galliano, F., et al. 2014, A\&A, 563, A31

Rieke, G. H., Alonso-Herrero, A., Weiner, B. J., et al. 2009, ApJ, 692, 556

Rivera-Thorsen, T. E., Hayes, M., Östlin, G., et al. 2015, ApJ, 805, 14

Rodighiero, G., Daddi, E., Baronchelli, I., et al. 2011, ApJ, 739, L40

Roman-Duval, J., Gordon, K. D., Meixner, M., et al. 2014, ApJ, 797, 86

Runnholm, A., Hayes, M., Melinder, J., et al. 2020, ApJ, 892, 48

Saintonge, A., Kauffmann, G., Kramer, C., et al. 2011a, MNRAS, 415, 32

Saintonge, A., Kauffmann, G., Wang, J., et al. 2011b, MNRAS, 415, 61

Saintonge, A., Tacconi, L. J., Fabello, S., et al. 2012, ApJ, 758, 73

Salpeter, E. E. 1955, ApJ, 121, 161

Sandstrom, K. M., Leroy, A. K., Walter, F., et al. 2013, ApJ, 777, 5

Santos, S., Sobral, D., \& Matthee, J. 2016, MNRAS, 463, 1678

Scarlata, C., Colbert, J., Teplitz, H. I., et al. 2009, ApJ, 704, L98

Schaerer, D., \& Verhamme, A. 2008, A\&A, 480, 369

Schmidt, M. 1959, ApJ, 129, 243

Schruba, A., Leroy, A. K., Walter, F., et al. 2011, AJ, 142, 37

Schruba, A., Leroy, A. K., Walter, F., et al. 2012, AJ, 143, 138

Schruba, A., Leroy, A. K., Kruijssen, J. M. D., et al. 2017, ApJ, 835, 278

Scoville, N., Lee, N., Vanden Bout, P., et al. 2017, ApJ, 837, 150

Semenov, V. A., Kravtsov, A. V., \& Gnedin, N. Y. 2019, ApJ, 870, 79

Smirnova-Pinchukova, I., Husemann, B., Busch, G., et al. 2019, A\&A, 626, L3

Sobral, D., \& Matthee, J. 2019, A\&A, 623, A157

Sobral, D., Santos, S., Matthee, J., et al. 2018, MNRAS, 476, 4725

Solomon, P. M., \& Vanden Bout, P. A. 2005, ARA\&A, 43, 677

Stacey, G. J., Hailey-Dunsheath, S., Ferkinhoff, C., et al. 2010, ApJ, 724, 957

Stark, D. V., Kannappan, S. J., Wei, L. H., et al. 2013, ApJ, 769, 82

Tacconi, L. J., Neri, R., Genzel, R., et al. 2013, ApJ, 768, 74

Takita, S., Doi, Y., Ootsubo, T., et al. 2015, PASJ, 67, 51

Tenorio-Tagle, G., Silich, S. A., Kunth, D., Terlevich, E., \& Terlevich, R. 1999, MNRAS, 309, 332

Tomczak, A. R., Quadri, R. F., Tran, K.-V. H., et al. 2016, ApJ, 817, 118

Trainor, R. F., Strom, A. L., Steidel, C. C., et al. 2019, ApJ, 887, 85

Umehata, H., Matsuda, Y., Tamura, Y., et al. 2017, ApJ, 834, L16

Urrutia, T., Wisotzki, L., Kerutt, J., et al. 2019, A\&A, 624, A141

Utomo, D., Sun, J., Leroy, A. K., et al. 2018, ApJ, 861, L18

van der Tak, F. F. S., Black, J. H., Schöier, F. L., Jansen, D. J., \& van Dishoeck,

E. F. 2007, A\&A, 468, 627

Vassilev, V., Meledin, D., Lapkin, I., et al. 2008, A\&A, 490, 1157

Verhamme, A., Schaerer, D., \& Maselli, A. 2006, A\&A, 460, 397

Verhamme, A., Schaerer, D., Atek, H., \& Tapken, C. 2008, A\&A, 491, 89

Verhamme, A., Orlitová, I., Schaerer, D., \& Hayes, M. 2015, A\&A, 578, A7

Wall, J. V. 1996, QJRAS, 37, 519

Whitaker, K. E., van Dokkum, P. G., Brammer, G., \& Franx, M. 2012, ApJ, 754, L29

Whitaker, K. E., Franx, M., Leja, J., et al. 2014, ApJ, 795, 104

Wofford, A., Leitherer, C., \& Salzer, J. 2013, ApJ, 765, 118

Wong, T., \& Blitz, L. 2002, ApJ, 569, 157

Wright, E. L., Eisenhardt, P. R. M., Mainzer, A. K., et al. 2010, AJ, 140, 1868

Wu, J., Evans, N. J., II, Gao, Y., et al. 2005, ApJ, 635, L173

Wuyts, S., Förster Schreiber, N. M., van der Wel, A., et al. 2011, ApJ, 742, 96

Young, J. S., Xie, S., Tacconi, L., et al. 1995, ApJS, 98, 219

Zanella, A., Daddi, E., Magdis, G., et al. 2018, MNRAS, 481, 1976

Zhao, Y., Yan, L., \& Tsai, C.-W. 2016, ApJ, 824, 146

Zheng, Z.-Y., Wang, J., Rhoads, J., et al. 2017, ApJ, 842, L22 


\section{Appendix A: Bayesian joint posterior probability distribution for Draine and Li model parameters}
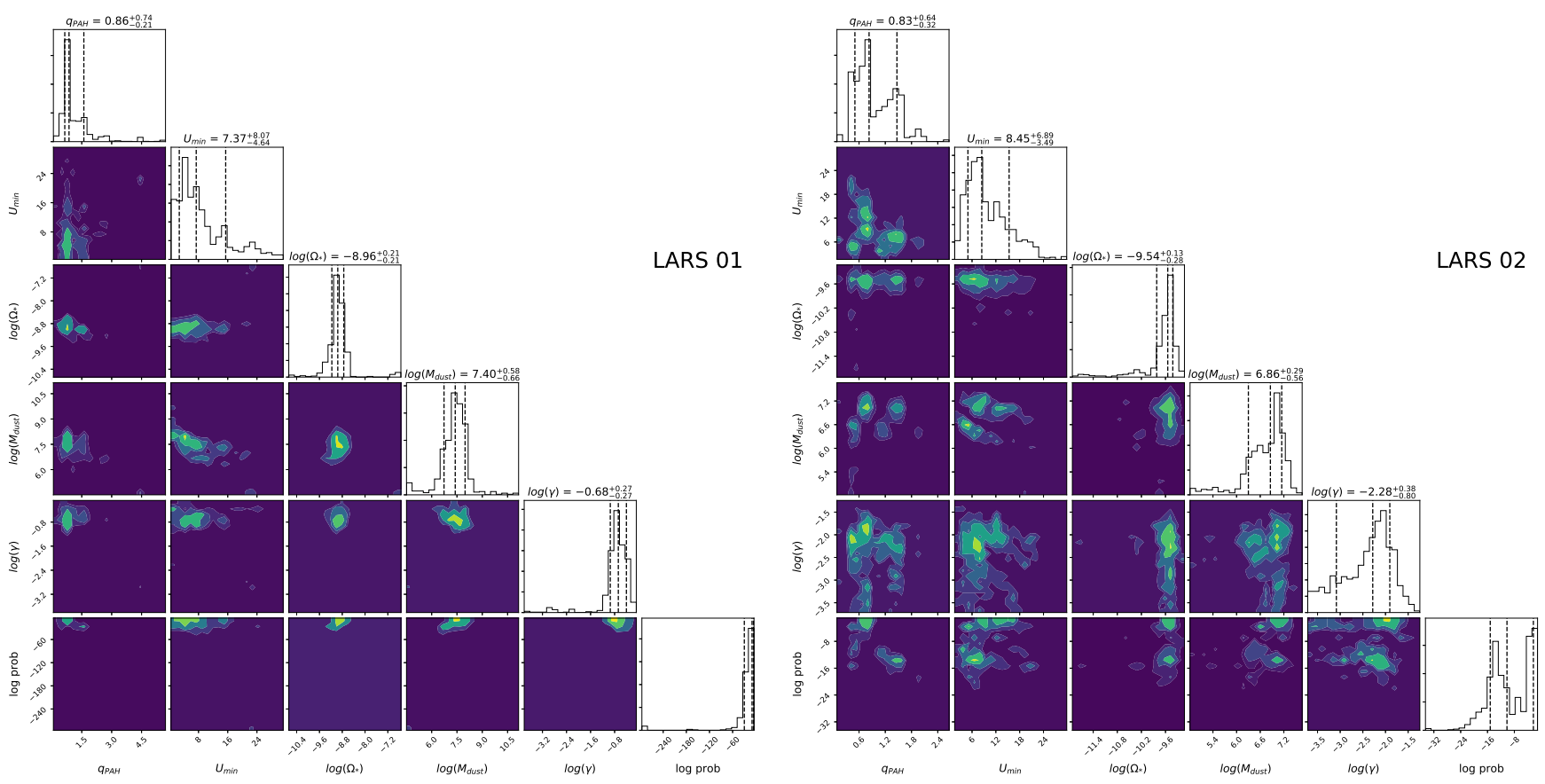

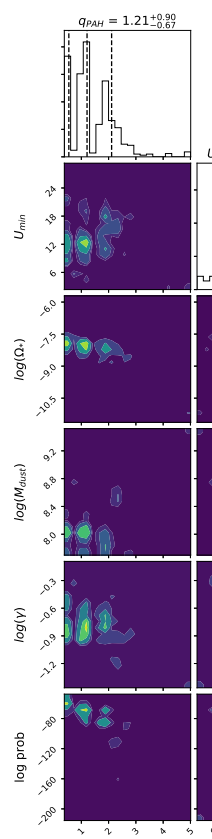

$q_{\text {PAH }}$

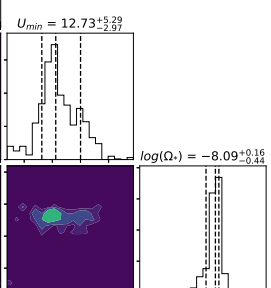

LARS 03

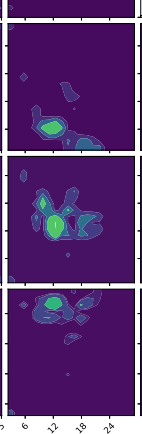

$u_{\min }$

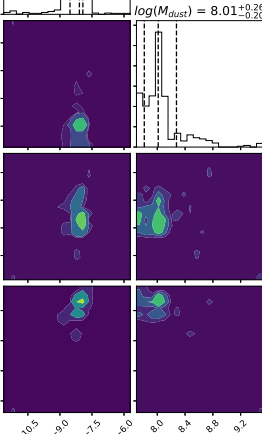

$\log (\Omega *$

$\log \left(M_{\text {duss }}\right)$

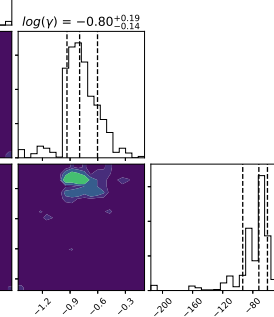

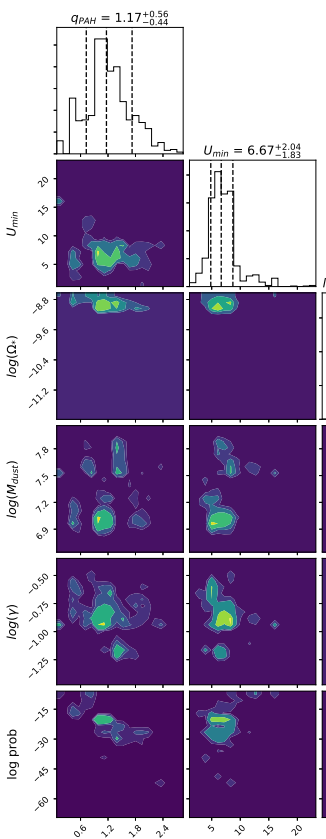

$q_{P A H}$

$u_{\min }$

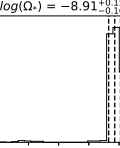

LARS 04

Fig. A.1. Corner plots showing the posterior probability distribution for the derived Draine and Li model parameters. 
A\&A 644, A10 (2020)
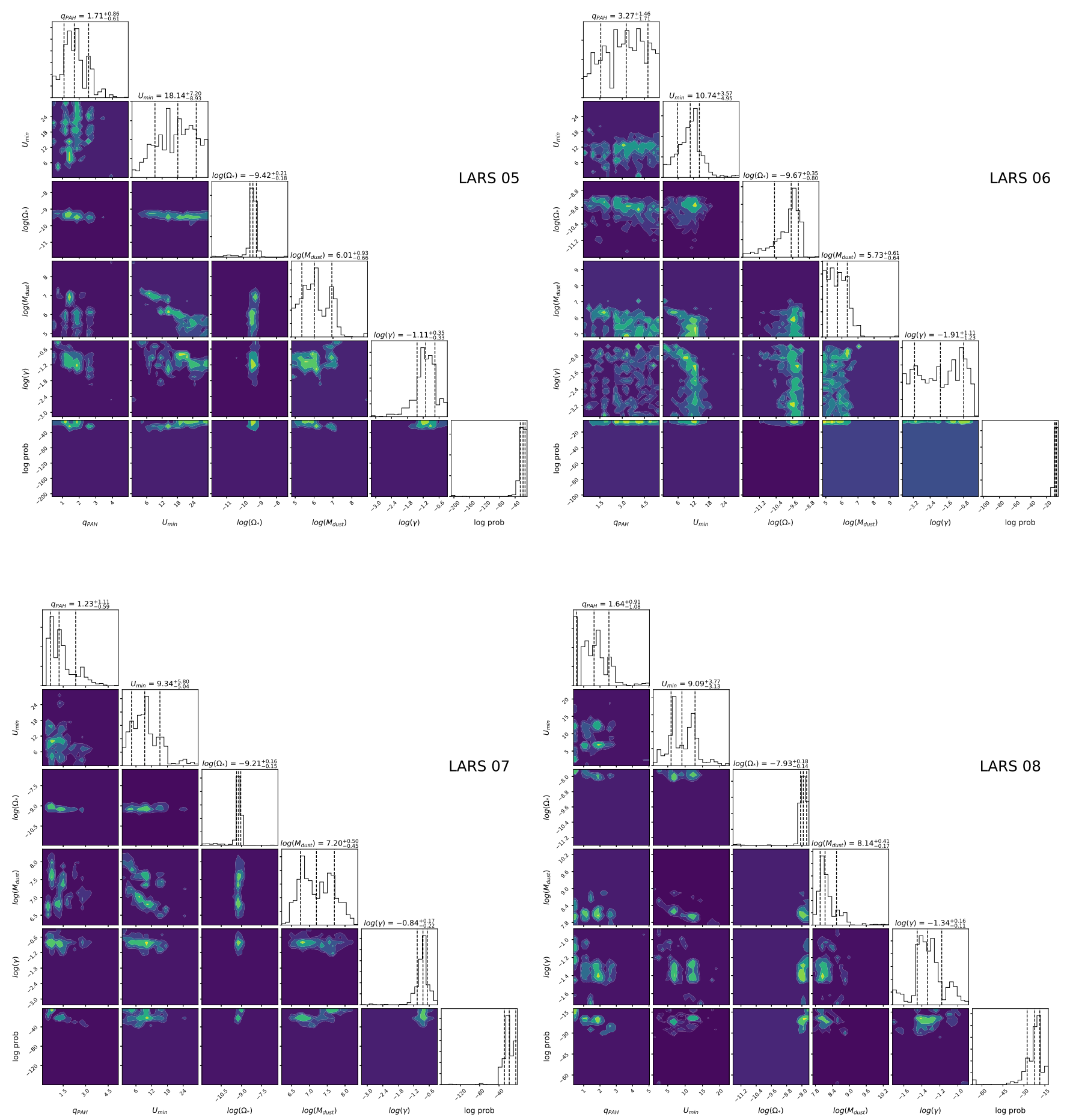

Fig. A.1. continued. 

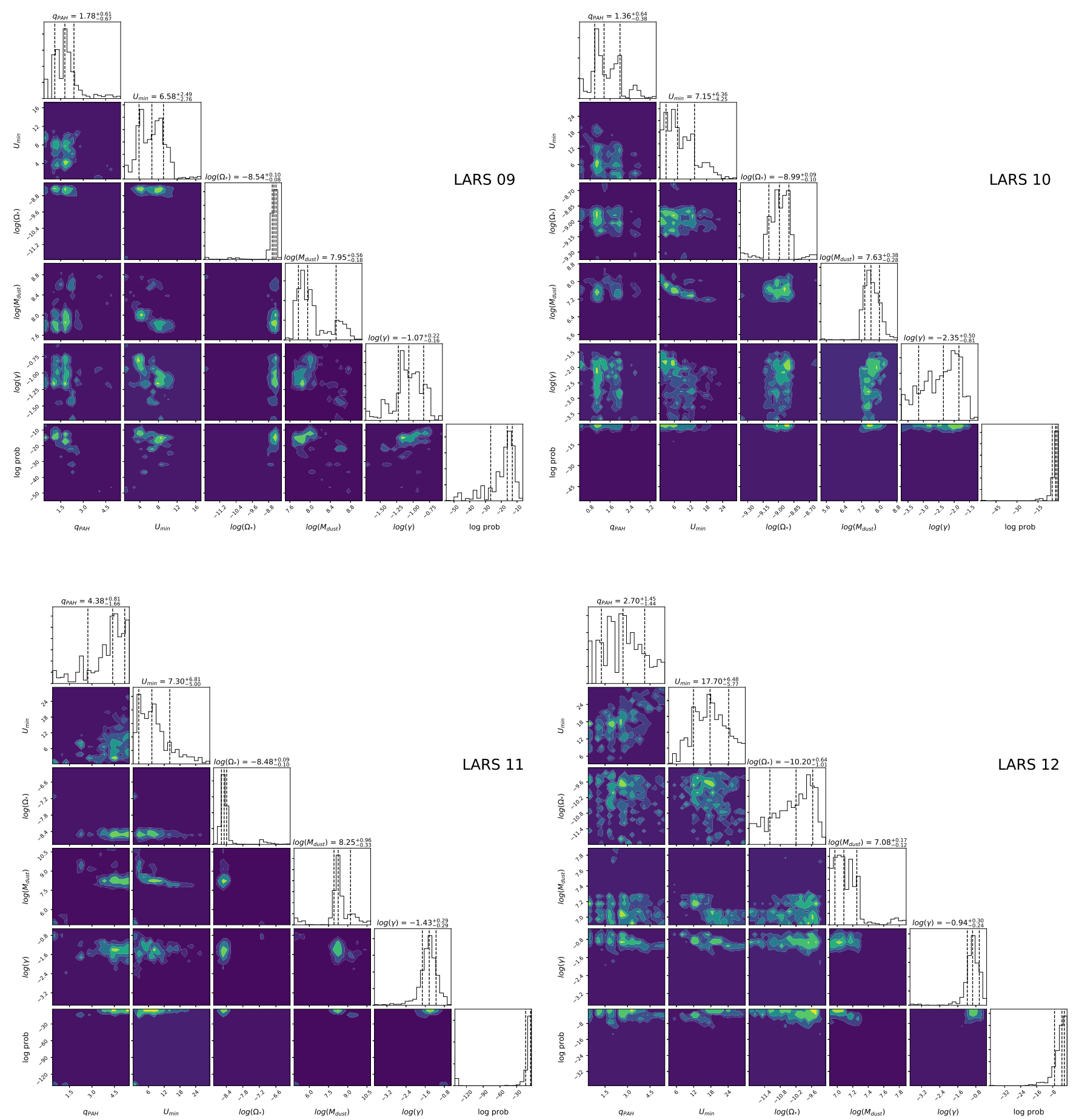

Fig. A.1. continued. 
A\&A 644, A10 (2020)
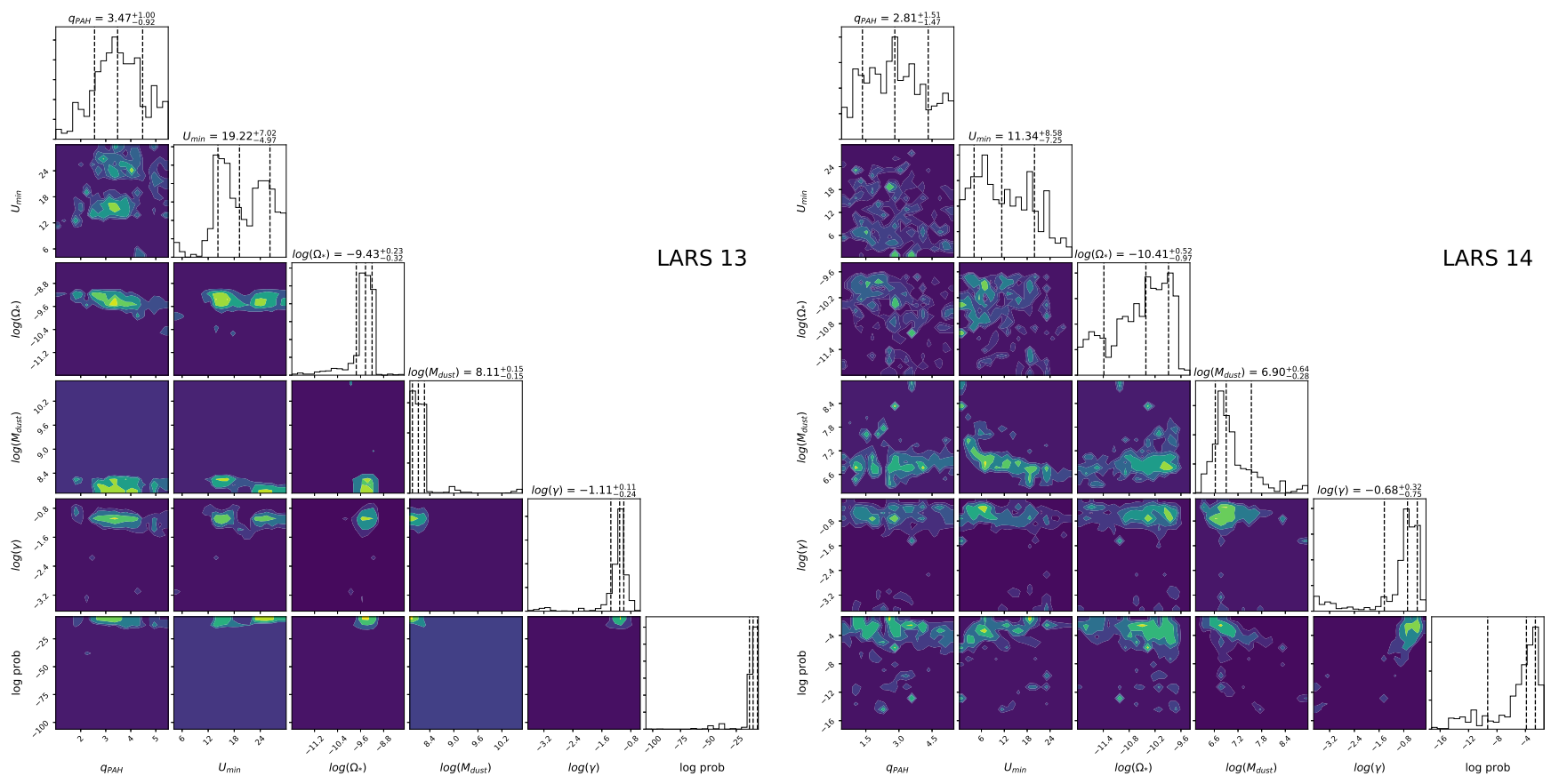

Fig. A.1. continued. 


\section{Appendix B: Draine and Li SED fits using the MCMC method}
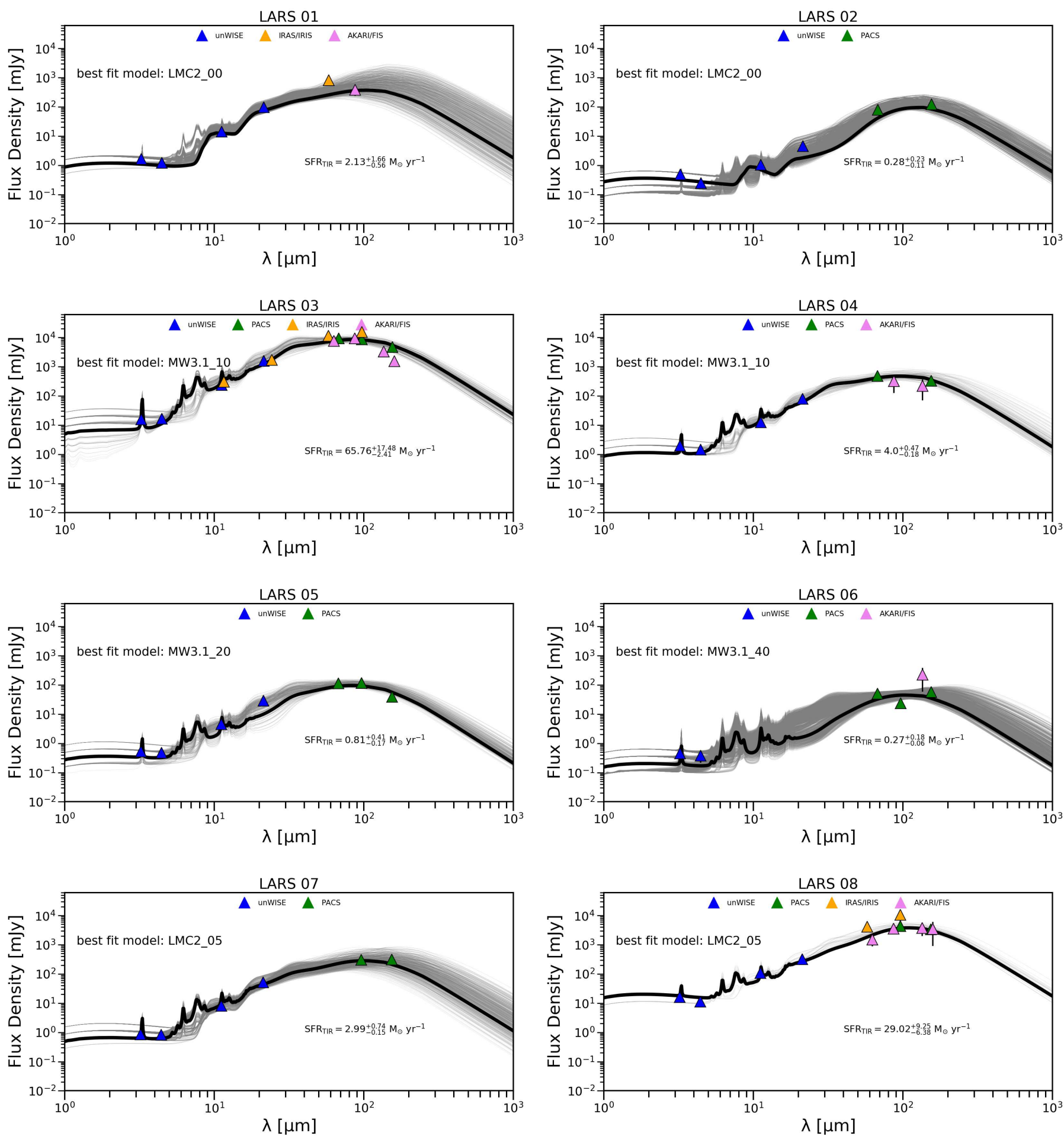

Fig. B.1. Infrared spectral energy distribution of LARS galaxies inferred from exploration of the parameter space using the Markov-chain Monte Carlo method. The best-fit SED is indicated by a thick black curve and was found from the $50 \%$ quantile of the logarithmic posterior distribution. The other fits that are shown (thin grey lines) are those lying within the quantile-based credible interval corresponding to $16 \%$ and $84 \%$. They thus represent our uncertainties. 

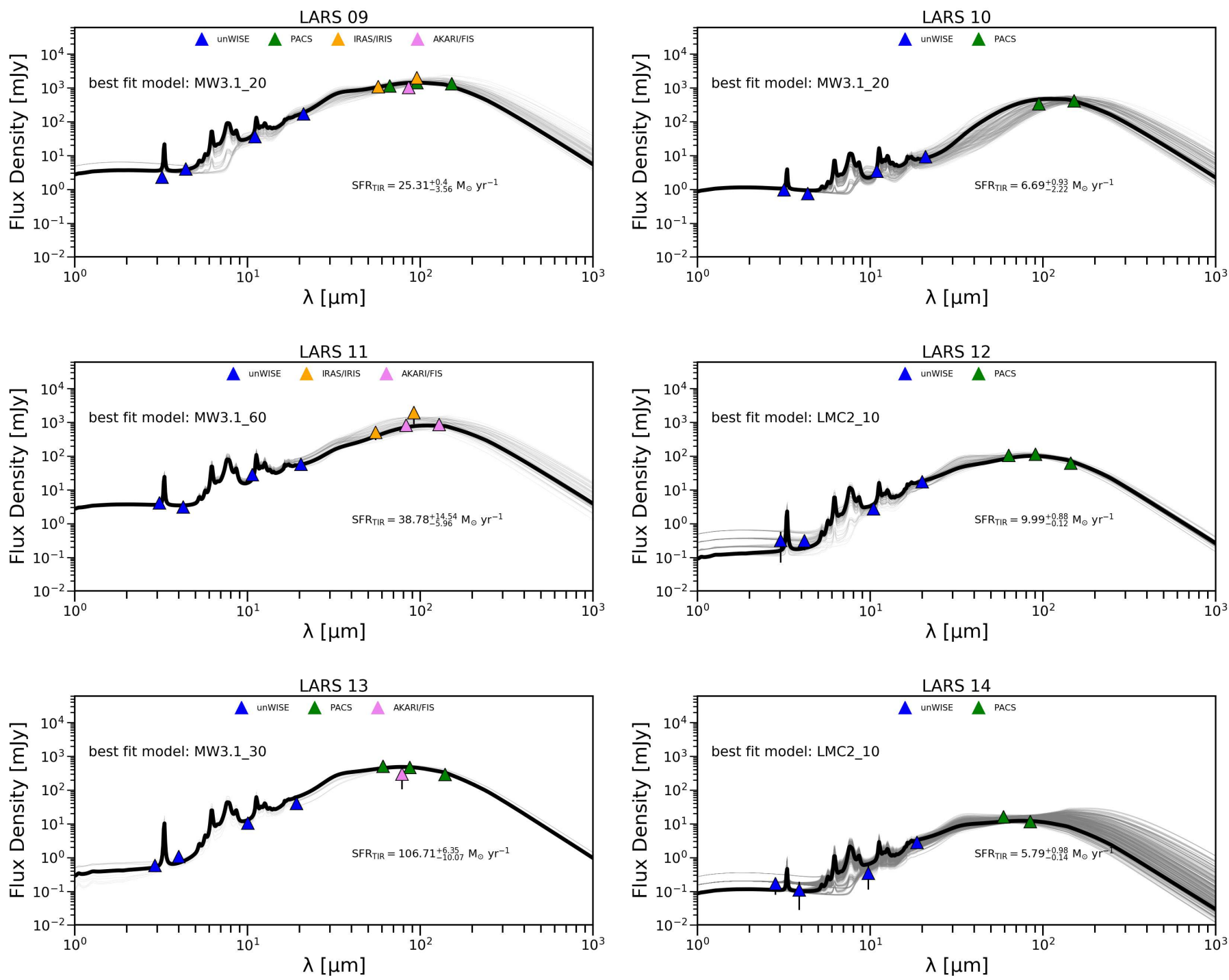

Fig. B.2. Same as in Fig. B.1.

\section{Appendix C: Fit quality of photon-dominated regions models}
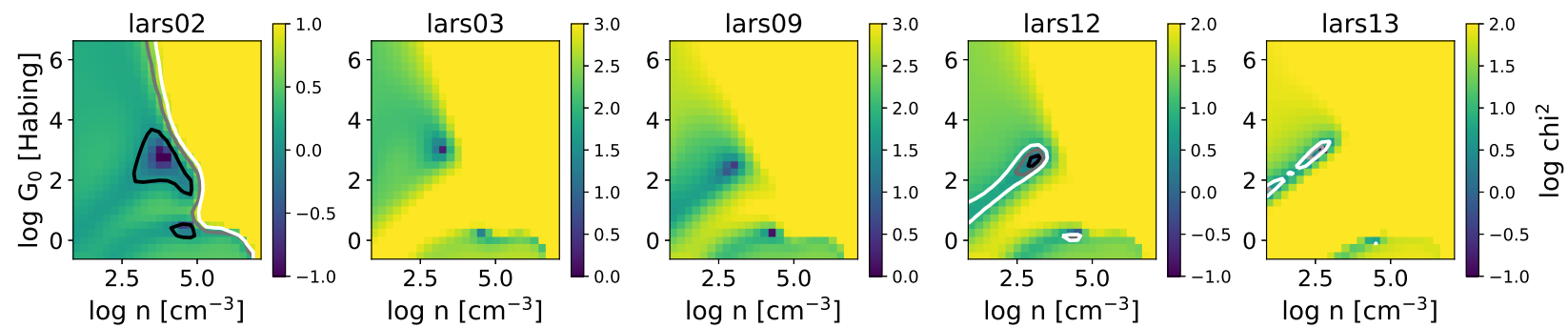

Fig. C.1. Logarithmic $\chi^{2}$ distribution of the PDR model across the $\left(n, G_{0}\right)$ solution plane. The contours show 1,2 , and $3 \sigma$ significance regions around the best model fits. We note that for LARS 3 and 9 (both merging galaxies) the models are not able to reproduce the observations. 


\section{Appendix D: Molecular gas density and temperature from line radiative transfer modelling}

Table D.1. Calculated molecular line ratios used as input for radiative transfer modelling of LARS 3 and 8.

\begin{tabular}{cccccc}
\hline \hline ID & Line & $\begin{array}{c}I_{\mathrm{mb}} \\
{\left[\mathrm{K} \mathrm{km} \mathrm{s}^{-1}\right]}\end{array}$ & $C_{\mathrm{AP}}$ & $\begin{array}{c}\theta \\
{[\operatorname{arcsec}]}\end{array}$ & Line/CO(1-0) \\
\hline 3 & $\mathrm{CO}(1-0)$ & 7.13 & 1.94 & 22.1 & 1.00 \\
3 & $\mathrm{CO}(2-1)$ & 15.93 & 3.58 & 11.0 & 1.02 \\
3 & $\mathrm{HCN}^{2}(1-0)$ & 1.06 & 1.00 & 28.7 & 0.13 \\
3 & $\mathrm{HCO}^{+}(1-0)$ & 1.08 & 1.00 & 28.6 & 0.13 \\
8 & $\mathrm{CO}(1-0)$ & 6.85 & 1.36 & 22.2 & 1.00 \\
8 & $\mathrm{CO}(2-1)$ & 11.70 & 1.10 & 11.1 & 0.35 \\
8 & $\mathrm{CO}(3-2)$ & 6.50 & 1.00 & 18.5 & 0.48 \\
8 & $\mathrm{HCN}(1-0)$ & 0.29 & 1.00 & 28.9 & 0.05 \\
8 & $\mathrm{HCO}^{+}(1-0)$ & 0.19 & 1.00 & 28.7 & 0.03 \\
\hline
\end{tabular}

Notes. The galaxy id is given in Col. 1, followed by the observed integrated main beam brightness temperature in Col. 2 and the aperture correction factor as well as the beam size in Cols. 3 and 4. Normalized line ratios (Col. 5) are then calculated as: $\left(I_{\mathrm{mb}} * C_{\mathrm{AP}} * \theta^{2}\right) /\left(I_{\mathrm{mb}, \mathrm{CO} 10} *\right.$ $\left.C_{\mathrm{AP}, \mathrm{CO} 10} * \theta_{\mathrm{CO} 10}^{2}\right)$. The squared beam size needs to be taken into account, as explained in Solomon \& Vanden Bout (2005).
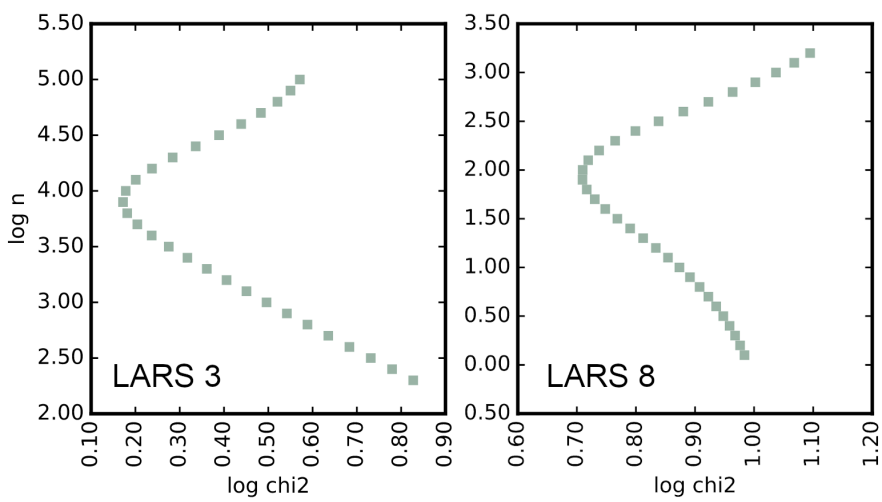

Fig. D.1. Mass-weighted mean molecular gas density vs. $\chi^{2}$ for LARS 3 and 8 . The (mass-weighted) mean density $(\log n)$ of the emitting gas could be derived using a novel radiative transfer approach. The models (Puschnig et al., in prep.) were minimized against observed line luminosities of $\mathrm{CO}(1-0), \mathrm{CO}(2-1), \mathrm{HCN}(1-0)$, and $\mathrm{HCO}^{+}(1-0)$ for LARS 3 and additional $\mathrm{CO}(3-2)$ for LARS 8.

\section{Appendix E: Choice of GDR and $\alpha_{\mathrm{CO}}$}

Given the fact that our galaxy sample is very heterogeneous in terms of metallicity, stellar mass, and star formation activity, we adopt a metallicity-dependent gas-to-dust ratio and $\mathrm{CO}-$ to- $\mathrm{H}_{2}$ conversion factor when calculating total and molecular gas masses respectively. For the galaxies with atomic gas mass estimates from H I $21 \mathrm{~cm}$ observations, we compare available recipes for GDR and $\alpha_{\mathrm{CO}}$. The results are shown in Table E.1. We
Table E.1. Mean deviation in dex of dust-based estimates of the molecular gas mass $\Delta M_{\mathrm{H}_{2}}$, i.e. dust-based total gas minus $M_{\mathrm{HI}}$ from Pardy et al. (2014) (see Table 1) and mean deviation in dex of dust-based estimates of the total gas mass $\Delta M_{\text {gas }}$, i.e. dust-based total gas minus $M_{\mathrm{HI}}+M_{\mathrm{H}_{2}}$.

\begin{tabular}{lcccccc}
\hline \hline & $\mathrm{N} 12$ & $\mathrm{~N} 12$ & $\mathrm{~S} 12$ & $\mathrm{~S} 12$ & $\mathrm{~A} 17$ & $\mathrm{~A} 17$ \\
& $\Delta M_{\mathrm{H}_{2}}$ & $\Delta M_{g}$ & $\Delta M_{\mathrm{H}_{2}}$ & $\Delta M_{g}$ & $\Delta M_{\mathrm{H}_{2}}$ & $\Delta M_{g}$ \\
\hline R14,Ref & 0.23 & 0.11 & 0.48 & 0.32 & 0.34 & 0.19 \\
R14,PL & 0.61 & 0.36 & 0.31 & 0.22 & 0.31 & 0.28 \\
R14,BPL & $\mathbf{0 . 2 3}$ & $\mathbf{0 . 1 1}$ & 0.48 & 0.32 & 0.34 & 0.19 \\
L11 & Neg & 0.14 & Neg & 0.52 & Neg & 0.14 \\
\hline
\end{tabular}

Notes. Deviations could only be calculated for galaxies with both $M_{\mathrm{HI}}$ and $M_{\mathrm{H}_{2}}$ measured (LARS 3, 8, 9, and 11), and are biased towards higher metallicities. We evaluate the deviations for a combination of prescriptions for the gas-to-dust ratio (R14: Rémy-Ruyer et al. 2014, L11: Leroy et al. 2011), and for the CO-to- $\mathrm{H}_{2}$ conversion factor (N12: Narayanan et al. 2012, S12: Schruba et al. 2012, A17: Accurso et al. 2017). Highlighted in bold are the model combinations that provide the best fits to our observations.

find that the combination of the prescriptions in Narayanan et al. (2012) (N12) for $\alpha_{\mathrm{CO}}$ and the linear GDR scaling in Rémy-Ruyer et al. (2014) give the best fit for our sample, with a deviation of only $\sim 0.1$ dex for the total gas mass (see Fig. 8). The sub-linear GDR scaling of Leroy et al. (2011) (L11) for example underestimates our observed molecular masses. The $\alpha_{\mathrm{CO}}$ prescriptions of Schruba et al. (2012) (S12) and Accurso et al. (2017) (A17) lead to deviations of $\sim 0.3 \mathrm{dex}$ and $\sim 0.2$ dex compared to our dustbased estimates. It is important to note that the prescriptions for $\alpha_{\mathrm{CO}}$ of N12, S12, and A17 are fundamentally different and rely on different assumptions. S12 assume a constant star formation efficiency or gas depletion time to derive their relation for the metallicity dependence of $\alpha_{\mathrm{CO}}$. A17 use combined $\mathrm{CO}(1-0)$ and [C II] $158 \mu \mathrm{m}$ observations and relate those to $\alpha_{\mathrm{CO}}$. In their prescription, $\alpha_{\mathrm{CO}}$ depends not only on metallicity, but also on the offset of the galaxy from the main sequence. The metallicity basically describes the total dust content available to shield $\mathrm{CO}$ from UV radiation and the offset from the main sequence describes the strength of this radiation field. On the other hand, N12 define $\alpha_{\mathrm{CO}}$ to depend on metallicity and CO surface brightness $I_{\mathrm{CO}}$ (i.e. velocity integrated brightness temperature). While the metallicity dependence in N12 has basically the same meaning as in S12 or A17, that is, it accounts for CO-dark gas at low dust contents, the dependence on $I_{\mathrm{CO}}$ makes $\alpha_{\mathrm{CO}}$ sensitive to environmental variations, such as variations in the density and temperature of the molecular gas. For example, it is known that ultra-luminous infrared galaxies (mergers) have lower $\alpha_{\mathrm{CO}}$ values, because in nuclear starbursts the molecular gas is denser and hotter with molecular gas being mostly non-virialized compared to normal star-forming regions. This leads to brighter $\mathrm{CO}$ emission (and thus lower $\alpha_{\mathrm{CO}}$ ) due to higher excitation temperatures. The fact that (1) the peak brightness temperature of the emission is sensitive to excitation temperature, and (2) the line width increases in the presence of non-virialized, dense, hot gas, makes $I_{\mathrm{CO}}$ sensitive to changing environmental conditions. Hence, the reason why the $\alpha_{\text {CO }}$ prescription of N12 best fits our observations is an indication for changing environmental conditions amongst LARS galaxies that have significant implications for $\alpha_{\mathrm{CO}}$. 